\title{
COLLOID THRUSTER TO TEACH ADVANCED ELECTRIC PROPULSION TECHNIQUES TO POST-SECONDARY STUDENTS
}

\author{
A Thesis \\ presented to \\ the Faculty of California Polytechnic State University, \\ San Luis Obispo
}

\author{
In Partial Fulfillment \\ of the Requirements for the Degree \\ Master of Science in Aerospace Engineering
}

by

Alexander M. Powaser

June 2019 
(c) 2019

Alexander M. Powaser

ALL RIGHTS RESERVED 


\title{
COMMITTEE MEMBERSHIP
}

\author{
TITLE: Colloid Thruster to Teach Advanced \\ Electric Propulsion Techniques to Post- \\ Secondary Students
}

AUTHOR: Alexander M. Powaser

DATE SUBMITTED: June 2019

COMMITTEE CHAIR: Amelia Greig, Ph.D.

Assistant Professor of Aerospace Engineering

COMMitTeE MeMBER: Kira Abercromby, Ph.D.

Associate Professor of Aerospace Engineering

COMmitTeE MEMBER: Karl Saunders, Ph.D.

Professor of Physics, Department Chair

COMMITTEE MEMBER: Joseph Vanherweg, MS

Design Engineer at Stellar Exploration 


\section{ABSTRACT \\ Colloid Thruster to Teach Advanced Electric Propulsion Techniques to Post-Secondary Students}

\section{Alexander M. Powaser}

Colloid thrusters, and electrospray thrusters as a whole, have been around since the 1960s. When they were first developed, the high efficiency and fine thrust control was overshadowed by the high power requirement for such a low thrust that the system provides. This caused the technology to be put on hold for aerospace applications. Now, as small satellites are becoming more prevalent, there has been a resurgence in interest in electrospray thruster technology. The recent advancements in technology allow electrospray thrusters to use significantly less power and occupy less volume than their predecessors. As electrospray technology continues to advance, these thrusters are meeting the demands of small satellite propulsion. As such, in an effort to keep the spacecraft propulsion curriculum current with today's technology, a colloid thruster is designed, built, tested, and implemented as a laboratory activity at California Polytechnic State University, San Luis Obispo.

Electrospray thrusters work by placing a voltage on an ionic liquid and extracting either beads of propellant or ions to generate thrust. By definition, colloid thrusters are a specific class of electrospray thrusters that use solvents, such as glycerol or formamide, to emit droplets or, in special cases, ions to generate thrust. To keep with the University's "Learn by Doing" pedagogical philosophy, the thruster for this activity is designed to have a tactile and experiential impact on the students. The final design is a scaled up configuration of an existing electrospray design so that the students can easily see each component with the naked eye and can be correlated to a real world thruster that they might see in industry.

As a laboratory experiment, the thruster needs to be able to utilize current equip- 
ment in the Space Environments and Testing Laboratory. One of the Student Vacuum Chambers (SVC) is utilized as well as two $1 \mathrm{kV}$ power supplies and a $100 \mathrm{~V}$ power supply. An indirect method of measuring performance metrics needs to be developed as there are no thrust balances sensitive enough in the lab designated for undergraduate use. As such, the students will be using the mass of the propellant, the time of operation, and knowledge of the propellant's properties to estimate the performance of the thruster.

To prove success of the thruster, a performance profile of the thruster is produced using an indirect method of measurement as well as visual observations of the thruster moving propellant byway of the electrospray theory. The tests show thrusts produced between 96-311 $\mu \mathrm{N}$ with an $\mathrm{I}_{\mathrm{sp}}$ ranging from 1270-1684 seconds. The visual evidence demonstrates propellant being collected as well as the operation of the thruster under the electrospray theory. The visual evidence also sheds light on which emission mode the thruster is operating at as well as a self-correcting failure mode that was occurring. The thruster is implemented as a lab for Cal Poly's AERO 402 Spacecraft Propulsion Lab in Fall 2018, and it receives positive feedback from the students through an anonymous survey.

While the colloid thruster demonstrates success in meeting performance and pedagogical goals, future work should be continued to improve the thruster. Further design and manufacturing work can be undertaken to improve the efficiency and decrease failure due to propellant impingement. Additionally, the procurement of power supplies capable of applying higher voltages can provide a greater range of operation which can enable a more dynamic student discovery of electrospray thrusters. 


\section{ACKNOWLEDGMENTS}

First, I would like to thank the Aerospace Engineering Department at Cal Poly for funding this thesis and allowing me to utilize their lab spaces. I want to thank Dr. Amelia Greig for being my advisor through this process, which was no easy task. In addition to being my advisor, she provided many opportunities for networking and collaborating with industry professionals and university researchers that have a great expertise in electrospray thrusters. Through her encouragement, I achieved more with this thesis than I thought possible.

Also at Cal Poly, I want to thank Cody Thompson. Through his "tribal knowledge" I was able to successfully manufacture this thruster. He also helped with all sorts of equipment procurement and CNC-machine some of the parts used in this project.

Last, but definitely not least, I want to thank my family for being a huge source of support and encouragement. While they didn't always understand my thesis, they were more than happy to listen and give word of encouragement or solidarity. My mom was even happy to proofread this thesis for grammar and spelling. I also want to give my extreme gratitude to my fiancée, Maddy Johnson. She has seen me through very late nights and extremely long days. She has been with me in the extreme highs and lowest of lows of this project. She, too, proofread this thesis many many times, and she was a great sounding board when I needed to figure out how to write something. I don't know how I could have done this without any of them.

I hope that this work helps continue the great legacy of the aerospace engineers that are produced at Cal Poly. May they learn and be impacted by the technologies and solutions that are invented and researched to continue humanity's presence in space. Maybe, by some chance, this lab will spark the idea to provide a solution for our current propulsion problems. Just in the event that any of the folks at NASA end 
up reading this, I hope that the idea sparked from this activity will solve human spaceflight propulsion problems, and I would be honored to fly it. Yes, I'm taking a play from Buzz Aldrin when he wrote his acknowledgements for his PhD dissertation. Let's see if Dr. Abercromby's theory is right! 


\section{TABLE OF CONTENTS}

Page

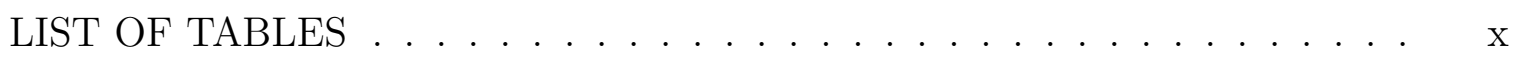

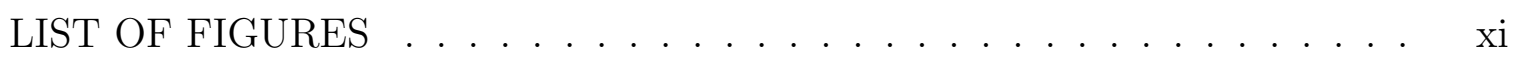

1 Introduction . . . . . . . . . . . . . . . . . 1

1.1 History and Application . . . . . . . . . . . . . 2

1.2 Electrospray Theory ........................... 3

1.3 Emitters ............................. 6

1.4 Taylor Cone . . . . . . . . . . . . . . . . . 7

1.5 Thesis Scope. . . . . . . . . . . . . . . . . . 9

2 Thruster Design . . . . . . . . . . . . . . . . . 11

2.1 Design Decisions . . . . . . . . . . . . . . . . 12

2.2 Analysis . . . . . . . . . . . . . . . . . . 15

2.3 Manufacturing . . . . . . . . . . . . . . . . 18

2.4 Limitations . . . . . . . . . . . . . . . . . . . . . 21

3 Methodology ... . . . . . . . . . . . . . . . . 23

3.1 Laboratory Equipment . . . . . . . . . . . . . . 23

3.2 Experimental Setup . . . . . . . . . . . . . . 25

3.3 Method of Indirect Performance Measurement . . . . . . . . . . . . 28

3.4 Method of Proving Electrospray Success . . . . . . . . . . . . . . 31

4 Results and Discussion . . . . . . . . . . . . . . . . 33

4.1 Visual Confirmation . . . . . . . . . . . . . . . 33

4.2 Estimated Performance Profile . . . . . . . . . . . . . . 37

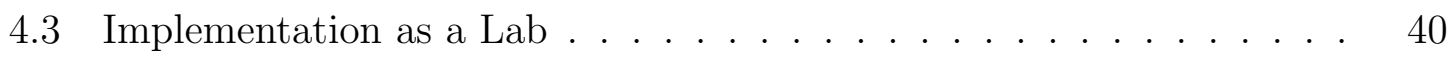


5 Conclusion . . . . . . . . . . . . . . . . . . . . 42

5.1 Future Work . . . . . . . . . . . . . . . . 44

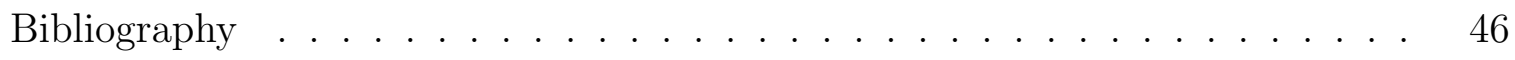

\section{APPENDICES}

A Colloid Thruster Part Drawings . . . . . . . . . . . . . 49

B Student Lab Document . . . . . . . . . . . . . . 56

C Instructor Lab Document . . . . . . . . . . . . . . . 64

D Operating Procedures .................... 74 


\section{LIST OF TABLES}

Table

Page

1 Scaled Emitter Dimensions . . . . . . . . . . . . . . 13

2 Estimated Performance Profile of Colloid Thruster . . . . . . . . . 37 


\section{LIST OF FIGURES}

Figure

Page

1 The Basic Electrospray Configuration . . . . . . . . . . . . . 4

2 The Three Emitter Types . . . . . . . . . . . . . 6

3 Cone-jet Formation of Glycerol . . . . . . . . . . . . . . . 8

4 Time-Averaged Images of Electrospray Emission Increasing in Voltage From $1.2 \mathrm{kV}$ to $1.7 \mathrm{kV}$ in 50V Increments From Left to Right . 9

5 Thruster Schematic . . . . . . . . . . . . . 15

$6 \quad$ Emitter Plate Schematic . . . . . . . . . . . . . . 18

$7 \quad$ Grid Schematic . . . . . . . . . . . . . . . . . 19

$8 \quad$ Module B Assembly . . . . . . . . . . . . . . 20

$9 \quad$ Assembled Thruster f. . . . . . . . . . . . . . . . . 21

10 The Student Vacuum Chambers . . . . . . . . . . . . . . . 24

11 Chamber Schematic ................. 26

12 Electrical Schematic . . . . . . . . . . . . . . . 27

13 Test Assembly . . . . . . . . . . . . . . . . . . . . . . . . . . . 28

14 Collection Plate . . . . . . . . . . . . . . . . 34

15 Cone-jet Formation on an Emitter . . . . . . . . . . . . . . 35

16 Pulsating Emission Mode . . . . . . . . . . . . 35

17 Failure Mode ... . . . . . . . . . . . . 36

18 Theoretical Thrust Compared to Estimated Actual Thrust . . . . . 38 
Chapter 1

\section{INTRODUCTION}

As CubeSats and other small satellite platforms have increased in popularity and demand, there has been a push for the development of propulsion systems to maneuver and maintain orbital position of these satellites. Electrospray technology can be implemented to create thrusters with fine precision and designed to fit the small form factors required to operate on these platforms. Electrospray thrusters operate by accelerating charged particles from an electrified liquid through an electrostatic field [1]. There are three emitter types to choose from: internal capillary, externally wetted, and bulk porous. Each emitter type comes with their advantages and disadvantages. There are also many different types of propellant to choose from. These design choices dictate the efficiency and performance of the thruster.

While research into this technology has been in existence for decades, there is an increasing pedagogical need to have engineering students become familiar with advanced electric propulsion systems as it is the University's duty to keep their curriculum as up to date as possible. This thesis will discuss the foundational principles behind the electrospray operations. Likewise, the design and implementation of an electrospray thruster to be used as a laboratory activity for post-secondary engineering students will be discussed. As such, the design for the thruster needs to be geared towards being an educational tool rather than a thruster optimized for maximum performance. 


\subsection{History and Application}

Investigation into electrospray technology for aerospace applications began in the 1960s. The primary goal was to discover an alternative to ion thrusters [1]. Preliminary analysis showed that the higher molecular mass of the propellant expelled by an electrospray thruster would give it a higher thrust density than ion thrusters and thus producing higher thrust performance [1]-[4]. While the theory was successfully implemented, a new set of problems was introduced. These thrusters required anywhere from 10 to $100 \mathrm{kV}$ in order to achieve successful electrospray operation. This led to thermal mitigation problems when integrating the thruster into satellite systems [1], [2]. Such thermal issues and power requirements caused these thrusters to be viewed as more effort than they were worth, and, consequently, the development of electrospray for aerospace purposes were put on hold [1], [2], [5], [6].

With the rise of small satellites and advancements in technology, electrospray thrusters have seen a resurgence of interest. The increased prevalence of small satellites has created a demand for low-thrust, high-precision thrusters [1], [2], [4], [7]. Additionally, biological scientists have continued research into electrospray technology as a means for extraction of charged biological macro-molecules for detailed mass spectroscopy [1], [5]. These advancements have brought the voltage requirements down to 1 to 5 $\mathrm{kV}$, which is more manageable [1]-[4], [8], [9].

Currently, the only flight model of a colloid thruster is on board the LISA Pathfinder mission [8]. The Laser Interferometer Space Antenna, or LISA, mission aims to measure gravitational waves using three spacecraft that relay laser beams back and forth between each other. The LISA Pathfinder mission tests key technologies such as electrospray thrusters. In order to accurately measure the gravitational waves, there needs to be near zero disturbances or noise on board these spacecraft [8], which is 
any disturbance on the spacecraft generated by any mechanical or electrical systems while it is operating. To accomplish position maintenance and attitude stability, NASA JPL and Busek have collaborated on the development of a flight qualified electrospray thruster for the mission. The demonstration of the thruster on board the LISA Pathfinder proves the success of the thruster operation as well as the points of failure. This data is now being used to develop the next iteration of electrospray thrusters to be used in flight [8], [10], [11].

\subsection{Electrospray Theory}

The formal definition of an electrospray thruster is a type of thruster that accelerates charged particles produced from electrified liquid surfaces through an electrostatic field [1]. To create this electrostatic field, a strong electric potential is applied between an extractor grid and the end of an emitter tip containing a liquid propellant. A Taylor cone forms, concentrating the electric field strength near the cone tip, and, at a sufficient electric field strength, charged droplets will be extracted from the tip of the cone [2], [5], [12]. The charged droplets are accelerated by the electrostatic field. This process is illustrated in Figure 1. In some cases, there is also an accelerator grid after the extractor grid, which is also shown in Figure 1. An accelerator grid is not needed to generate thrust as their purpose is to focus the beam and add more voltage to increase the thrust produced [1], [6], [10]-[12].

As with other electric propulsion systems, the emitted propellant carries a charge and must be neutralized in order to prevent back flow and reattaching to the grid or any other part of the spacecraft. The most direct way to neutralize the flow is to integrate a neutralizer with the propulsion system [7], [9], [14]. Another way of neutralizing the flow is to have multiple emitters that alternate polarity of the emitted propellant. 


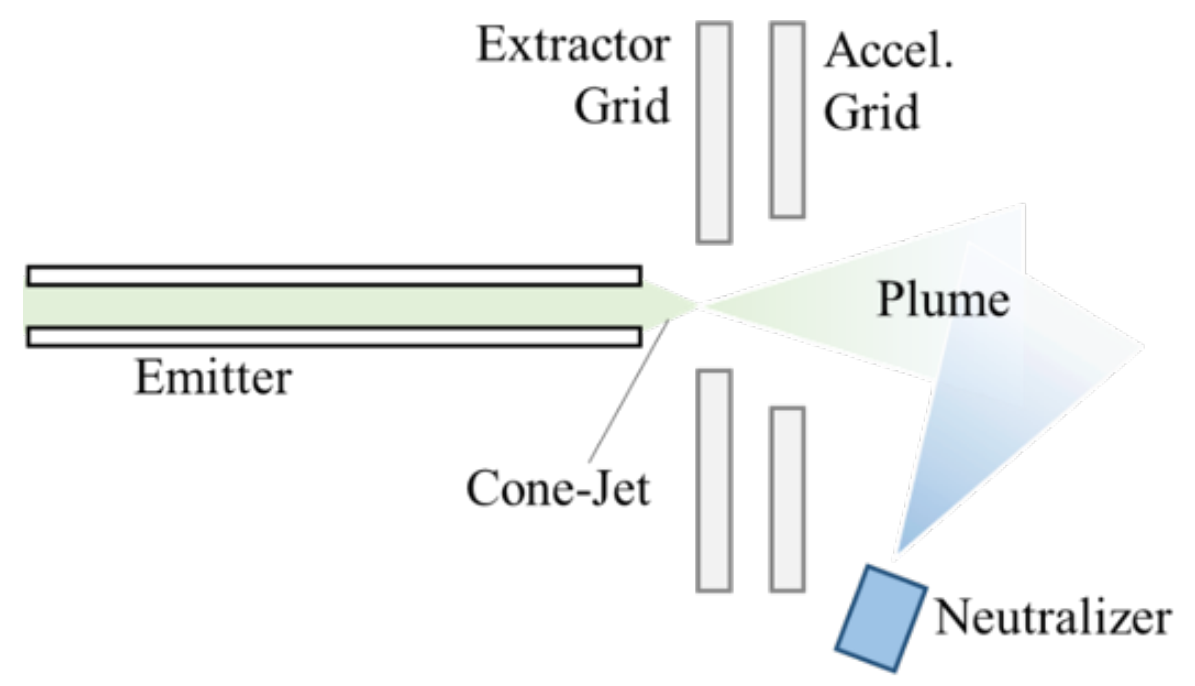

Figure 1: The Basic Electrospray Configuration [13] showing the movement of propellant through an emitter that gets extracted by an extractor grid. The extraction occurs by applying an electric potential between the emitter and extractor. The accelerator grid that follows is not necessary, but it helps focus the beam and increases the thrust produced by increasing the electric potential. The neutralizer at the end neutralizes the flow to help detach the flow from the thruster.

This way, the flow becomes quasi-neutral without the aid of a neutralizer. In order to achieve negatively biased droplets, the propellant must be doped with certain salts $[5]$.

An electrospray thruster's thrust $(\mathrm{T})$ and specific impulse $\left(\mathrm{I}_{\mathrm{sp}}\right)$ performance calculations are given by Equation 1.1 and Equation 1.2, respectively, [7]-[9]:

$$
\begin{gathered}
T=\dot{m} v_{\mathrm{e}}=\dot{m} g I_{\mathrm{sp}} \\
I_{\mathrm{sp}}=\frac{1}{g} \sqrt{2 \frac{q}{m} V_{\mathrm{a}}}
\end{gathered}
$$

where $v_{\mathrm{e}}$ is the propellant exit velocity, $\dot{m}$ is the mass flow rate of the propellant, $g$ is the acceleration due to gravity on Earth, $\frac{q}{m}$ is the charge per mass of the propellant, and $V_{\mathrm{a}}$ is the voltage applied to the extractor-emitter system and the accelerator grid 
if there is one.

The propellant can be extracted as charged droplets or as ions. Ions have a better charge per mass ratio because ions have a charge that is a scalar multiple of the elementary charge and a mass that is of the atom itself. This gives the system a high $\mathrm{I}_{\mathrm{sp}}$. However, since $T$ is dependent on $\dot{m}$, and ions have a very low mass compared to droplets, the ion emission mode produces less thrust than the droplet mode. Conversely, droplets will give lower specific impulses but higher thrusts because the droplets hold the same charge but have a mass much greater than an ion [2], [4], [6]. The decision for which mode to operate in is dependent as two which configuration is more optimal for mission success.

From this, three main subgroups emerge: colloid thruster, field emission electric propulsion (FEEP), and ionic liquid ion source (ILIS). Colloid thrusters accelerate charged droplets and, under special circumstances, ions and use solvents such as doped glycerol and formamide as propellant. A doped propellant means that another substance, usually a salt, has been dissolved in the propellant to increase the electrical conductivity of the propellant to lower the voltage needed to induce emission. This is advantageous by lowering the voltage required, which lowers the minimum thrust produced for satellites that need fine thrust control. Propellants that allow for a lower minimum voltage have higher $\frac{q}{m}$ values, thus maintaining a high $\mathrm{I}_{\mathrm{sp}}$ of the system. FEEP utilizes liquid metals, usually cesium or indium, to produce and accelerate positively charged metallic ions. ILIS uses room-temperature molten salts to produce and accelerate ion salt beams, which are mixtures of ions and droplets [1]. 


\subsection{Emitters}

The emitter design dictates the movement of the propellant from the reservoir to the cone-jet formation. There are three main emitter types: internal capillary, externally wetted, and bulk porous, as shown in Figure 2 .

An internal capillary emitter draws propellant from a reservoir and passively moves up the emitter due to capillary forces. In order to aid the formation of a Taylor Cone, the tips of these emitters are chamfered at an angle that follows the contour of the Taylor cone that is expected to form. An externally wetted emitter draws the propellant over the emitter surface by capillary and surface tension forces and then expelled at the tip. A bulk porous is a blend between the other two emitters. The porous materials used to make these emitters have small internal capillaries that allow the propellant to be drawn up through capillary forced. These pores exit to the surface so that the propellant is then secreted to cover the emitter, and then expelled at the tip [15].

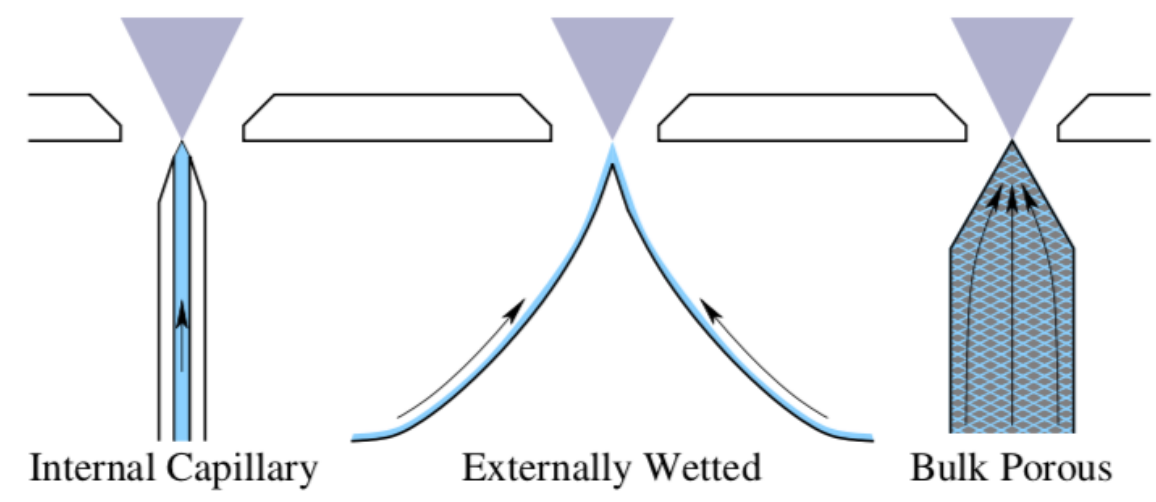

Figure 2: The Three Emitter Types [15] are internal capillary, externally wetted, and bulk porous. All of these utilize capillary action and surface tension forces to bring the propellant to the tip in order to be expelled.

With the development of Micro-Electro-Mechanical Systems, or MEMS, externally wetted is becoming the most preferred method of emitting the propellant [15]. The 
main process of manufacturing the emitters is through chemical etching. Chemical etching allows for a controlled and uniform method of carving out microscopic entities of the emitter. The manufacturing process of externally wetted emitters also allows different geometries to be designed to help maximize performance. These entities maximize performance by helping the movement of propellant as they allow optimized ridges for the propellant to travel up to the emitter tip [5], [6], [15]. Along with these benefits, MEMS can optimally integrate the extractor grid and the emitter array [6], $[15]$.

The propellant can either be actively fed or passively fed to the emitters. In a passively fed system, the mass flow rate of the propellant is dictated by the inherent movement of the propellant through the emitter [3]. This is advantageous as there is reduced complexity in the system. However, the mass flow rate is constrained by how much propellant is being emitted, and it can be difficult to achieve the desired propellant mass flow rate [3]. An actively fed system is where the propellant is fed into the emitters byway of a mechanical pump or by being pressurized [8]. This system is then able to control the mass flow rate of the system, but this adds complexity as additional pipes, valves, and controllers need to be implemented [8].

\subsection{Taylor Cone}

The fundamental aspect of the extraction of the droplets comes from the Taylor Cone structure of an electrified liquid. A Taylor Cone is where the liquid is extended beyond the tip of the emitter and forms a cone-like shape. The Taylor Cone is an idealized occurrence when the electric field becomes infinitely large at the tip while becoming infinitesimally small in physical size [12]. One of the assumptions for this situation is that there is no flow through the cone. This assumption falls apart considering that 
flow is required in order to produce thrust. This extraction formation is now referred to as the "cone-jet" mode [12], which is depicted in Figure 3. Nonetheless, the terms "Taylor Cone" and "cone-jet" are often used interchangeably.

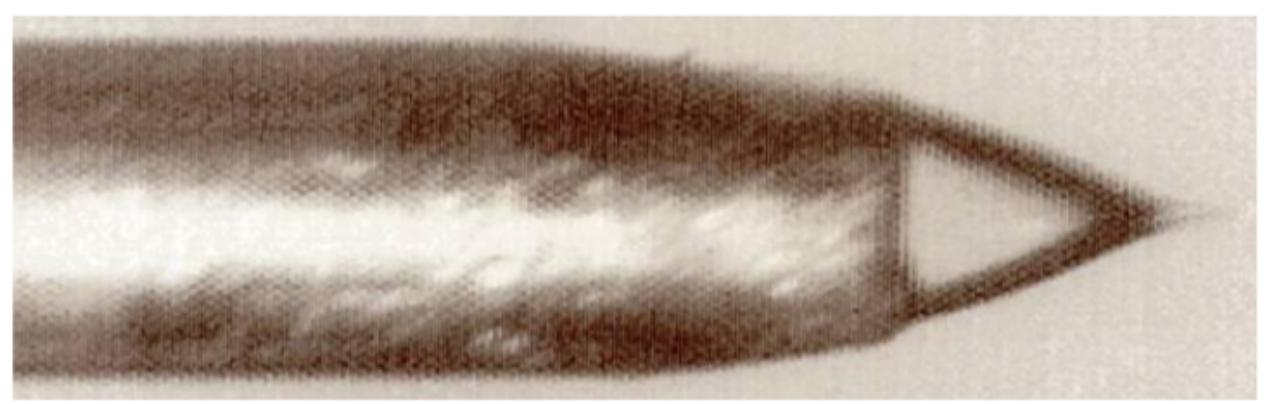

Figure 3: Cone-jet Formation of Glycerol [14] is created with a chamfered internal capillary emitter. The chamfered edge aid the formation of the cone-jet as it follows the contour of the cone. The propellant then gets pulled off of the cone into a well formed jet, as shown to the right of the figure.

While the cone-jet operation is what is desired, there are other transient modes that occur as well when the electric field in the emitter-extractor system is either too strong or too weak. The different modes in order of ascending voltage are pulsating emission mode, transition pulsating emission mode, steady cone-jet emission mode, whipping, whipping and multi-jet emission mode, and multi-jet emission mode. The effects on the emission beam are shown in Figure 4.

As seen in Figure 4, the images on the left have a large, bulbous cone-jet formation. These images depict an electric field that is too weak to create a steady cone-jet formation, which creates pulsating and transition pulsating emission modes [11]. Conversely, the images on the right have a small, sharp cone-jet formation. These images depict the whipping, whipping and multi-jet, and multi-jet emission modes that occur when the electric field is too strong. When this occurs, multiple emissions sites are formed from the cone-jet. Notice how the emitted propellant creates a V-shaped spray [11]. This shape causes the propellant to loose efficiency as the thrust is not 


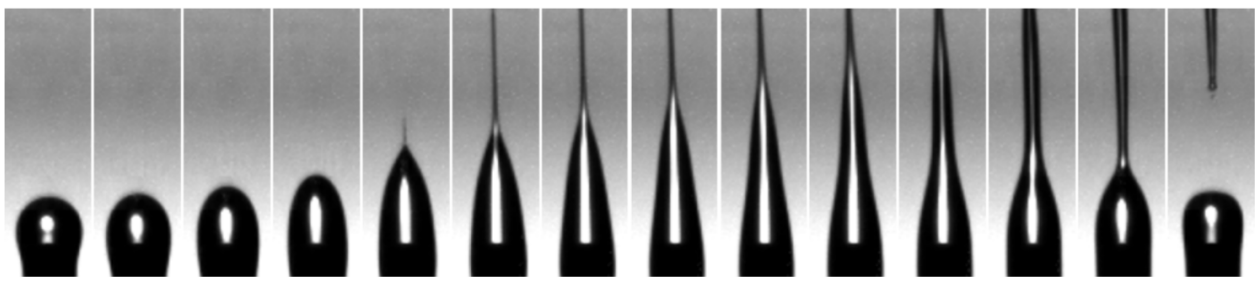

Figure 4: Time-Averaged Images of Electrospray Emission Increasing in Voltage From $1.2 \mathrm{kV}$ to $1.7 \mathrm{kV}$ in $50 \mathrm{~V}$ Increments From Left to Right: [10] This experimentation uses ethanol in atmosphere using an internally capillary emitter with a volumetric flow rate of $0.75 \mathrm{~nL} / \mathrm{s}$. These images show how the emission form is affected when the electric field is too weak (left), ideal (middle), and too strong (right). The emission modes, from left to right, are pulsating, transition pulsating, steady cone-jet, whipping, whipping and multi-jet, and multi-jet emission modes. Any emission mode that is not a steady cone-jet formation will cause lifetime issues of the thruster.

concentrated in one direction, and, depending on the width, the spray could hit the extractor or accelerator grids [10]. The middle-left to middle images demonstrate the proper steady cone-jet formation, which is the emission mode that is to be desired [10], [11]. Any emission mode that is not steady cone-jet emission causes the lifetime of the thruster to shorten as it increases the risk of grid impingement, which is when propellant bridges the circuit between the extractor and emitter or the extractor and accelerator grids causing the emitter-extractor system to short and make further propellant emission impossible [10], [11].

\subsection{Thesis Scope}

As the theory of electrospray operations continue to be researched, new technology is created and, thus, meeting the demands for small satellite propulsion. Emerging technology creates the need for curriculum to be updated. The objective of this thesis is to design, construct, and implement a laboratory activity for the Spacecraft Propulsion curriculum at California Polytechnic State University, San Luis Obispo (Cal Poly), 
that teaches electrospray theory in accordance with the university's "Learn by Doing" pedagogical philosophy. The design for the thruster will be driven by creating opportunities for visual and tactile interactions for the post-secondary students with the system as well as provide the means for meaningful data collection and data analysis. The students are expected to measure the mass of propellant emitted. From this, the students are then expected calculate the thrust and specific impulse of the thruster. After having operated the thruster, the students are then expected to evaluate the performance of the thruster and recommend design changes to improve the thruster. Additionally, the laboratory activity needs to be completed within two hours and fifty minutes per the allotted time for laboratory activities at Cal Poly. Success for the system will be the development of a thruster that uses electrospray function to produce repeatable data. This will be proven by the generation of a thrust profile and various performance parameters of the thruster, which can be the basis of expected results from the students. This thruster is not designed to maximize the thrust produced or efficiency. Additionally, this thruster will not go on to be flight qualified or be used to investigate new techniques or phenomenon. 
Chapter 2

\section{THRUSTER DESIGN}

The main objective is to design and construct an apparatus that can serve to demonstrate the electrospray theory and can be operated by undergraduate students with the current equipment that exists in the space environments laboratory designated for undergraduate use. As it is an educational tool, the design constraints are to keep manufacturing, operating, and maintaining the thruster costs to a minimum. The importance of the design also lies in embodying the "Learn by Doing" pedagogical philosophy rather than maximizing thrust or $\mathrm{I}_{\mathrm{sp}}$. This means that the physical nature of the thruster needs to be large enough to differentiate the components of the thruster by the naked eye in order to demonstrate how each aspect contributes to the whole of the thruster operation. While maximizing performance is not important, the thruster still needs to be able to produce meaningful and repeatable data in order for the students to be able to learn how to experimentally analyze an electrospray thruster.

Emphasizing the "Learn by Doing" philosophy is important because experiential learning is the most powerful form of learning as it is the natural way of learning [16]. This means that any knowledge gained through interactive exercises make a deep and lasting impression. As such, Cal Poly is committed to improve student experience by including learning experiences that align with industry and current technology. 


\subsection{Design Decisions}

The key drivers in the design of this thruster are high visual and tactile interactions with the system to create an experiential learning platform [16] as well as keeping a low cost to produce and maintain the thruster. This drives the decision to use the colloid thruster definition of electrospray thrusters. The thruster is also equipped with a planar array of internal capillary emitters and uses glycerol as the propellant in the droplet mode.

Glycerol is a widely available and inexpensive liquid to obtain. It has a wide range of commercial uses including soaps, lotions, and as a low-fat sugar substitute in food and beverages [17]. The non-toxic nature of glycerol is beneficial as it will not pose a threat to the health and safety of the students and instructors that will be operating this lab. Additionally, by not doping the glycerol, there are no added expenses in purchasing the salts, no additional time being spent in doping the glycerol, and no additional health risks to students in handling the salts. This in turn helps the activity operate in the time constraints of the lab and reduce preparation time for the instructors. While doping the propellant is not being implemented in the lab activity, it would still be important to discuss the theory and practice of propellant doping.

Due to the micro-sizing of colloid thrusters in order to be used in small satellites, microscopes are sometimes needed to observe individual components of the thruster. The colloid thruster for the laboratory activity is scaled up in size in order to have a visual impact on the students without the use of a microscope. This need leads to the decision of using internal capillary emitters as they are larger than externally wetted emitters and are less expensive than bulk porous emitters. Additionally, internally wetted emitters can maintain performance better when scaled up compared 
to externally wetted or bulk porous, which perform better when scaled down. The emitter array design of this thruster is a scaled array design from Dr. VelazquezGarcia's planar array design for his $\mathrm{PhD}$ dissertation [5]. The decision to base the array design on a research thruster rather than a commercially produced thruster is driven by the fact that electrospray schematics for private companies are not widely published due to proprietary information protection. Additionally, MIT is the leading research institute into electrospray thrusters. As such, the designs that come from MIT research have decades of design heritage behind them. The scaling scheme is demonstrated in Table 1.

Table 1: Scaled Emitter Dimensions: This table gives the numerical values of the original emitter array design by Dr. Velazquez-Garcia, and how these values are then scaled to create the array design for this thruster.

\begin{tabular}{|l|l|l|l|}
\hline Entity & PhD Design [5] & $\begin{array}{l}\text { Non-Dimensional } \\
\text { Value }\end{array}$ & Scaled Dimension \\
\hline Emitter Diameter & $154 \mu \mathrm{m}$ & 1 & $1.37 \mathrm{~mm}$ \\
\hline Emitter Spacing & $500 \mu \mathrm{m}$ & 3.25 & $4.45 \mathrm{~mm}$ \\
\hline Row Spacing & $750 \mu \mathrm{m}$ & 4.87 & $6.67 \mathrm{~mm}$ \\
\hline
\end{tabular}

The standard by which the entities are scaled is the emitter diameter because the size of the emitter for this thruster is known to be an eighteen gauge needle. As such, all the entities in the research thruster are divided by its emitter diameter of $154 \mu \mathrm{m}$. This results in the non-dimensional values. Then, the non-dimensional values are multiplied by the lab activity's thruster emitter diameter of $1.37 \mathrm{~mm}$. This method ensures that all sizing and spacing is properly scaled in proportion to how much the emitters are scaled.

By using a planar array modeled after an existing configuration, the students are able to interact with a system similar to what they would see in technological research and development rather than just an arbitrarily arranged configuration. A planar array 
allows for more emitters to be implemented, which results in more emission and, in turn, a higher thrust. A higher thrust increases the propellant emitted over a shorter amount of time. This helps keep the lab activity within the time constraint while also maximizing the amount of visible propellant emitted. This further contributes to the visual impact of the lab.

In keeping with the desire to create a system similar to that which would be encountered within industry, a neutralizer and acceleration grid are added. The neutralizer is a simple tungsten-filament neutralizer with its own power supply. Given that optimal neutralization isn't the focus for this demonstration, the voltage and current is set to where the neutralizer glows, which is an indicator that electrons are being emitted to neutralize the flow. The accelerator grid helps focus the jet as well as increases the thrust produced. This, too, adds to the visual impact for the students. The culmination of all these design choices resulted in the thruster design, as shown in Figure 5.

An important design concern is how to maintain equal distribution of propellant to all of the emitters. The decision was made to place the emitters in a horizontal orientation as to eliminate the force of gravity from affecting the thruster's propellant emission. By having a horizontal orientation, gravity still poses an issue in that as the propellant is consumed, gravity pulls the propellant down rendering the top emitters unable to draw propellant from the propellant cavity. To mitigate this issue, a twomodule design is implemented, as labeled in Figure 5. The bottom module, Module $\mathrm{B}$, houses the emitter planar array, a propellant cavity, and both the extractor and accelerator grids. The top module, Module A, serves as the main propellant reservoir. The modules are connected by a pipe. With this design, the main propellant reservoir will deplete in order to keep the propellant cavity full, thus ensuring that there is equal distribution of the propellant across all emitters. By utilizing gravity to help keep the 


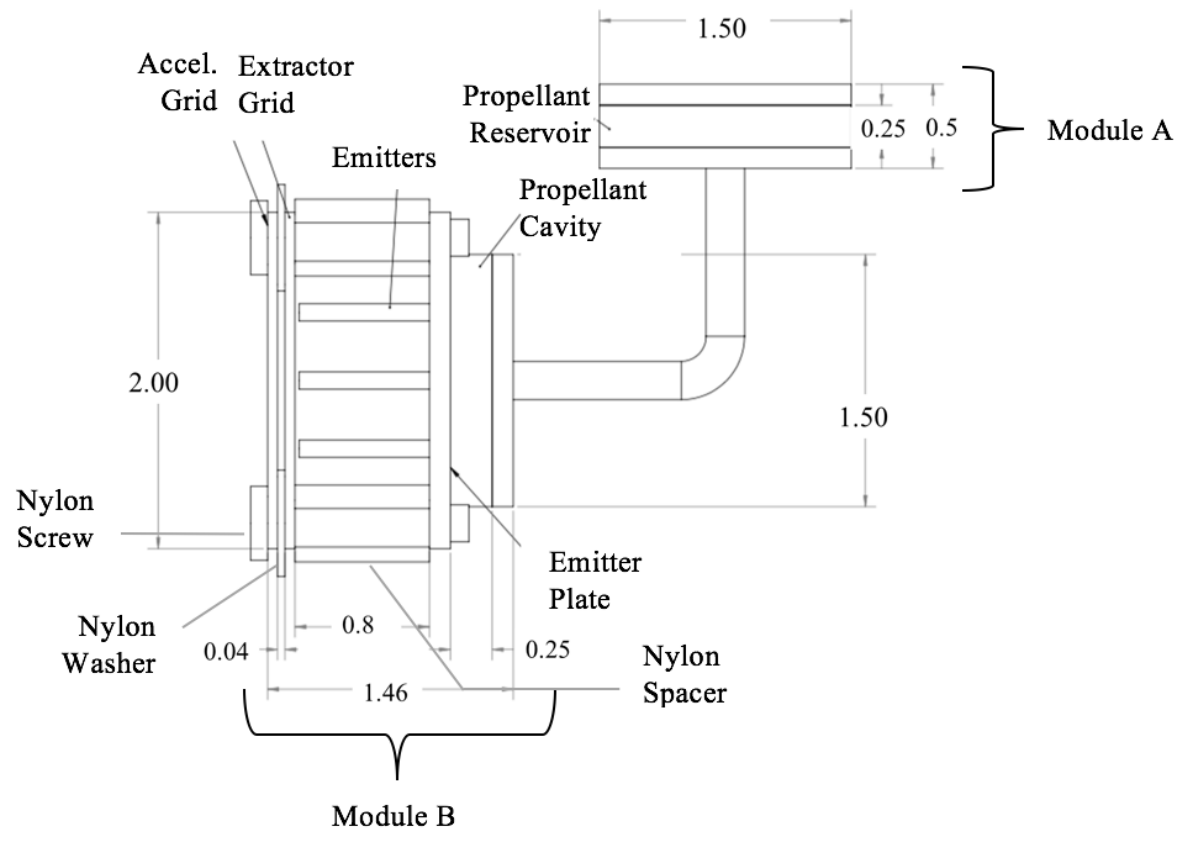

Figure 5: Thruster Schematic: The thruster that has been designed with dimensions and labels. Additional part schematics are available in Appendix A.

propellant, this isn't a strictly passive propellant feed. Rather, it is a gravity feed. A gravity independent feed is not necessary to develop for this thruster as it will not be flight qualified. However, it should be discussed with the students, and future work can be done to develop a true passive feed that is gravity independent. Additional schematics for the parts and assembly are available in Appendix A.

\subsection{Analysis}

The ideal thrust $(\mathrm{T})$ produced by electrospray thrusters as a function of the electric field is shown in Equation 2.1 [1]:

$$
T=A \frac{\epsilon_{0}}{2}\left(\frac{4}{3} \frac{V_{\mathrm{a}}}{d}\right)^{2}
$$


where $A$ is the cross-sectional area of the emitter tip, $\epsilon_{0}$ is the permittivity of free space, and $d$ is the distance from the emitter tips to the grid. The voltage applied $\left(V_{\mathrm{a}}\right)$ is constrained by the power supplies designated for this activity. The emitter area is known, as well. This leaves the distance remaining in the design space. In order to increase the likelihood of successful operation at low voltages, the distance between the grids and emitters needs to decrease.

The power requirement needs to be taken into consideration as well since power supplies have power consumption limits. Power follows Ohm's law, power equals current times voltage, where the voltage is the applied voltage, $V_{\mathrm{a}}$, and the current is the current carried by the cone-jet, $I_{\mathrm{b}}$, which can be found by using Equation 2.2 [2], [5], [12]:

$$
I_{\mathrm{b}}=f(\epsilon) \sqrt{\frac{\gamma K Q}{\epsilon}}
$$

where $f(\epsilon)$ is an empirical constant, $\gamma$ is the surface tension of the propellant, $K$ is the conductivity of the propellant, $Q$ is the volumetric flow rate of the propellant, and $\epsilon$ is the relative permittivity of the propellant. The empirical constant, $f(\epsilon)$ was developed by Fernandez de la Mora to accurately represent different dependencies on conduction contributions and the effects of internal fields. The quantity $f(\epsilon)$ has a value of 18 for $\epsilon>40$ [2], [5], [12]. By knowing that the propellant is glycerol, all of these parameters, except for Q, can be found in literature [17], such that $\epsilon$ is $42.8, \gamma$ is $0.0625 \mathrm{~N} / \mathrm{m}$, and $K$ is $100,000 \mathrm{Si} / \mathrm{m}$. The volumetric flow rate, Q, can be found using Equation 2.3 [2], [5], [12]:

$$
Q=\frac{\gamma K}{\epsilon\left(\frac{q}{m} \frac{\rho}{f(\epsilon)}\right)^{2}}
$$


where $\frac{q}{m}$ is the charge per mass ratio. The maximum $\frac{q}{m}$ for the propellant can be found in Equation 2.4 [12]:

$$
\left(\frac{q}{m}\right)_{\max }=\rho F \times 1000 c_{\mathrm{d}}
$$

where $F$ is Faraday's constant of $96500 \mathrm{C} / \mathrm{mol}$ and $c_{\mathrm{d}}$ is the dissociated part of the solution's equivalent normality. This can be found by dividing $K$ by the mobility parameter, which is $15(\mathrm{Si} / \mathrm{m}) /(\mathrm{mol} / \mathrm{L})$ [1]. Both Equation 2.3 and Equation 2.4 are theoretical calculations based on the propellant properties, which are the maximum $Q$ and $\frac{q}{m}$ that can be produced by the propellant. When calculating $Q$ and $\frac{q}{m}$ from experimental data using Equation 2.5 and Equation 2.6 [1]:

$$
\begin{gathered}
Q=\frac{\dot{m}}{\rho} \\
\frac{q}{m}=\frac{I_{\mathrm{b}}}{\dot{m}}
\end{gathered}
$$

where $\dot{m}$ is the mass flow rate of the propellant and $\rho$ is the density of the propellant.

Beam currents are typically on the order of $\mu \mathrm{A}$ and are directly proportional to the number of emitters that are installed. For the 10-emitter design, the power requirement is $2.4 \mathrm{~W}$, which comfortably less than the power limits of the designated power supplies of $10 \mathrm{~W}$. 


\subsection{Manufacturing}

Maintaining uniformity is key for successful operation of the thruster. This drives the decision to use prefabricated nylon spacers, washers, screws, and stainless steel blunt-end medical needles that can be modified to be internal capillary emitters, as shown in Figure 5. All of these materials are inexpensive to purchase. The nylon spacers help keep the extractor and accelerator grids at a uniform distance from the emitters. The nylon washers keep the accelerator grid uniformly spaced from the extractor grid while keeping it electrically isolated and minimizing the distance, $d$, from the emitters. The nylon screws keep the accelerator grid, extractor grid, and nylon spacers assembled while maintaining electric isolation.

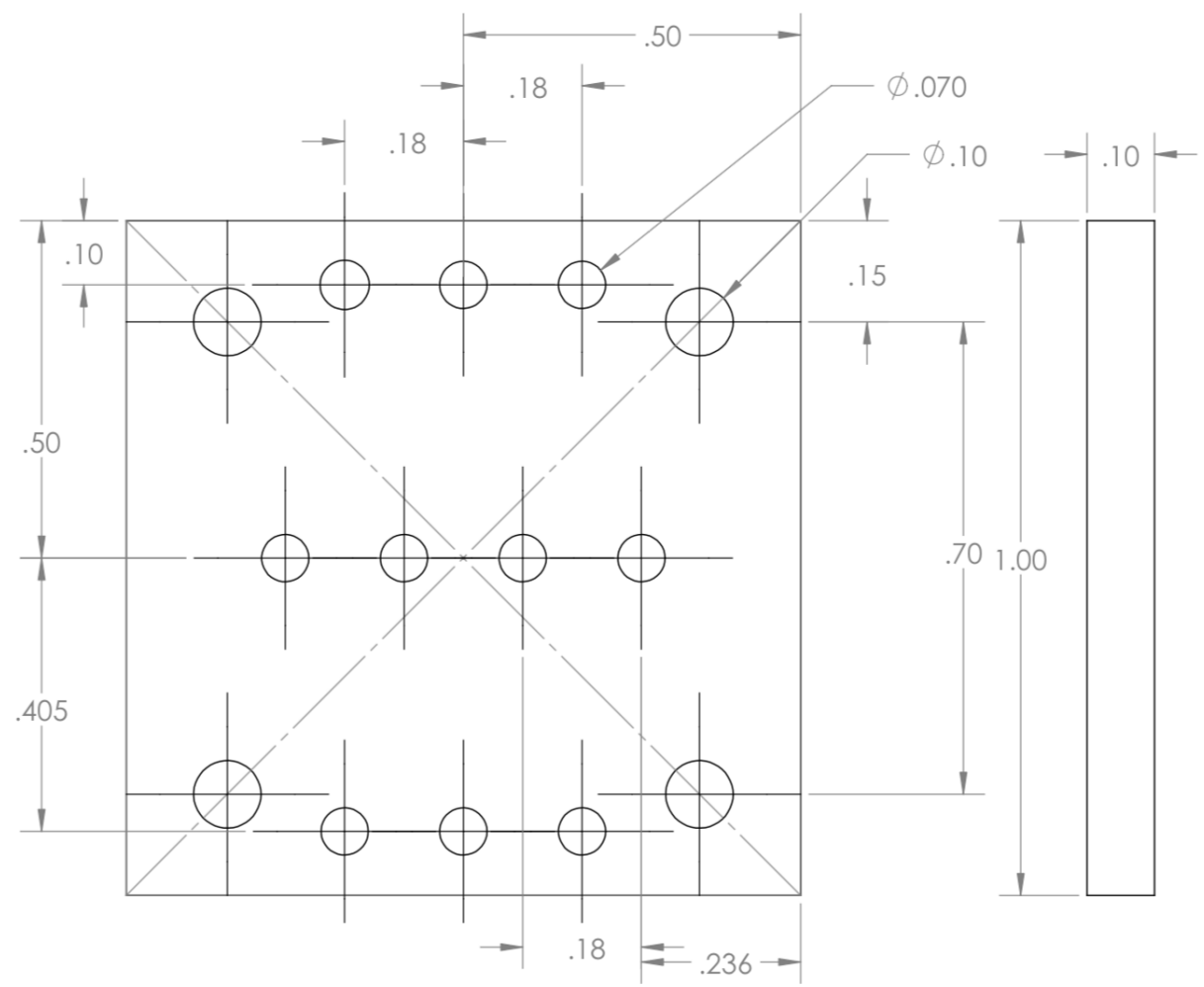

Figure 6: Emitter Plate Schematic: The emitter plate is designed to maintain a precise emitter array pattern. Blunt-end surgical needles are cut to length and soldered into these holes. The four holes in each corner are for screws to hold the emitter plate in place. 
A small plate is designed and CNC-machined at Cal Poly out of stainless steel with holes for the emitters to be soldered into in order to keep the emitters properly spaced with a uniform voltage applied across all 10 emitters. Stainless steel is chosen as it is in abundance and inexpensive to obtain, which helped minimize cost. The medical needles are cut to a length of .755 in. such that the emitters are the length of the nylon spacers less the thickness of the nylon washers. This allows for the distance between the emitters and the extractor grid to be the same as the distance between the accelerator and extractor grids, which is $.045 \mathrm{in}$.

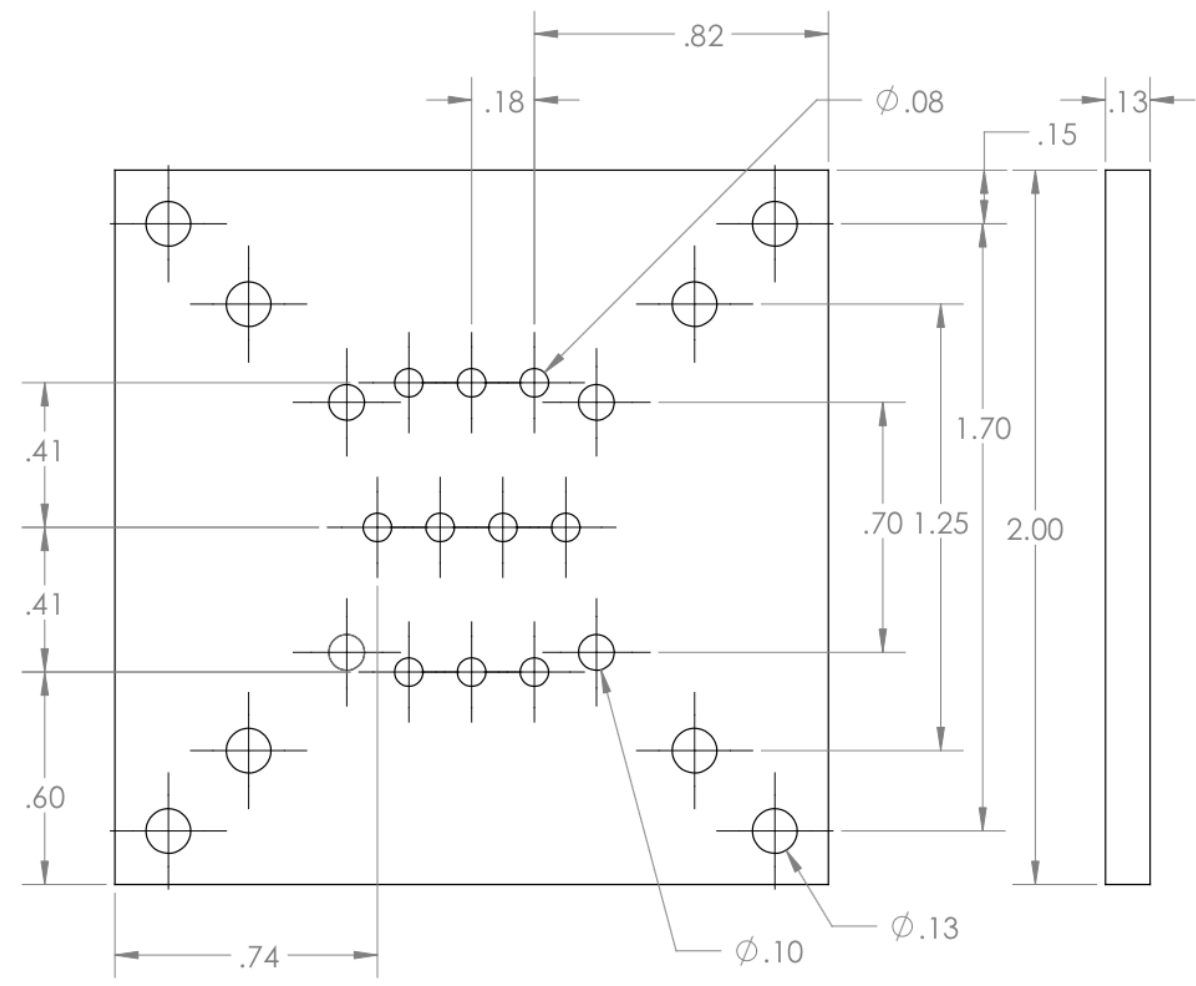

Figure 7: Grid Schematic: This schematic is the design for both the extractor and accelerator grids. The cluster of ten holes in the middle are where the the propellant is emitted through. The additional holes are for mounting and securing the grids to the thruster as well as mounting and securing the filament neutralizer to the accelerator grid.

The grids, as shown in Figure 7, are also CNC-machined at Cal Poly out of stainless steel. By having the emitters set by a CNC-machined plate, the holes for the grids 
are able to be aligned with the emitters with a tolerance of $\pm .005 \mathrm{in}$. Additional holes are made in the peripheral to allow for electrical lead attachments and neutralizer attachments as well as providing access for screw drivers to be used to tighten screws on the module.

The two modules are CNC-machined at Cal Poly out of high-density polyethylene, or HDPE. HDPE is an easy to manufacture plastic that is readily available and quite inexpensive. HDPE provides electrical isolation and minimizes the conductive surface area to reduce arcing. The modules follow a cap and body design that require screws to be held together, as shown in Figure 8 .

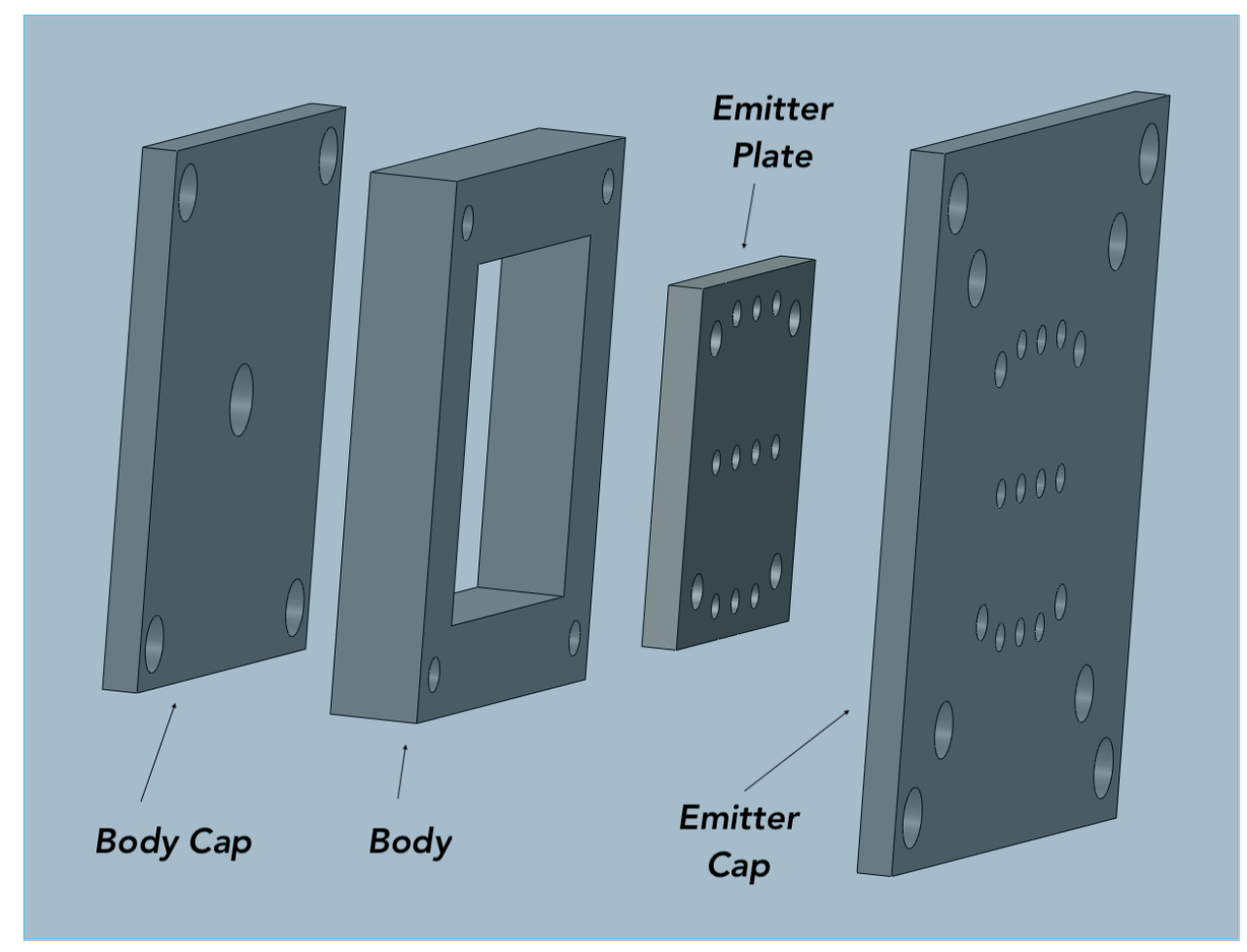

Figure 8: Module B Assembly: The exploded view shows how Module B comes together. The stainless steel emitter plate is placed inside of the HDPE casing to minimize the conductive surface area in order to reduce arcing.

The emitter plate is housed inside of the HDPE casing to limit the surface area of exposed metal to help prevent arcing, which is also demonstrated in Figure 8. To 
keep the propellant from leaking, silicone gaskets are cut to be placed at the assembly points, and a Swagelok pipe assembly is fitted to connect the two modules. This design creates easy disassembly to perform maintenance and a pedagogical opportunity to show the inner components of the thruster and how they work. Note that in Figure 8 the emitters are not placed into the emitter plate. The entire assembly of the thruster is shown in Figure 9.

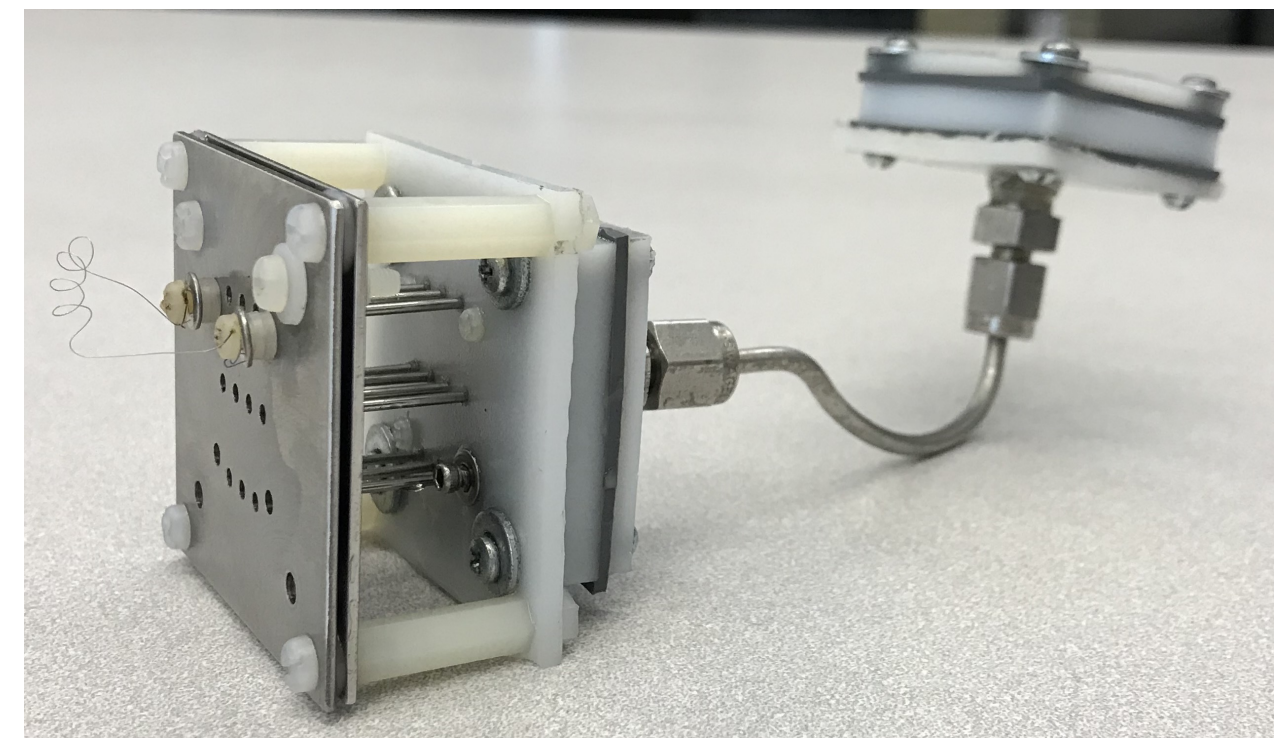

Figure 9: Assembled Thruster: The final product shown after all the manufacturing and assembly is completed.

\subsection{Limitations}

It is important to note that the emitters are soldered into the emitter plate by hand. This induces alignment errors as big as \pm 1 deg in each emitter to the system. These errors can reduce efficiency by as much as $50-60 \%$ as the cone-jet can send droplets onto the grids rather than through the grids, leaving behind a film of propellant on the extractor and accelerator grids. Additionally, this can cause an impingement on the grids, rendering the thruster inoperable. Grid impingement is likely to occur as the thruster gets used overtime. This can be fixed and prevented by cleaning the 
grids with isopropyl alcohol.

In addition to imperfections induced by hand soldering, stainless steel and soldering material do not naturally bond well with each other. An intermediary liquid, known as flux, needs to be used to help the two materials join together. While useful, flux is not always completely effective. This gives rise to having weak soldering joints in some of the emitters, which can bend or break leading to emitter misalignment, propellant leakage, and inability to carry the applied voltage. These joints should be checked and re-soldered if necessary to maintain performance.

While every effort is made to prevent propellant leakage, the propellant will still unintentionally leak to some degree from the emitters because of the low hydraulic resistance of the emitters. Unintentional leaking also occurs at the solder joints. Therefore, it is important to contain the leaking propellant in order to prevent it from contaminating the vacuum chamber and the vacuum pumps. The leaking propellant can also cause a grid impingement. Since the propellant is a conductive liquid, if the propellant ends up in a pool in between the grids, the circuit between the emitters, extractor grid, and accelerator grid can be bridged, thus rendering the thruster inoperable. 
Chapter 3

\section{METHODOLOGY}

One of the driving requirements is that this system can operate with the equipment available to the undergraduate students in the Space Environments and Testing Laboratory at Cal Poly. This restricts the equipment to the Student Vacuum Chambers, the two Glassman 1kV High Voltage power supplies, and a HP 20 V DC power supply. Furthermore, the design for the system should be kept simple to allow easy operation by undergraduate students and to promote the implementation of this system at other educational institutions. This section will describe the equipment, experimental set up, and the methodology to prove the system to be a functional and repeatable electrospray thruster.

\subsection{Laboratory Equipment}

The Space Environments and Testing Laboratory has two Student Vacuum Chambers (SVC), as depicted in Figure 10 available for undergraduate use. All of the experiments for this thruster will be completed in the second SVC, which is the one on the right in Figure 10. This is done in order to limit any unnecessary variables, such as chamber contamination and miscalibrated pressure gauges, induced by switching between the two chambers.

The SVC is a standard cylinder shape that is 2 feet tall and 1.5 feet in diameter. The chamber is connected to a Cacejen CRV 24 two-stage rotary vane vacuum pump and a Leybold 250iX turbo-pump. The rotary vane pump can reach a base pressure of 20-30 mTorr. This is sufficient enough, as the chamber only needs to reach 100 mTorr 


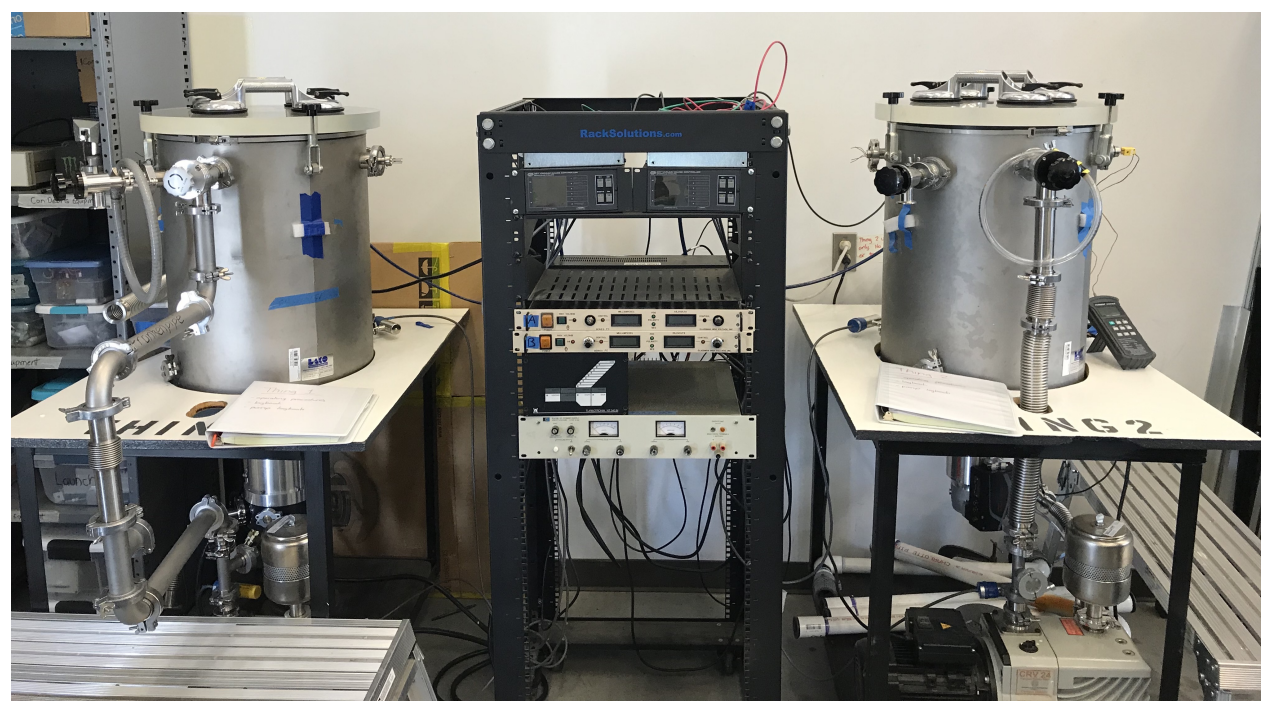

Figure 10: The Student Vacuum Chambers: These chambers are located in the Space Environments and Testing Laboratory that are intended for undergraduate learning and research. The chamber on the right is the one that is used for the testing of this thruster. The power rack in the middle contains the power supplies and the convectron gauge pressure monitors.

to prevent arcing with the smallest gap of .045 in, which is the distance between the extractor and accelerator grids of the thruster. [18]. With this, the turbo-pump will not be used. By not using the turbo-pump, the experimentation time is cut by an hour to an hour and a half, thus allowing for multiple tests to be completed with time to spare for trouble shooting in case of failure during the allotted two hours and fifty minutes. This too also saves on costs as a turbo pump does not need to be purchased if the vacuum chamber does not have one.

The chamber has three KF25 ports at the top of the cylinder and three KF16 ports at the bottom of the cylinder. Two of the KF25 ports contain high voltage electrical feed-throughs, which are insulated in order to prevent arcing in the feed-through. It is important that arcing in the feed-through does not occur because this would cause the applied voltage to not be delivered to the system.

The power rack between the two SVCs contains two convectron gauge pressure mon- 
itors, two Glassman High Voltage power supplies, and a 20V DC power supply respectively from the top of the rack to the bottom of the rack. The convectron gauge pressure monitors have digital readouts with dynamic precision readings. At base pressure, the monitors are precise to \pm 1 mTorr. The Glassman Series FC High Voltage power supplies each have a maximum voltage output of $1000 \mathrm{~V}$ and a maximum current output of $1 \mathrm{~A}$. The power supplies have digital readouts for both voltage and current, precise to $\pm 1 \mathrm{~V}$ and $\pm .1 \mathrm{~mA}$, respectively. The HP $6263 \mathrm{~B}$ DC power supply has a maximum voltage output of $20 \mathrm{~V}$ and a maximum current output of $10 \mathrm{~A}$. The power supply has analog readouts for both voltage and current precise to $\pm .5 \mathrm{~V}$ and \pm .5 A respectively. All of the power supplies have been properly grounded to the rack that is supporting them. The rack has a black insulating coating to ensure the power supplies do not accidentally shock the operator.

\subsection{Experimental Setup}

The chamber schematic, shown in Figure 11, shows how all the components outside of the chamber are integrated with all of the parts inside of the chamber. The two main considerations that require mitigation are arcing and propellant spray. As mentioned in Section 3.1, any arcing in the chamber introduces a short in the system, which renders the power supplies unable to deliver the power required to run the thruster. Additionally, propellant must be contained in order to not contaminate the chamber or damage the pumping system, which can become quite costly and affect lab operations.

To prevent arcing, the pump needs to be pumped below 100 mTorr, according to the stainless steel Paschen curve [18], given the thruster's smallest gap of .045 in., which is the gap between the extractor and accelerator grids. Additionally, the positive 


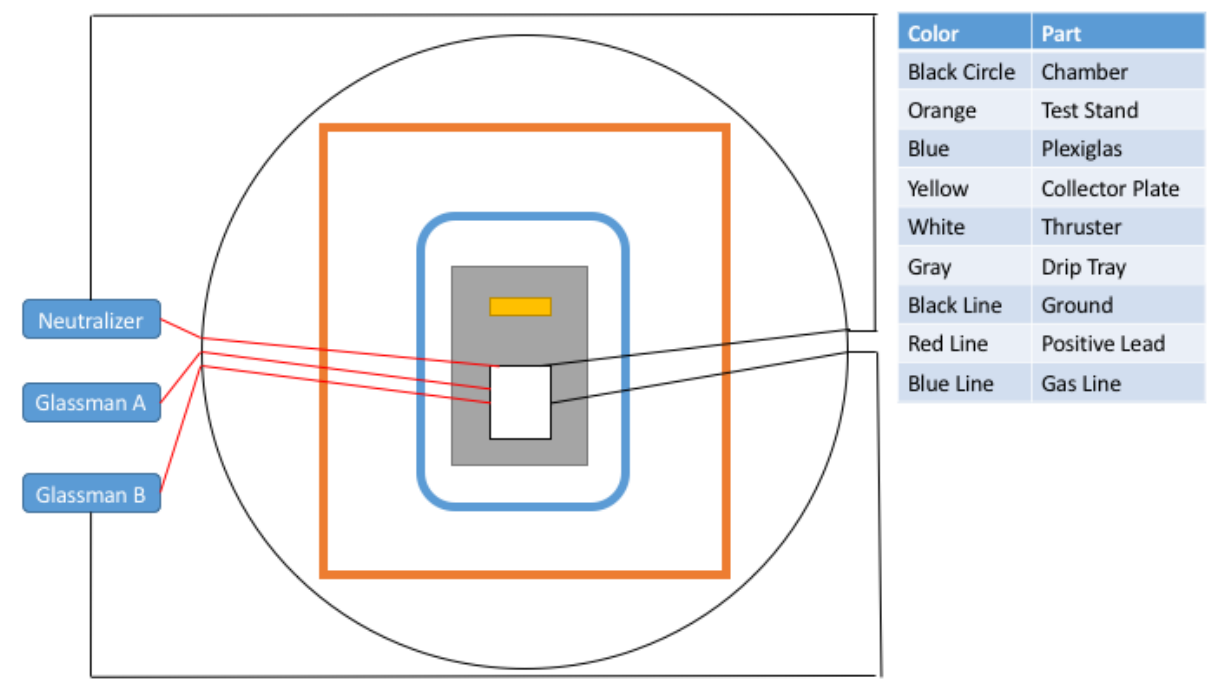

Figure 11: Chamber Schematic: This diagram demonstrates how all of the equipment outside of the chamber is integrated into the chamber. The legend to the right indicates how each part is represented in the diagram.

leads and ground leads of the Glassman High Voltage power supplies are fed into the chamber from opposite sides to prevent arcing in the electrical feed-through. One high voltage power supply is used to apply the potential across the emitter-extractor system, and the other high voltage power supply is used to apply the potential to the accelerator grid, which is illustrated in Figure 12. The low voltage power supply is set to $3 \mathrm{~V}$ and $1 \mathrm{~A}$ to activate the filament neutralizer. Special care must be taken in order to not apply too much current to the neutralizer or else it will break.

To prevent propellant from contaminating the chamber and vacuum pump, the thruster and the propellant collection plate are placed in a drip tray, which is also placed on a test stand. The full assembly is shown in Figure 13.

What is not pictured in Figure 13 is a vented Plexiglas box that gets placed over the whole system. This box helps protect the chamber in the event that any propellant that does not go towards the collection plate due to misalignment issues as discussed in Section 2.4. The box is clear in order to allow observations during the testing. 


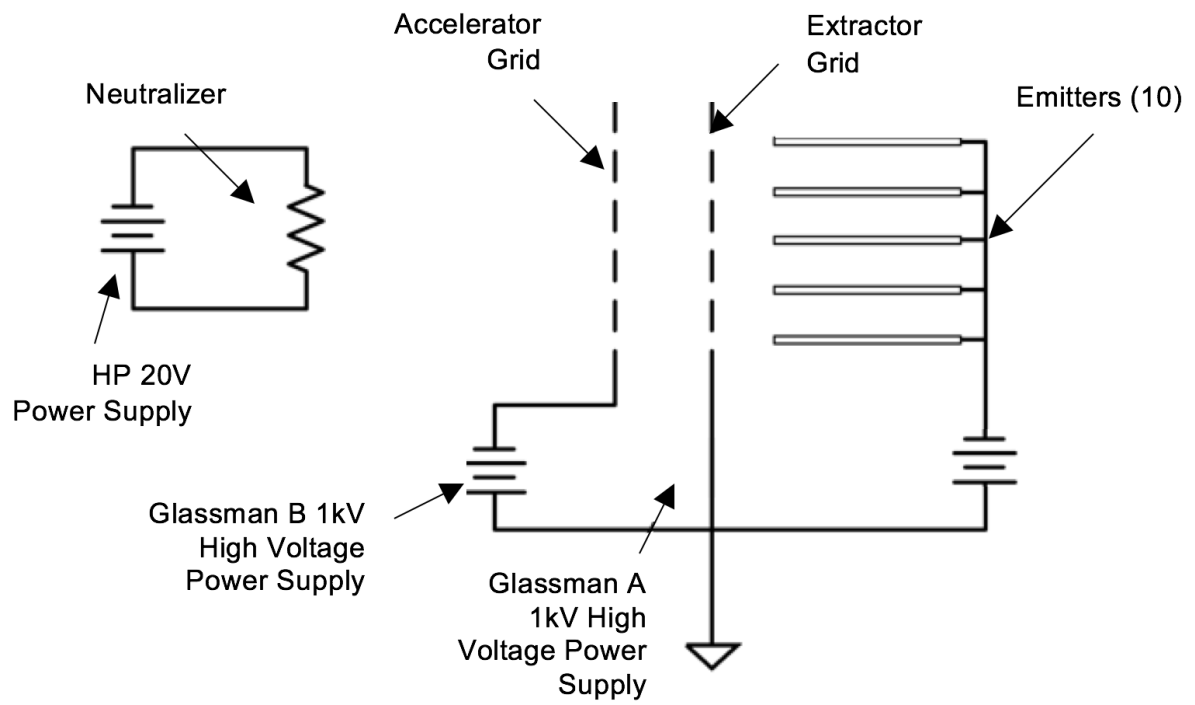

Figure 12: Electrical Schematic: This diagram illustrates how each power supply is connected to the thruster. One Glassman $1 \mathrm{kV}$ High Voltage is connected to the extractor and emitter system. The other Glassman $1 \mathrm{kV}$ High Voltage is connected to the accelerator grid. Both supplies are grounded to the rack. The HP $20 \mathrm{~V}$ DC power supply powers the fillament neutralizer.

Two items that are pictured in Figure 13 that have not been discussed are the tube in the top right of Figure 13 and the small acrylic block that is under the connecting pipe. The tube at the top may accommodate a propellant back pressure if an active propellant feed is desired. A propellant back pressure is not used in this experiment though because there is not a flow regulator available in the lab with the proper precision in order to keep the pressure from the nitrogen from being the dominant force that is moving the propellant. Acquiring this equipment and implementing it would add significant cost and complexity to the system. The small acrylic block is there to help the thruster sit more secure on the test stand and keep the thruster as level as possible.

Also shown in Figure 13, the two grids are cantilevering over the edge of the test stand. This is to help prevent any leaked glycerol from pooling in between the grids, causing any grid impingement. It is important to place the collector plate close enough to the 


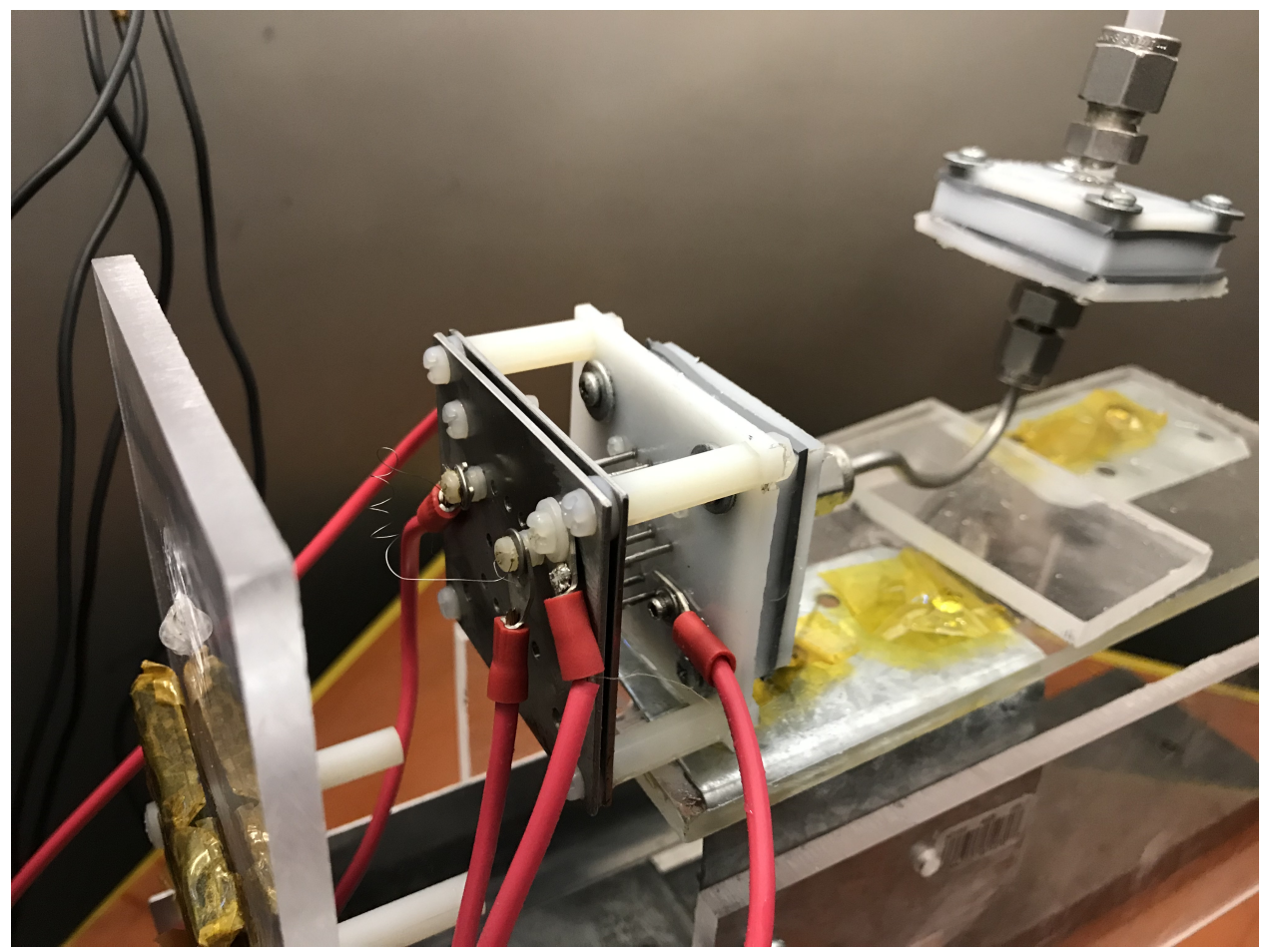

Figure 13: Test Assembly: The thruster is shown having all of the electrical leads connected. The clear propellant collector plate is placed at an inch away from the accelerator grid.

thruster to be able to gather the propellant emitted but not touching the plate such that the filament neutralizer accidentally melts the collector plate. For the purposes of consistency, the collector plate is placed at $1 \mathrm{in}$. from the accelerator grid.

\subsection{Method of Indirect Performance Measurement}

Without the use of direct measuring equipment, the thrust has to be measured indirectly for use in performance calculations. The two key measurements that will be taken are the mass of the propellant, $m_{\mathrm{p}}$, that is gathered on the collection plate and the time, $(t)$, that the thruster is operated. For the testing to develop the performance profile, the time of operation is five minutes for all tests. These measurements gives the mass flow rate of the propellant, $\dot{m}$, as shown in Equation 3.1: 


$$
\dot{m}=\frac{m_{\mathrm{p}}}{t}
$$

The exit velocity of the propellant, $v_{\mathrm{e}}$, is calculated as shown in Equation 3.2 [1]:

$$
v_{\mathrm{e}}=\sqrt{2 \frac{q}{m} V_{\mathrm{a}}}
$$

where $\frac{q}{m}$ is the charge per mass ratio of the propellant and $V_{\mathrm{a}}$ is the voltage applied to the extractor-emitter system and the accelerator grid. Equation 2.4 only calculates the $\max \frac{q}{m}$ available in the propellant using the propellant's chemical and physical properties [12]. In order to find the $\frac{q}{m}$ achieved during experimentation, Equation 2.6 can be used.

However, when using Equation 2.2 to calculate $I_{\mathrm{b}}$, the volumetric flow rate of the propellant that is achieved during the experiment, $Q$, is found in Equation 2.5.

Alternatively, Equation 2.2 and Equation 2.5 are combined into Equation 2.6 to get Equation 3.3:

$$
\frac{q}{m}=\frac{f(\epsilon)}{\rho} \sqrt{\frac{\gamma K}{\epsilon Q}}
$$

where $f(\epsilon)$ is an empirical constant, $\rho$ is the density of the propellant, $\gamma$ is the surface tension of the propellant, $K$ is the conductivity of the propellant, $Q$ is the volumetric flow rate of the propellant, and $\epsilon$ is the relative permittivity of the propellant. The empirical constant, $f(\epsilon)$, that corrects the theoretical calculations to model the actual behavior of electrospray functions. Theoretical calculations operate on a droplet size determined by the propellant properties. The correction provides a more statistically accurate distribution of droplet sizes between the lower bound, which is determined by 
the minimum energy principle, and the upper bound, which is known as the Rayleigh Limit [5], [12]. Having calculated $\frac{q}{m}, v_{\mathrm{e}}$ is calculated using Equation 3.2, and now $T$ and $I_{\mathrm{sp}}$ are calculated using Equation 1.1 and Equation 1.2, respectively.

The $I_{\mathrm{sp}}$ calculation in Equation 1.2 utilizes the assumption that the mass of the emitted propellant is collected perfectly without any losses. In reality, emitted propellant is lost to the grids, and alignment errors can cause emitted propellant to not land on the collector plate. As such, the calculated $I_{\mathrm{sp}}$ is slightly higher than what it actually is. To mitigate this, a high speed camera can be implemented to measure the exhaust velocity of the propellant and use that to calculate the $I_{\mathrm{sp}}$.

The thrust found using Equation 1.1 is the thrust that was actually achieved, which is denoted as $T_{\text {actual }}$. The thrust that is found in Equation 2.1 is the theoretical thrust that can be produces, which is denoted as $T_{\text {theoretical }}$. The thrust efficiency, $\eta$, is then calculated by taking the ratio of $T_{\text {actual }}$ and $T_{\text {theoretical }}$, as shown in Equation 3.4:

$$
\eta=\frac{T_{\text {actual }}}{T_{\text {theoretical }}}
$$

To find the mass of the propellant, the collection plate must be cleaned with alcohol, wiped dry with a Kimwipe to prevent adding dust or lint, and weighed to find the mass of the collection plate before running the test to ensure that the only material on the collection plate is the propellant. After the test, the collection plate with the emitted propellant on it must be weighed again. The mass of the propellant is the difference in the mass of the plate before and after the test, as show in Equation 3.5:

$$
m_{\mathrm{p}}=m_{\mathrm{after}}-m_{\mathrm{plate}}
$$

where $m_{\text {after }}$ is the mass of the collector plate after the test with the propellant and 
$m_{\text {plate }}$ is the mass of the collector plate. Also, to mitigate against any measurement error due to outgassing of the collection plate material, the collection plate should be weighed again after cleaning off the propellant. If any outgassing occurred, the mass of the outgassed collection plate should be used to obtain the mass of the propellant. By using this method, great care must be taken in order to reduce contamination on the collection plate. This means disposable gloves should be worn each time the collection plate is handled. Additionally, the plate should be carried by the edges in order to not disturb any of the propellant that is collected.

It should be noted that using Plexiglas for the collection plate does not allow for high precision scales as it is too heavy for the scale to register the mass. To mitigate this, another material, such as aluminum foil can be used to collect the propellant. Aluminum foil has a low mass, so it can be read on scales with higher precision. However, to have the same visual impact for the students, a dye should be added to the propellant so it can be seen on the aluminum collection plate. This adds complexity and changes the electrical conductivity of the propellant, thus altering the performance. The Plexiglas collection plate is sufficient to gather data while still meeting the objective of creating a visual impact for the students.

\subsection{Method of Proving Electrospray Success}

The first aspect of proving success of the electrospray operation of the thruster is visual confirmation of the expelled propellant. This is accomplished two ways. The initial test is to observe the collection of the propellant on the collection plate. The secondary confirmation test is through visual monitoring to ensure that the main driving force for moving the propellant is from the electrospray process by observing the cone-jet formation on the tips of the emitters. 
The second aspect of proving success is demonstrating that the indirect method of measuring performance characteristics can be repeated. To do this, the voltage applied to the emitter-extractor system is $1000 \mathrm{~V}$. Then, the voltage applied to the accelerator grid is varied, starting at $1000 \mathrm{~V}$ and stepping down by $100 \mathrm{~V}$ for each five minute test, in order to generate a performance profile of the thruster. The HP $20 \mathrm{~V}$ DC power supply for the filament neutralizer is set to $3 \mathrm{~V}$ and $1.5 \mathrm{~A}$ to generate a glow. Each data point consists of three to four tests to demonstrate repeatability and limit system error. Procedures for these tests are fully outlined in Appendix D. This performance profile also serves as a reference as to what data the students should be gathering. Appendix B provides detail as to how the students will perform the lab activity, and Appendix $\mathrm{C}$ provides additional notes to the instructors for running the lab. 
Chapter 4

\section{RESULTS AND DISCUSSION}

The colloid thruster is tested to demonstrate its function as an electrospray thruster. Visual observations are made to confirm that the propellant is being expelled via the electrospray principles. Then, by measuring the amount of propellant expelled and the time of operation, the propellant mass flow rate, thrust, and $\mathrm{I}_{\mathrm{sp}}$ are calculated to form an estimated performance profile. Additional observational analysis is performed on the emission modes and different points of failure.

\subsection{Visual Confirmation}

The first aspect of visual confirmation is observing the collection plate that contains droplets of glycerol. Figure 14 shows a definite deposit of propellant onto the collection plate on the right, showing emission from the colloid thruster when compared to the clean collector plate (left). The propellant is noticeable as it deposits itself as droplets on the plate. To give perspective as to how this plate is oriented in relation to the thruster, the two holes on the bottom are the mounting points to the collection plate stand, which is below the thruster. The propellant has a gradient such that the highest density is in the center, where the thruster is pointed, and then trail off towards the edges of the plate. This type of pattern is expected as alignment errors are present in the emitter array.

Video of the colloid thruster in use proved difficult to capture, as the cone-jet forma-

tions are quite small, and visual access to the chamber is limited. A still photo from a video shows a formation of a cone on one of the emitters, as seen in Figure 15. Cir- 

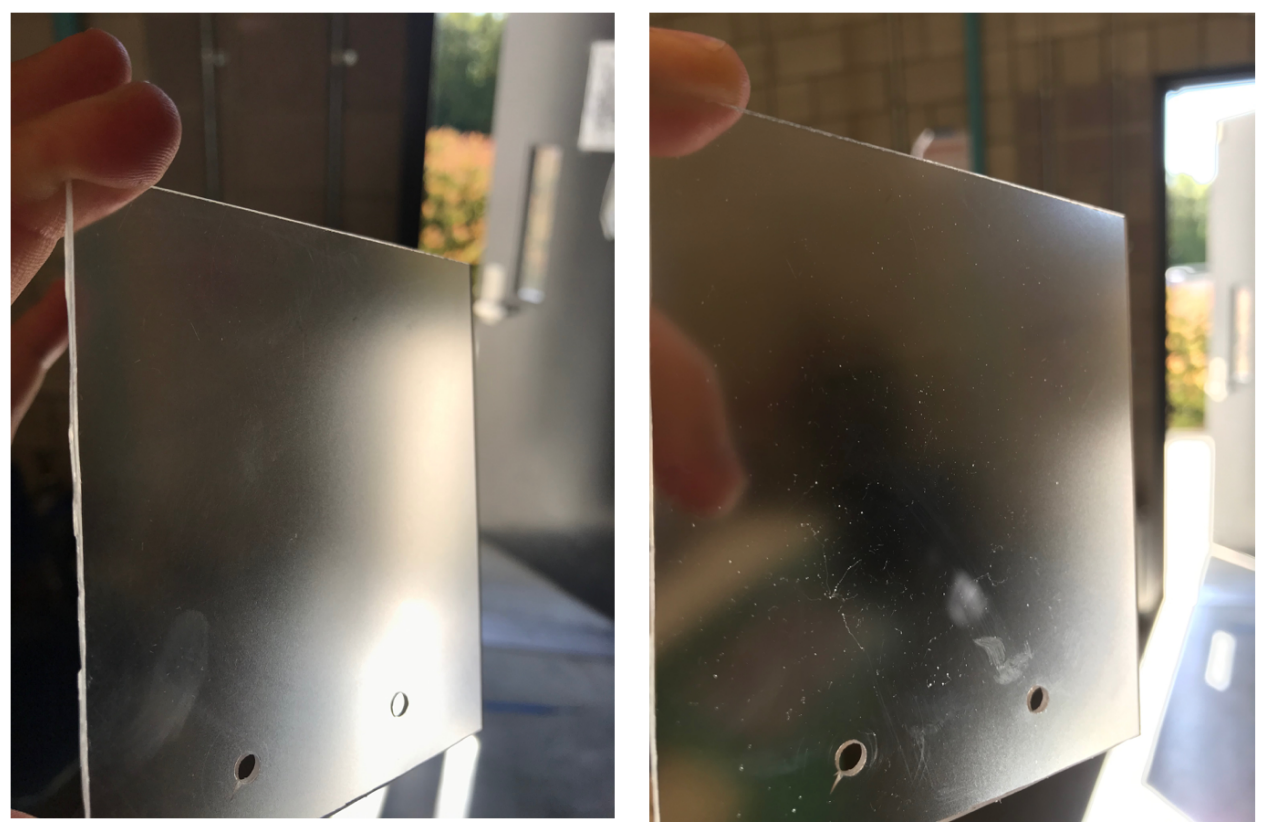

Figure 14: Collection Plate: The glycerol deposits itself as droplets onto the collector plate (right) compared to the clean collector plate (left). This demonstrates that glycerol is being emitted from the thruster.

cled in red is the cone-jet formation. While this does not take on the steady cone-jet formation, as was shown in Figure 3, it does have a bulbous formation that resembles a pulsating emission mode, as shown in Figure 16. The bulbous nature of the cone-jet formation is characteristic of the pulsating emission and transitional pulsating emission modes [11]. These emission modes exist when the electric field is too weak to achieve the desired steady cone-jet emission mode. This indicates that the electric field in this thruster is too weak to create a steady cone-jet emission mode.

Visual inspection also showed that the propellant that is unintentionally leaked out due to the low hydraulic resistance in the emitters drops of the edge of the emitter without passing through the extractor and accelerator grids. Therefore, this propellant does not get deposited on the propellant collection plate and skew the data. Additionally, there is no active propellant feed system in place to forcibly push the propellant out of the emitters. As such, the only force applied to the propellant in order to move the propellant from the emitter to the collection plate is the force due 


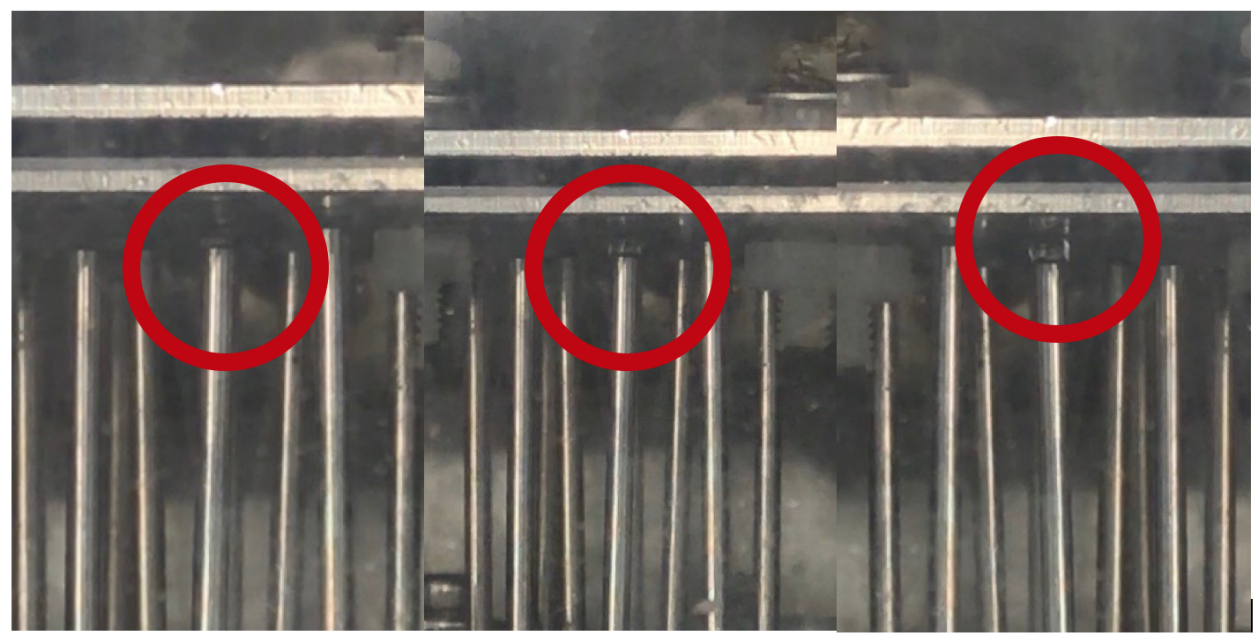

Figure 15: Cone-jet Formation on an Emitter: The bulbous cone that is formed in this picture is similar that of the characteristic bulbous cone of a pulsating emission mode, which is shown in Figure 16.

to the electric field. While the force due to gravity acts on the propellant as well, this force pulls the propellant down rather than pull the propellant horizontally to the collection plate. This along with the bulbous cone formation indicates the movement of propellant is due to the electrospray principle and operates in the pulsating emission mode with the potential to be operating in the transition pulsating emission mode.

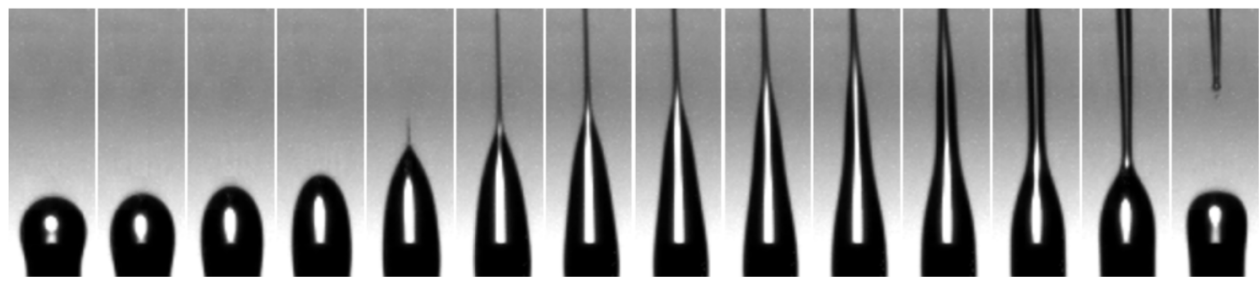

Figure 16: Pulsating Emission Mode [11]: As seen in the left images, the cone formation is quite bulbous. This is the same bulbous formation that is observe at the end of the emitters in this thruster. The middle pictures depict the propellant being drawn off of the cone creating a cone-jet. Then, in the last picture on the right, the jet becomes disconnected and leaving behind just the cone. This intermittent nature of the cone-jet formation is what leads to thruster inefficiency and low performance.

While reviewing the footage, an unexpected failure mode is discovered. As depicted in Figure 17, the cone-jet seems to diverge from the aperture of the extractor grid and 
connect directly to the solid part of the grid. This is most likely due to the alignment errors within the emitters. Given the conductive property of glycerol, the connection that the glycerol makes between the emitter and the extractor grid completes the circuit, which induces a short in the system. Since the electric field is created by applying a voltage across a distance, the short induced by the glycerol eliminates the distance and thus breaks down the electric field. Without the electric field, no propellant is able to be emitted. Since the structural strength of glycerol is quite weak, within 1-2 seconds, the glycerol connection breaks, and emission is then able to resume. This is only observed once during a five-minute test. However, this may be occurring more frequently in emitters that are not easily visible in the chamber. While this outcome is not desired, the system is able to self correct and continue operating with out experimenter interference.

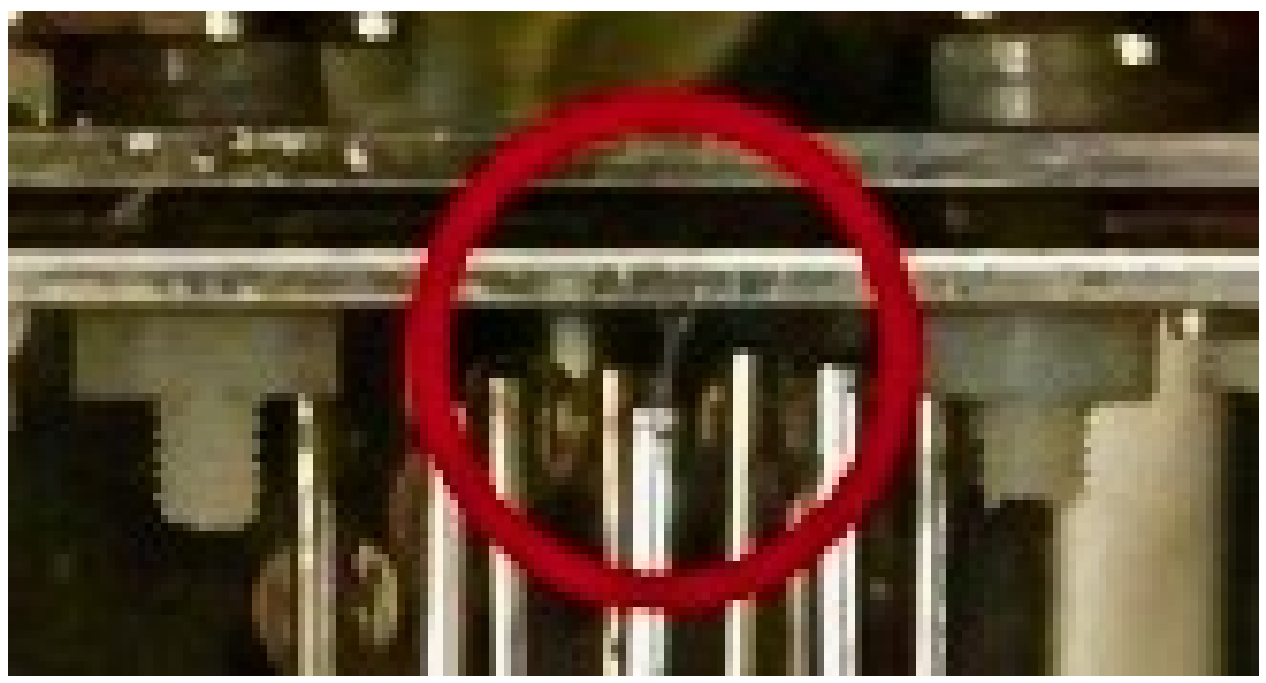

Figure 17: Failure Mode: At this moment, the cone-jet makes direct contact with the solid part of the extractor grid, which causes a short in the system. Since the electric field is made by applying a voltage across a distance, the short eliminates the distance and, in turn, eliminates the electric field. This causes the thruster to stop emitting propellant. However, due to the weak structural property of glycerol, this connection only lasts about 1-2 seconds. 


\subsection{Estimated Performance Profile}

For each accelerator voltage setting, the measured propellant is averaged across the multiple tests performed at their respective accelerator voltage setting in order to reduce the effect of random experimental errors. Using the equations discussed in Section 3.3, the propellant mass flow rate, thrust, and $\mathrm{I}_{\mathrm{sp}}$ of the thruster are calculated, which are shown in Table 2.

Table 2: Estimated Performance Profile of Colloid Thruster: To determine the mass of the propellant expelled, the thruster is tested at each accelerator voltage 3-4 times. The propellant mass collected is averaged with the other propellant measurements for their corresponding accelerator voltage. The errors are found by using the standard deviation of the data and then propagating them through the equations.

\begin{tabular}{|l|l|l|l|l|}
\hline Accelerator & $\begin{array}{l}\text { Mass of } \\
\text { Voltage }(\mathrm{V})\end{array}$ & $\begin{array}{l}\text { Mass Flow } \\
(\mathrm{mg})\end{array}$ & Thrust $(\mu \mathrm{N})$ & $\mathrm{I}_{\mathrm{sp}}(\mathrm{s})$ \\
\hline 1000 & $6.0 \pm 3.6$ & $.025 \pm .013$ & $311.2 \pm 94.6$ & $1270 \pm 40.2$ \\
\hline 900 & $5.6 \pm 3.1$ & $.019 \pm .011$ & $246.8 \pm 64.3$ & $1325 \pm 35.9$ \\
\hline 800 & $3.3 \pm 2.5$ & $.008 \pm .008$ & $129.5 \pm 26.9$ & $1585 \pm 34.4$ \\
\hline 700 & $1.8 \pm 0.6$ & $.006 \pm .002$ & $96.3 \pm 5.2$ & $1684 \pm 9.5$ \\
\hline
\end{tabular}

In Table 2, the lowest accelerator voltage listed is $700 \mathrm{~V}$. Below this voltage, emission does not occur. Given that the electric field is that only force that is used to emit the propellant, the lack of propellant collected suggests that the electric field is too weak to initiate emission when the accelerator voltages is below $700 \mathrm{~V}$. Recall that the accelerator grid's intended purpose is to help focus the beam and increase thrust the thrust produced rather than to help initiate emission. However, since the emission cutoff is determined by the accelerator voltage, this means that the $1000 \mathrm{~V}$ applied to the extractor-accelerator system is too low to create a strong enough electric field to 


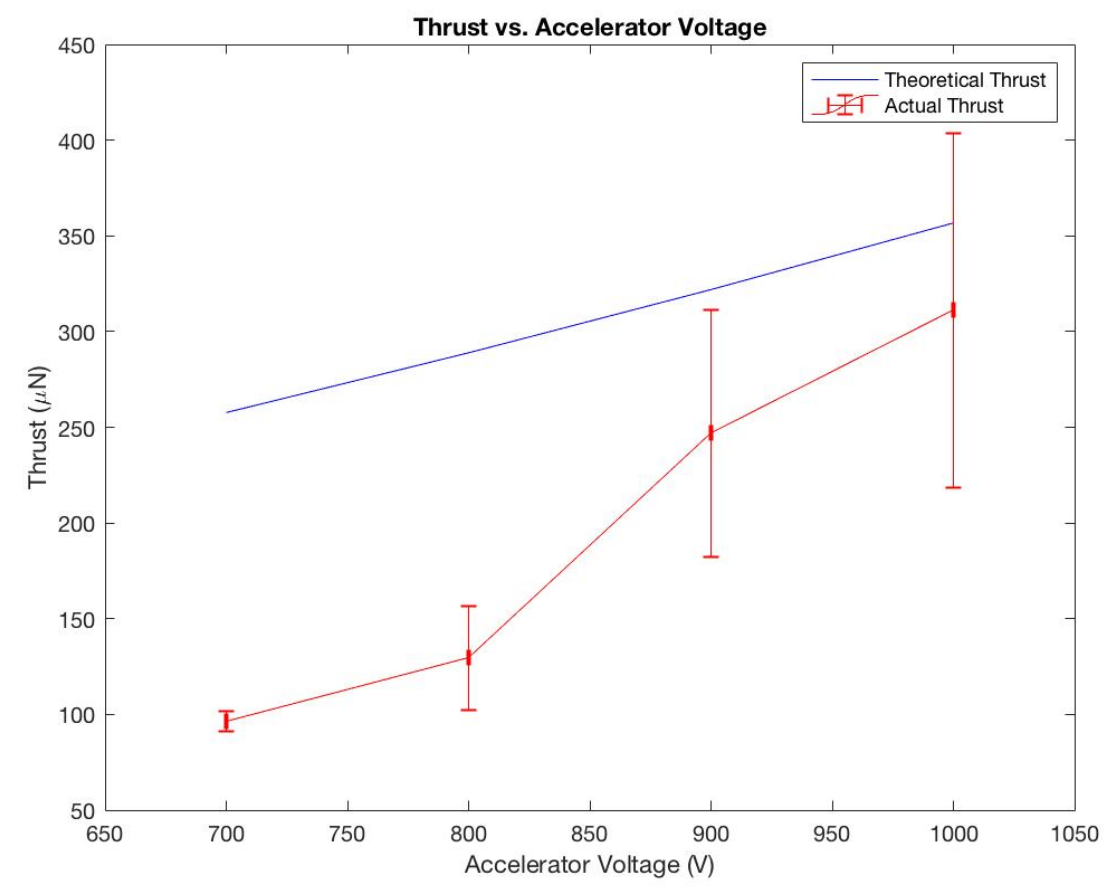

Figure 18: Theoretical Thrust Compared to Estimated Actual Thrust: The actual thrust follows the upward trend of the theoretical thrust produced. The significant jump from $800 \mathrm{~V}$ to $900 \mathrm{~V}$ indicates a higher efficiency. With the stronger electric field at these applied voltages, this jump suggest that the thruster shifted into a transitional pulsating emission mode, which emits propellant at a faster rate. The error bars in this graph are generated from the errors in Table 2.

initiate emission. By understanding that the electric field produced with the assistance of the accelerator grid is on the low end of operation, it makes sense that the cone formations are bulbous and characteristic of pulsating or transitional pulsating emission modes. However, during the laboratory activity, there needs to be a discussion about the fact that the main objectives of the accelerator grid are to help focus the beam and increase the thrust produced.

The comparison between the theoretical thrust and the estimated actual thrust produced, as shown in Figure 18, indicates that the results follow the general trend of the theoretical thrust produced. 
In understanding that the voltage applied is lower than what is required to produce a steady cone-jet emission, the two emission modes most likely being operated in are the pulsating emission and transition pulsating emission modes, as discussed in Section 1.2. The graph in Figure 18 shows a significant jump in performance between $800 \mathrm{~V}$ and $900 \mathrm{~V}$. As transition pulsating emission mode has a higher emission performance than pulsating emission mode [11], this change in performance would indicate that in that region there was a shift from pulsating emission to transition pulsating emission mode. This shift occurs because the next level of acceleration voltage adds enough potential to the electric field to have a stronger pull on the conejet formation. The transitional pulsating emission mode emits propellant at a faster rate than the pulsating emission mode. This increases efficiency as well as the thrust produced, which explains why the thrust in this region is higher and closer to the theoretical thrust produce if it were operating in a steady cone-jet emission mode.

A separate study completed shows the effectiveness of the neutralizer. The thruster ran three times at an accelerator voltage of $800 \mathrm{~V}$ with the neutralizer turned off. The thruster emitted an average of $2.3 \mathrm{mg}$ of propellant. This yields a thrust of 121.6 $\mu \mathrm{N}$ and a specific impulse of $1619 \mathrm{~s}$. This falls inside of the error bounds at the 800 $\mathrm{V}$ range. Therefore, the neutralizer has no significant effect on the flow. However, the neutralizer should still be implemented as it is demonstrates how neutralizers are integrated with electrospray thrusters designed for research and industry.

In this system, the two main contributions to errors are instrumentation errors, which can be directly determined, and system operation errors that cannot be directly determined. The instrumentation error is generated from the scale used to find the mass of the propellant. This error is $\pm .5 \mathrm{mg}$ as the scale's digital readout is precise to $1 \mathrm{mg}$. The system operation errors come from intermittent arcing, the single event upset described above in Section 4.2, and any propellant that may have been moved from 
non-electrospray principles such as outgassing. This error is determined by finding the standard deviation of the propellant mass data. This error is then propagated through the calculations to arrive at the different errors for propellant mass flow rate, thrust, and $\mathrm{I}_{\mathrm{sp}}$.

\subsection{Implementation as a Lab}

In Fall Quarter 2018, the colloid thruster lab was introduced to Cal Poly's AERO 402 Spacecraft Propulsion Lab in order to help teach electric propulsion. This lab was taught by three instructors over four sections of students. Of these four sections, only two sections were able to successfully operate the thruster. One section failed to operate the thruster because the instructor spent time in the beginning trying to teach electrospray theory and have the students take a close in depth look at the thruster. Then, as students take more time to set up than instructors, there was only enough time for one test fire as it takes thirty minutes to fully pump down the chamber, run the thruster, vent, and take mass data. During this test, one of the leads was improperly connected, which was only discovered when the chamber was full pumped down. This left no more time to run it a second time. This was corrected for the next two sections such that the lecture occurred while the chamber was pumping down the first test and the students had the opportunity for a closer inspection of the thruster. This allowed time for two successful tests with enough time for troubleshooting and fixing in the event there were any issues. The last section failed to run the thruster as there were many equipment failures that were out of the instructor's control, such

as the chamber not pumping down low enough and electrical leads falling off of the thruster during the chamber pump down. The failure of these two sections gave great insight as to how best to prepare the instructors and how to manage the time for the lab, which is reflected in Appendix C. 
The data from a successful run of the students yields a thrust performance of 78.5 $\mu \mathrm{N}$ with a specific impulse of $1803 \mathrm{~s}$, where the accelerator voltage was set to $700 \mathrm{~V}$. This data point lies outside of the error bars. However, it should be noted that the thruster was operated for 15 minutes and had no propellant left inside of the thruster cavities. This means that the thruster could have been running for quite some time without any propellant, which would artificially lower the mass flow rate. A lower mass flow rate results in a lower thrust produced, as they are directly proportional to each other, and a higher specific impulse, as they are inversely proportional to each other. Nonetheless, the data yielded a result close to what is expected.

In order to study the pedagogical impact of the lab activity, exam scores of a question relating electric propulsion and electrospray thrusters are compared between a class that did not have the lab activity against the class that did have the lab activity. The class without the lab activity scored an average of $85.2 \%$ with a standard deviation of $6.0 \%$. The class with the lab activity scored an average of $84.9 \%$ with a standard deviation of $5.7 \%$ [13]. While there is no statistical significance to the correlation between this lab and student exam performance, there is still a pedagogical impact. Students reported in an anonymous survey that by interacting with this system, they were better able to understand how the theory of electrospray transfers to an actual system. These students also expressed how the lab activity helped them better understand electric propulsion as a whole. 
Chapter 5

CONCLUSION

In an effort to expand the spacecraft propulsion curriculum, a colloid thruster is constructed for use in the aerospace engineering undergraduate laboratory curriculum at California Polytechnic State University, San Luis Obispo (Cal Poly). The colloid thruster gives students hands on experience with a propulsion system that is quickly growing in popularity. With cost and interactability in mind, the system consists of parts easily attainable and at a low cost. Students operate the thruster using various acceleration voltages, measure how much propellant was expelled, and calculate thrust produced. Tests show the system is operational and able to provide meaningful data that can be used to gain understanding of the system. With the addition of this system, Cal Poly continues its reputation of "Learn by Doing" to give aerospace engineering students a practical understanding and experiential knowledge of complex propulsion systems.

As this system is to be used in an undergraduate laboratory experiment, the colloid thruster that was designed, constructed, and implemented operates with equipment designated for undergraduate use and be low cost to construct, operate, and maintain. The design is driven to provide opportunities for high student interaction and tactile impact with the thruster, while still imitating the types of electrospray thrusters they will in electrospray research and development. Along with this, the thruster also needs to provide meaningful and repeatable data. The full lab activity must be able to be completed within the two hours and fifty minutes that are allotted for a lab activity at Cal Poly. This system is not designed to maximize thrust performance or efficiency. This system will also not be flight qualified. 
In order to be considered a successful demonstration, the colloid thruster needs to be able to emit propellant using electrospray principles and be able to generate meaningful data using the equipment designated for undergraduate use. To demonstrate these principles, an estimated performance profile of mass flow rate, thrust, and $\mathrm{I}_{\mathrm{sp}}$ is successfully generated using the indirect measuring system outlined in Section 3.3, pictures of the collection plate with propellant were taken, and a video showing the operation was taken as well. The thruster is shown to generate between 96-311 $\mu \mathrm{N}$ with an $\mathrm{I}_{\mathrm{sp}}$ ranging from 1270-1684 seconds. This profile can be used as a comparison during lab activities.

The data taken showed operation of the thruster when the accelerator grid was carrying a voltage between $700-1000 \mathrm{~V}$. This indicates that the accelerator grid was adding to the electric field in order to initiate emission. Therefore, the $1000 \mathrm{~V}$ on the emitter-extractor system was not enough to initiate emission. Additionally, the data showed that the system was operating at pulsating emission and transition pulsating emission modes. Visual inspection showed the bulbous cone-jet formation that is consistent to that of pulsating emission mode. Furthermore, the collection plate showed the collection of propellant and its concentration pattern to where the emitters are located.

Having the opportunity to implement the thruster as a lab, valuable insight was gained as to how effective the thruster is as a teaching aid as well as how to best utilize the time allotted for the activity. Students indicated through an anonymous survey that the lab activity helped their understanding of how electrospray thrusters work as well as their understanding of electric propulsion as a whole. Overall, the colloid thruster met the criteria of maintaining Cal Poly's pedagogical philosophy of "Learn by Doing" by providing the opportunity of experiential learning. 


\subsection{Future Work}

The colloid thruster as designed in this thesis has room for continued work to be done. The main areas of advancement lie in manufacturing, hardware implementation, and hardware procurement.

As discussed in Section 3.3, the emitters are hand-soldered, which induces alignment issues. The emitter plate that is meant to hold the emitters could use a redesign to minimize the size of the through holes. This design requires less solder to bind the emitter to the emitter plate. Additionally, a more robust manufacturing procedure or manufacturing jig should be looked into in order to achieve a more precise alignment and stronger solder joints.

The Glassman High Voltage power supply doesn't provide enough power to the emitter-extractor system to initiate emission without the aid of the accelerator grid. The procurement of a power supply to provide higher voltages will create a steady cone-jet emission without the use of an accelerator grid. This way, a more complete performance profile can be established, and further investigation into different emission modes and their effects on thruster performance can be available.

The thruster is designed with the potential of having a nitrogen back-pressure, but it is not implemented as there is not a sensitive enough regulator to control the nitrogen flow. By having a proper pressure regulator, a nitrogen back-pressure can be implemented to demonstrate the operation of an active propellant feed system.

While there is equipment to protect against leaking propellant, it would still be good to look further into reducing the leaking propellant. It appears that most of the leakage is occurring around the emitters. Different methods should be looked at in order to properly seal off the emitters from the propellant cavity. Additionally, a 
method of inducing a higher hydraulic pressure in the emitters should be looked into as well, especially if a nitrogen back pressure is implemented.

In all cases of improvement, testing should be completed to generate a new performance profile to see if and how the performance has changed and make sure all systems are operable. This new performance profile can then provide a new baseline for the students to compare their data against.

With the use of this thruster, students have the opportunity to learn about advanced electric propulsion techniques while being at Cal Poly. May this educational tool serve well to prepare the next generation of Aerospace Engineers. 


\section{BIBLIOGRAPHY}

[1] Session 20: Electrospray Propulsion, MIT OpenCourseWare, Massachusetts Institute of Technology, 2015.

[2] N. Timilsina, "Electrospray Thrusters for Attitude Control of a 1-U CubeSat," Master's thesis, University of California, Irvine, 2014. [Online]. Available: https://escholarship.org/uc/item/3506d0z7.

[3] D. G. Courtney, S. Dandavino, and H. Shea, "Comparing Direct and Indirect Thrust Measurements from Passively Fed Ionic Electrospray Thrusters," Journal of Propulsion and Power, vol. 32, no. 2, 2015, DOI:10.2514/1.B35836.

[4] F. Mier-Hicks and P. C. Lozano, "Electrospray Thrusters as Precise Attitude Control Actuators for Small Satellites," Journal of Guidance, Control, AND Dynamics, vol. 40, no. 3, 2017, DOI:10.2514/1.G000736.

[5] L. F. Velazquez-Garcia, "The Design, Fabrication and Testing of Micro-fabricated Linear and Planar Colloid Thruster Arrays," PhD thesis, Massachusetts Institute of Technology, 2004.

[6] J. A. Nabity, G. Mason, and J. R. Engel, "Studies of MEMS Colloid Thrusters," American Institute of Aeronautics and Astronautics,

[7] F. M. Pranajaya and M. A. Cappelli, "Performance Studies of a Colloid Thruster System," International Electric Propulsion Conference, 2001, IEPC-01-284.

[8] J. K. Ziemer, T. M. Randolph, and G. W. Franklin, "Colloid Micro-Newton Thruster for the Space Technology 7 Mission.," IEEEAC, 2010, ISBN: 978-14244-3888-4/10. 
[9] T. Morris, C. Malardier-Jugroot, and M. Jugroot, "Characterization of electrospray beams for micro-spacecraft electric propulsion applications," Journal of Electrostatics, vol. 71, pp. 931-938, 2013, DOI: http://dx.doi.org/10.1016/j.elstat.2013.07.008.

[10] A. Thuppul, P. L. Wright, and R. E. Wirz, "Lifetime Considerations and Estimation for Electrospray Thrusters," AIAA Propulsion and Energy Forum, 2018, DOI: $10.2514 / 6.2018-4652$.

[11] P. L. Wright, A. Thuppul, and R. E. Wirz, "Life-Limiting Emission Modes for Electrospray Thrusters," AIAA Propulsion and Energy Forum, 2018, DOI: $10.2514 / 6.2018-4726$.

[12] Session 22-23: Cone-jet Electrosprays, or Colloid Thrusters, MIT OpenCourseWare, Massachusetts Institute of Technology, 2015.

[13] A. Greig, A. Powaser, D. Howe, and W. McGehee, "Disparate Electrospray Systems for Undergraduate and Graduate Education," ASEE 2019 Annual Conference, 2019.

[14] V. Hruby, M. Gamero-Castaño, P. Falkos, and S. Shenoy, "Micro Newton Colloid Thruster System Development," International Electric Propulsion Conference, 2001, IEPC-01-281.

[15] B. Gassend, L. F. Velazquez-Garcia, A. I. Akinwande, and M. M. fffd.-S. fffdfffdnchez, "Fabrication of a Fully Integrated Electrospray Array with Applications to Space Propulsion," IEEE, 2008, ISBN:978-1-4244-1793-3/08.

[16] R. C. Schank, "What We Learn When We Learn By Doing," Cogprints, University of Southampton, 1998, Paper ID: 637.

[17] Physical Properties of Glycerine and Its Solutions, Glycerine Producers' Association, New York, 1963.

[18] Electrical breakdown limits for MEMS, URL:http://www2.ece.rochester.edu/courses/ECE234, University of Rochester, 2007. 
[19] M. Gamero-Castaño and V. Hruby, "Characterization of a Colloid Thruster Performing in the micro-Newton Thrust Range," International Electric Propulsion Conference, 2001, IEPC-01-282.

[20] S. Dandavino, C. Ataman, C. N. Ryan, S. C. abd D Courtney, J. P. W. Stark, and H. Shea, "Microfabricated electrospray emitter arrays with integrated extractor and accelerator electrodes for the propulsion of small spacecraft," Journal of Micromechanics and Microengineering, 2014, DOI: 10.1088/0960-1317/24/7/075011. 


\section{APPENDICES}

Appendix A

COLLOID THRUSTER PART DRAWINGS 


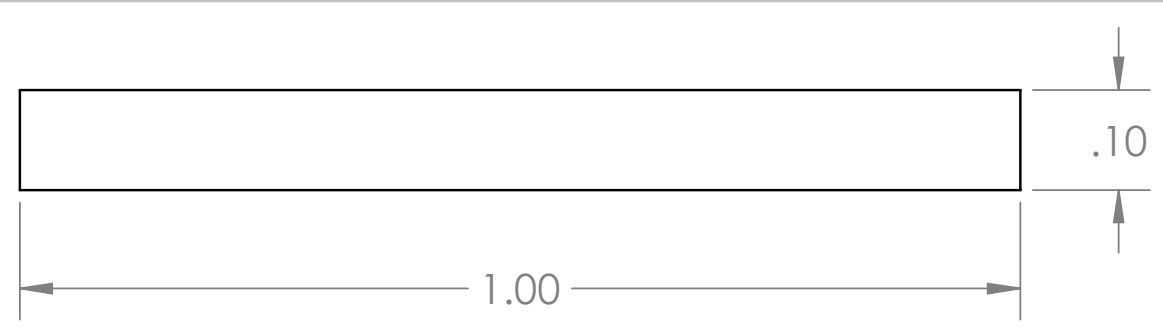

B

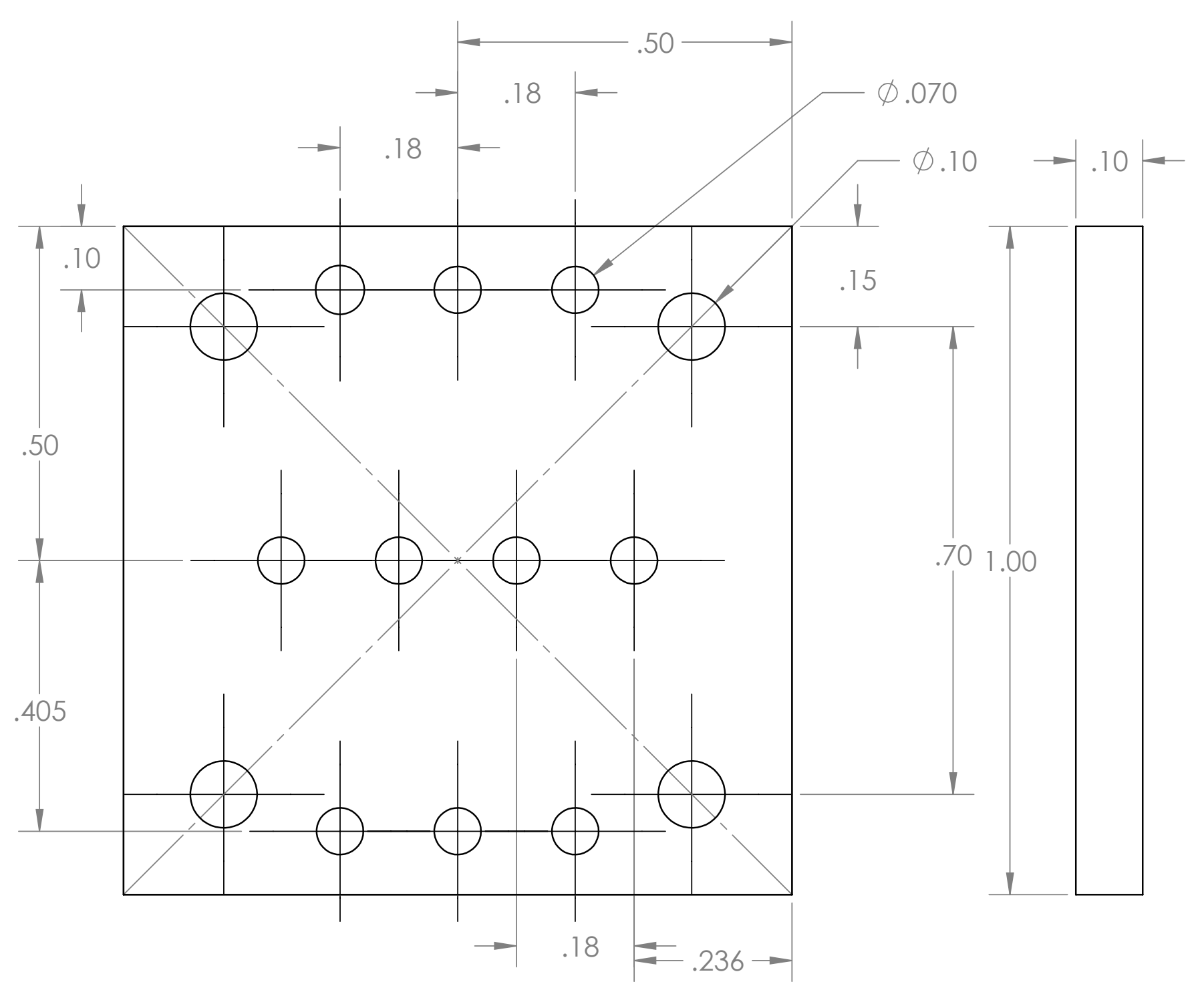

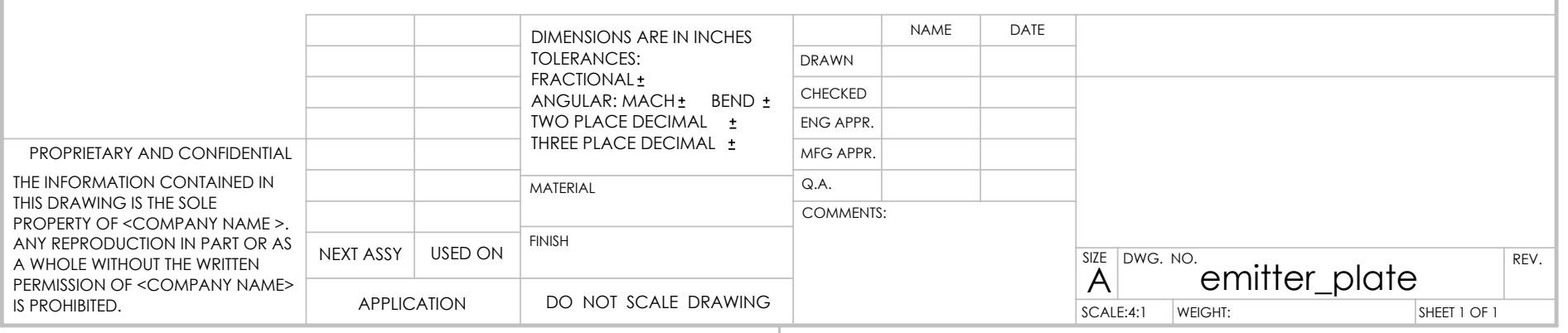




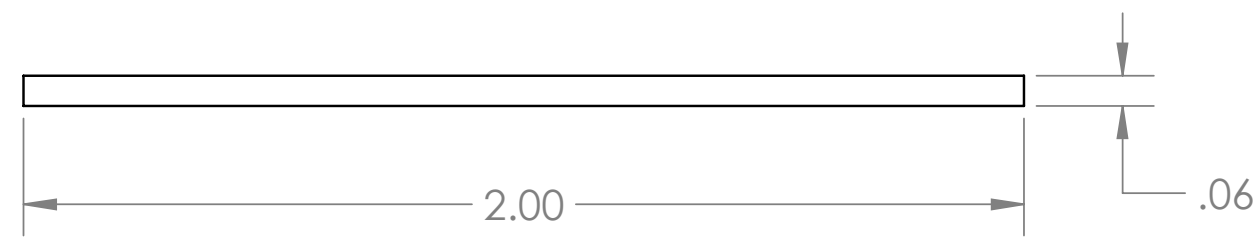

B

A

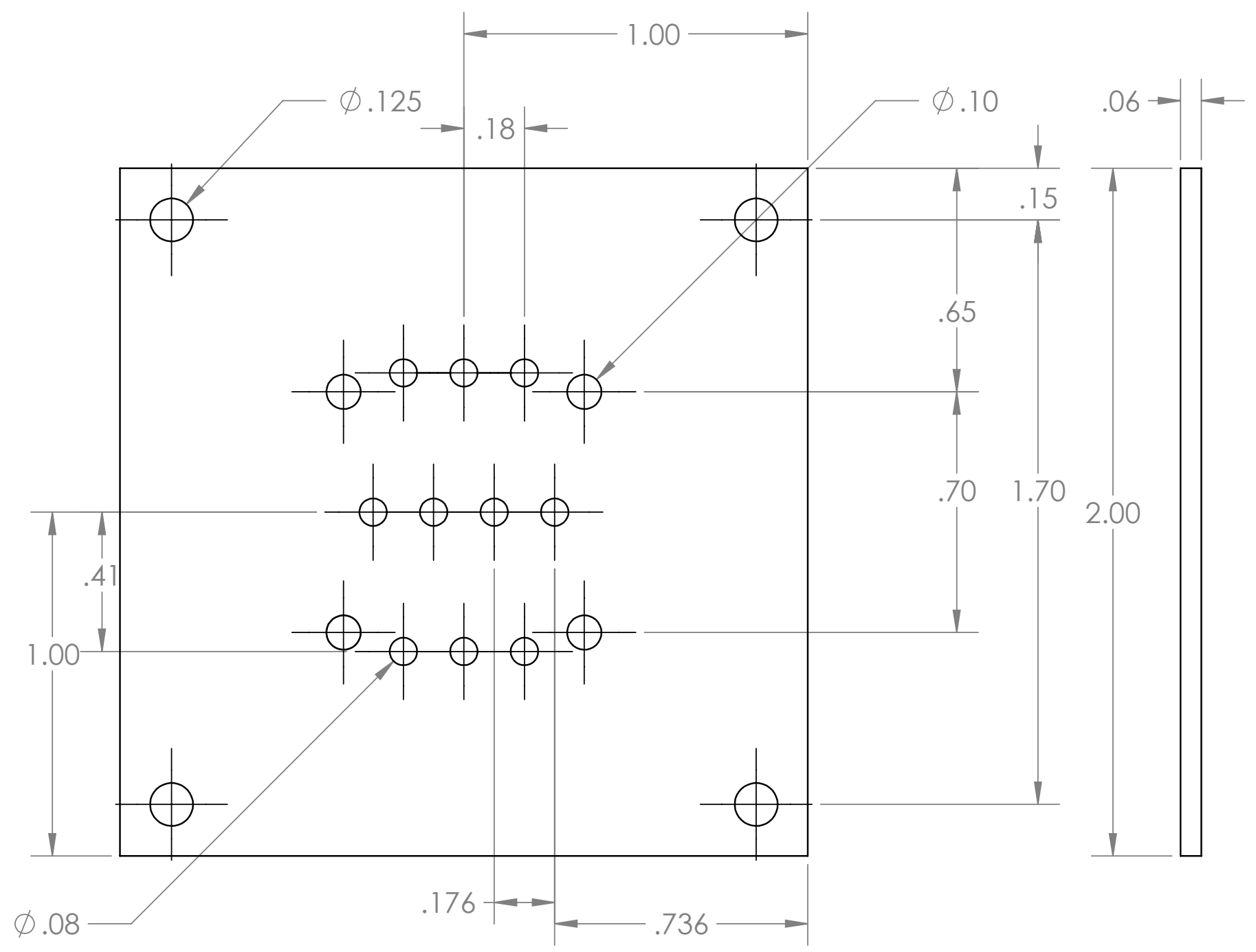

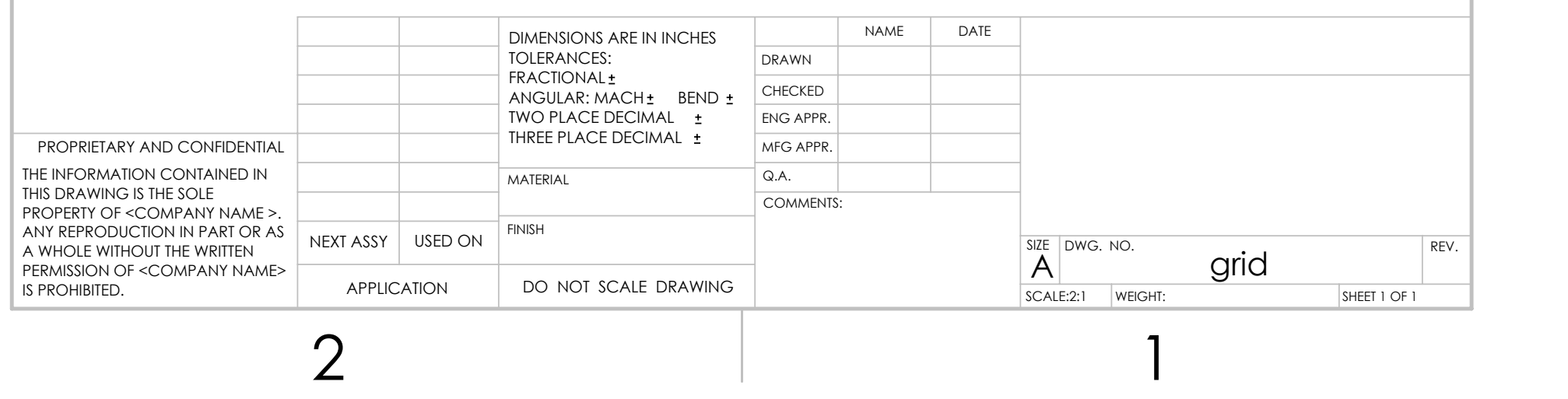



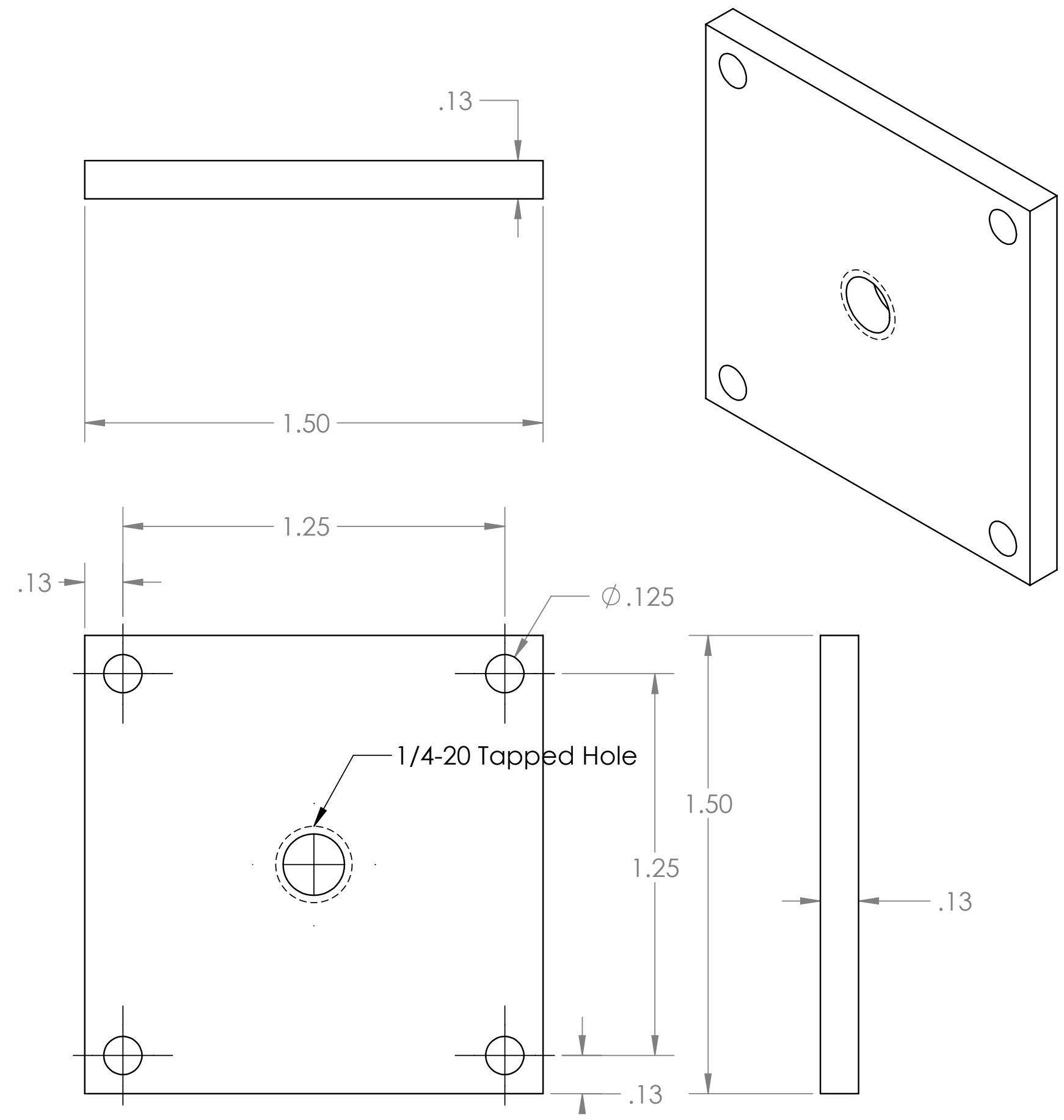

A

PROPRIETARY AND CONFIDENTIAL THE INFORMATION CONTAINED IN THIS DRAWING IS THE SOLE

PROPERTY OF <COMPANY NAME >

ANY REPRODUCTION IN PART OR AS A WHOLE WITHOUT THE WRITTEN

PERMISSION OF <COMPANY NAME> IS PROHIBITED.

\begin{tabular}{l} 
DIMENSIONS ARE IN INCHES \\
TOLERANCES: \\
FRACTIONAL \pm \\
ANGULAR: MACH $\pm \quad$ BEND \pm \\
TWO PLACE DECIMAL $\quad \pm$ \\
THREE PLACE DECIMAL \pm \\
\hline MATERIAL \\
\hline FINISH \\
\hline
\end{tabular}

DO NOT SCALE DRAWING

\begin{tabular}{|l|l|l|} 
& NAME & DATE \\
\hline DRAWN & & \\
\hline CHECKED & & \\
\hline ENG APPR. & & \\
\hline MFG APPR. & & \\
\hline Q.A. & & \\
\hline COMMENTS: & \\
\hline
\end{tabular}

NEXT ASSY USED ON APPLICATION

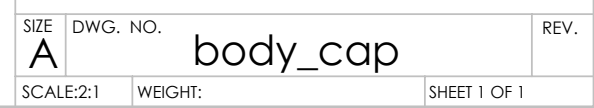

B 
B

1

\section{.25} 1

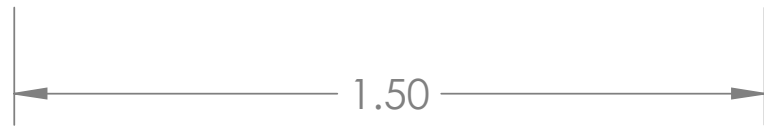

A

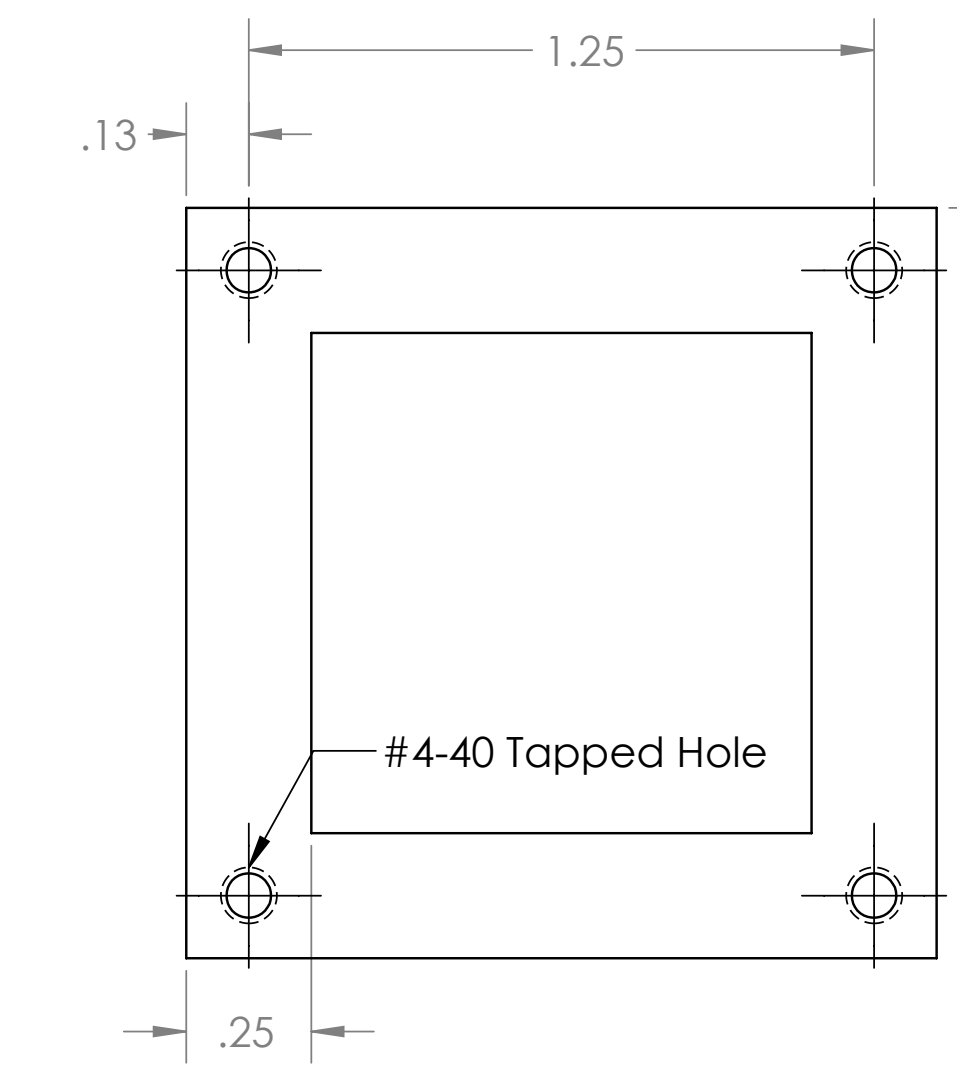

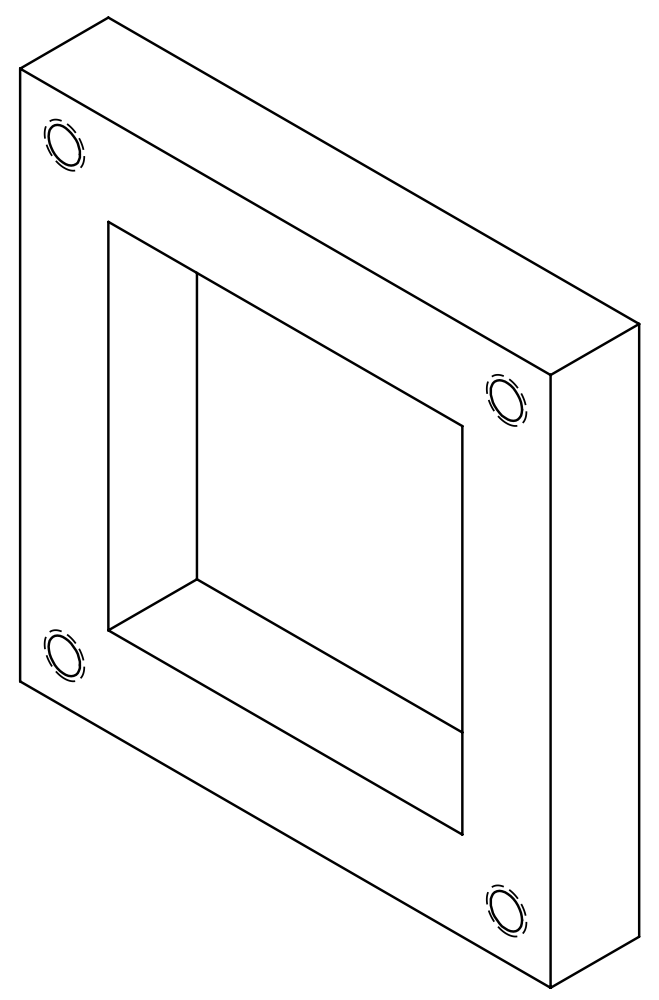

.13

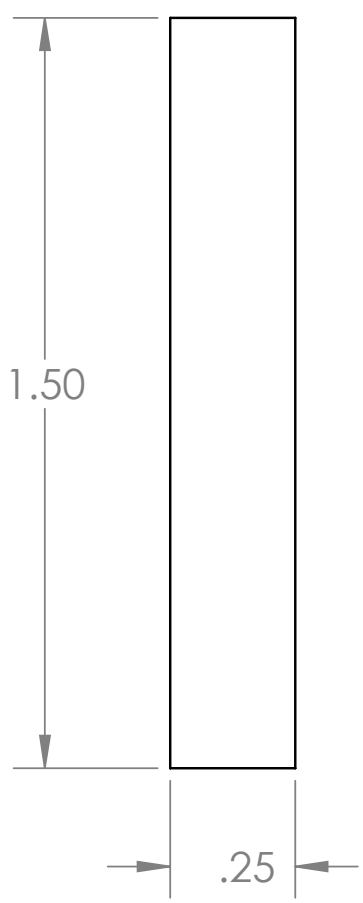

B

A

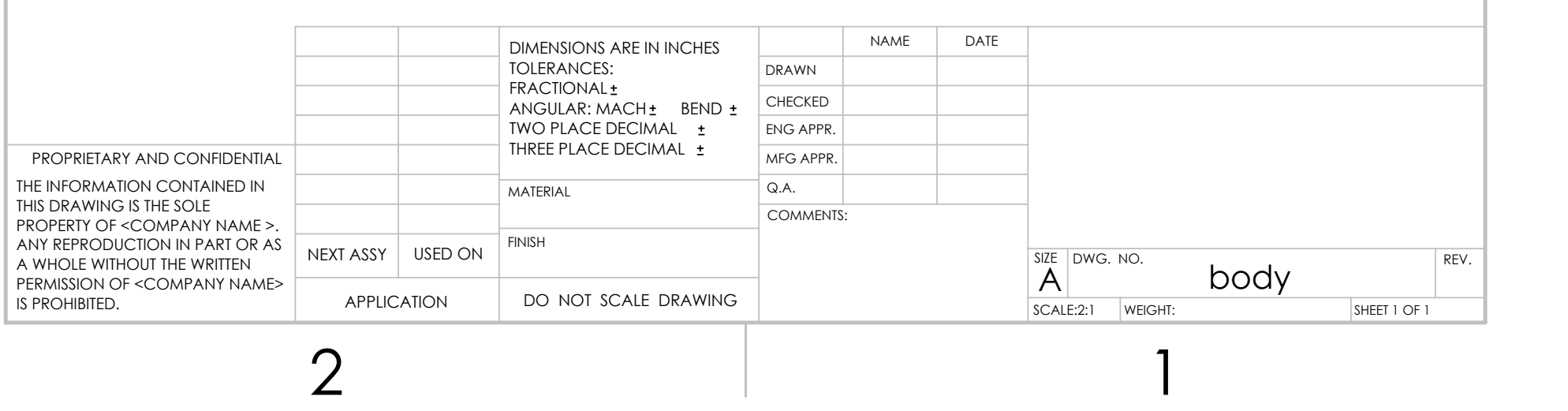



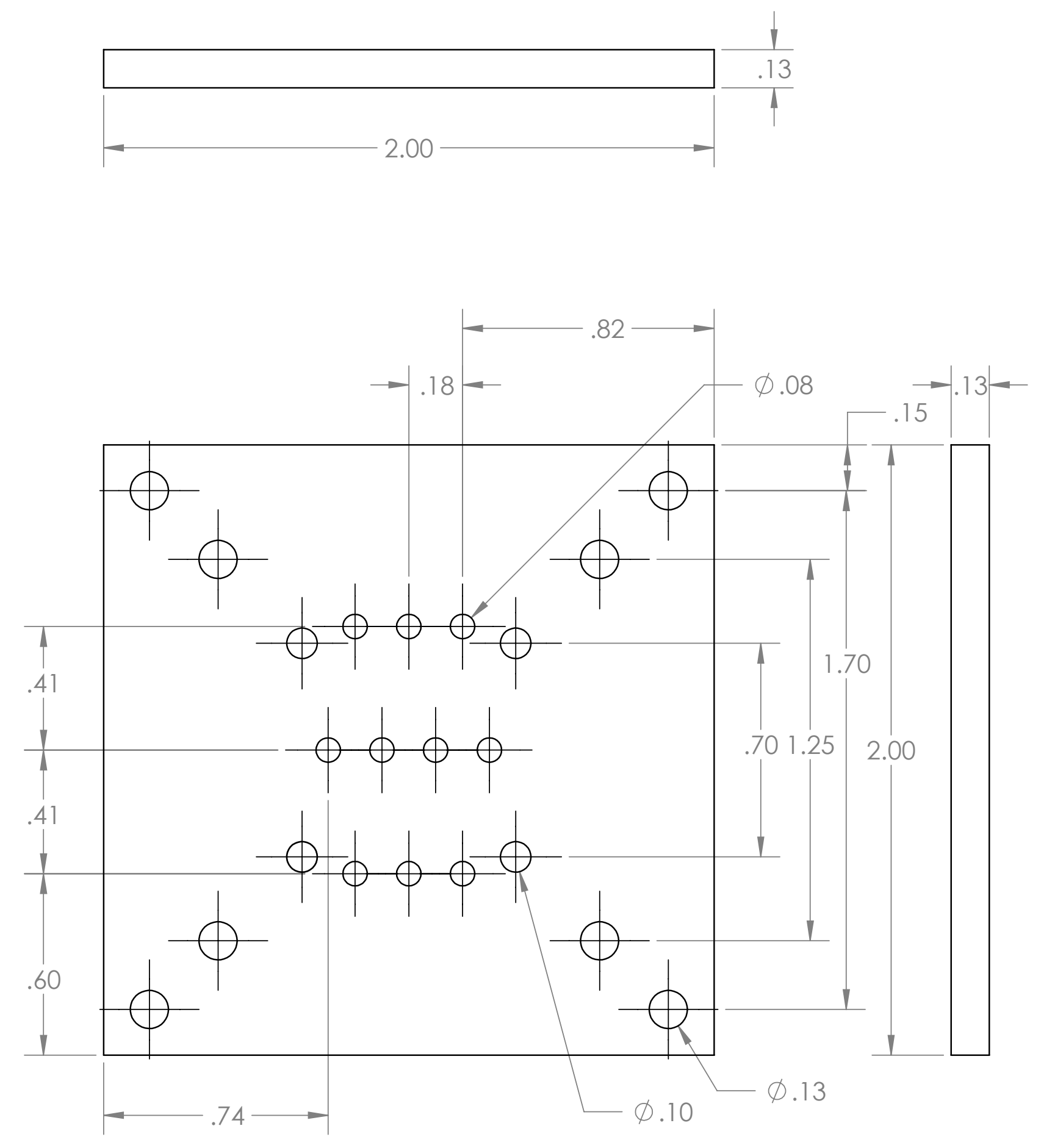

\begin{tabular}{|c|c|c|c|c|c|c|c|c|c|}
\hline \multirow{9}{*}{$\begin{array}{l}\text { PROPRIETARY AND CONFIDENTIAL } \\
\text { THE INFORMATION CONTAINED IN } \\
\text { THIS DRAWING IS THE SOLE } \\
\text { PROPERTY OF <COMPANY NAME >. } \\
\text { ANY REPRODUCTION IN PART OR AS } \\
\text { A WHOLE WITHOUT THE WRITTEN } \\
\text { PERMISSION OF <COMPANY NAME> } \\
\text { IS PROHIBITED. }\end{array}$} & & & \multirow{5}{*}{$\begin{array}{l}\text { DIMENSIONS ARE IN INCHES } \\
\text { TOLERANCES: } \\
\text { FRACTIONAL } \pm \\
\text { ANGULAR: MACH } \pm \text { BEND } \pm \\
\text { TWO PLACE DECIMAL } \\
\text { THREE PLACE DECIMAL } \pm\end{array}$} & \multirow[b]{2}{*}{ DRAWN } & NAME & DATE & & \multirow{2}{*}{+2} & \\
\hline & & & & & & & & & \\
\hline & & & & \multicolumn{3}{|l|}{ CHECKED } & \multirow{7}{*}{\multicolumn{2}{|c|}{\begin{tabular}{c|c} 
SIZE & DWG. No. \\
& emitter_cap
\end{tabular}}} & \\
\hline & & & & \multirow{2}{*}{\multicolumn{3}{|c|}{\begin{tabular}{|l|} 
ENG APPR. \\
MFG APPR.
\end{tabular}}} & & & \\
\hline & & & & & & & & & \\
\hline & & & \multirow[t]{2}{*}{ MATERIAL } & Q.A. & & & & & \\
\hline & & & & \multirow{3}{*}{\multicolumn{3}{|c|}{ COMMENTS: }} & & & \\
\hline & NEXT ASSY & USED ON & FINISH & & & & & & REV. \\
\hline & \multicolumn{2}{|c|}{ APPLICATION } & & & & & & & SHEET I OF I \\
\hline
\end{tabular}




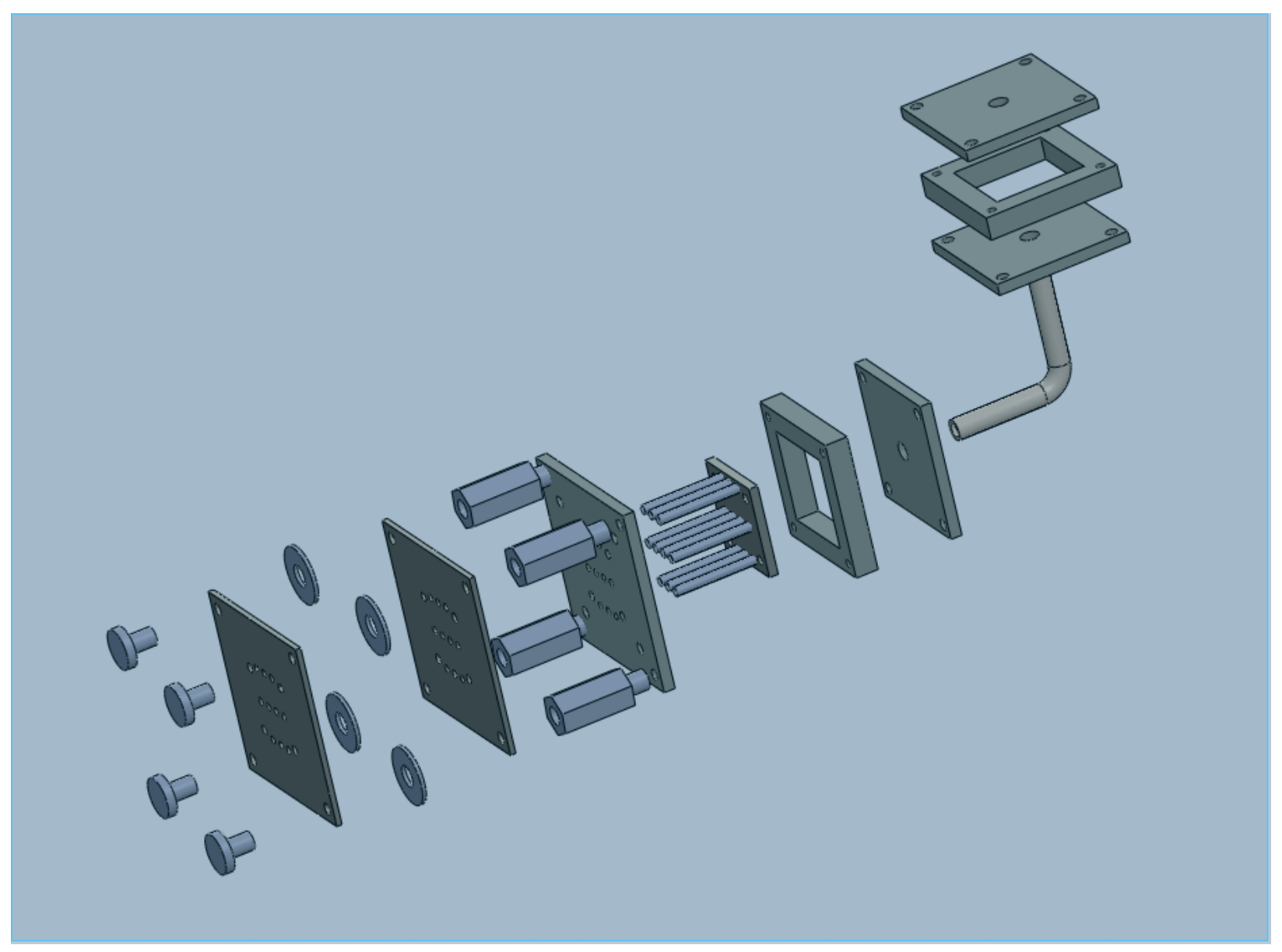


Appendix B

STUDENT LAB DOCUMENT 


\section{AERO402: Spacecraft Propulsion Systems (Fall 2018)}

Electric Propulsion - Colloid Thruster

Week of: 28 Nov 2018

Assessment: Slide Deck

Due: 11:59pm Two Weeks from Lab Session

Online Submission

Maximum score: 40 points

The laboratory session will be completed as one groups of about 20 people. Everyone is expected to stay for the entire 2 hours and 50 minutes. Only about 10 people can be involved in a single firing, so utilize the extra time to start writing the laboratory assessment.

The slide deck should be completed in groups of 3-4. You may choose your own groups, but must be with people in the same lab session.

Meet in Environments Lab (ENG3-137) at your assigned time. Wear closed toed shoes, no loose clothing. Bring paper, pencil, calculator, and print off or have quick access to a digital version of the Electric Propulsion Lab Outline and Colloid Thruster Apparatus.

\section{Additional Safety for this lab}

1) Do not touch the vacuum chamber when the Colloid Thruster is running

2) If arcing outside the chamber occurs (loud cracking sounds or visible arcs) turn the Colloid Thruster off immediately

3) The mechanical pump used on the vacuum chamber uses oil, and small leaks may result in the ground between the chamber and the wall being very slippery. Only enter this area if you are required too and take care if you do. 


\section{OBJECTIVE:}

The objective for this lab activity is to set-up an experiment to quantitatively measure the thrust produced by the colloid thruster.

\section{BACKGROUND:}

A colloid thruster is a type of electrospray thruster that uses electrostatic forces to accelerate liquid propellants, such as formamide or glycerol, to generate thrust. While these thrusters were first pioneered in the '60s and '70s as an alternative to ion thrusters, they required $\sim 10 \mathrm{kV}$ to run to produce $\mu \mathrm{N}$ levels of thrust. With the advancement of technology, colloid thrusters can now run on the order of 1-5 kV with the same performance. This, along with the growing popularity of CubeSats, have caused colloid thrusters and other electrosprays to be looked at again more in depth. Currently, the first and only flight model was developed and manufactured by Busek and JPL and is flying on the LISA Pathfinder mission.

\section{ELECTROSPRAY OPERATION:}

The basic premise of a colloid thruster is illustrated in Fig. 1. A strong electric potential is applied between an extractor grid and the end of an emitter tip containing a liquid propellant. Taylor cones form, concentrating the electric field strength near the cone tip, and, at sufficient strength, charged droplets will be extracted from the emitter tip. The charged droplets are accelerated by the electrostatic field. Typically, there is also an accelerator grid after the extractor grid, as demonstrated in Fig. 1.

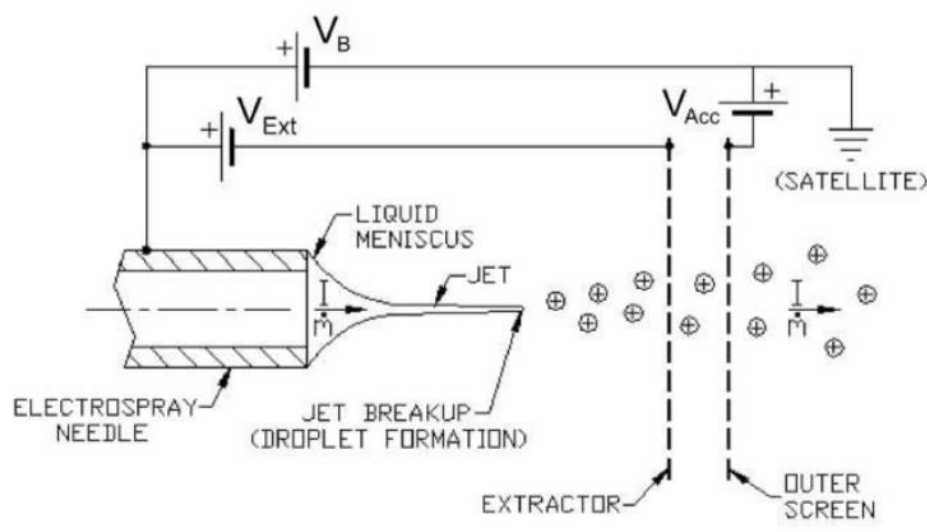

Fig. 1 The Basic Electrospray Configuration

The fundamental aspect of the extraction of the droplets comes from the Taylor Cone structure of an electrified liquid. As seen in Fig. 2, a Taylor Cone is where the liquid is extended beyond the tip of the emitter and forms a cone like shape. The Taylor Cone is an idealized occurrence when the electric field becomes infinitely large at the tip while becoming infinitesimally small in physical size. One of the assumptions for this situation

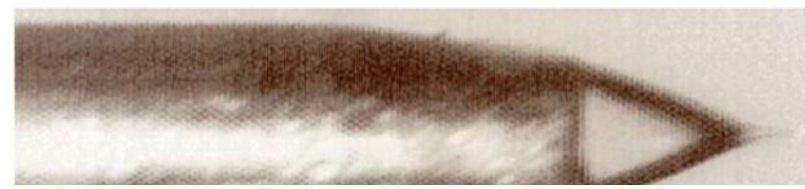

Fig. 2 Cone-jet Formation 
is that there is no flow. This assumption falls apart considering that flow is required in order to produce thrust. Now that the cone is no longer the idealized Taylor Cone, this extraction formation is now referred to as the "cone-jet" mode. Nonetheless, the terms "Taylor Cone" and "cone-jet" are often used interchangeably.

There are three main emitter types to choose from when it comes to designing the emitter-extractor system as seen in Fig. 3. An internal capillary emitter draws propellant from a reservoir and passively moves up the emitter due to capillary forces. The emitter in Fig. 2 above is an internal capillary emitter. An externally wetted emitter pulls propellant over the emitter to the tip and then expelled. A bulk porous is a blend between the other two emitters. The propellant is drawn up through capillary action, secreted to cover the emitter, and then expelled at the tip.

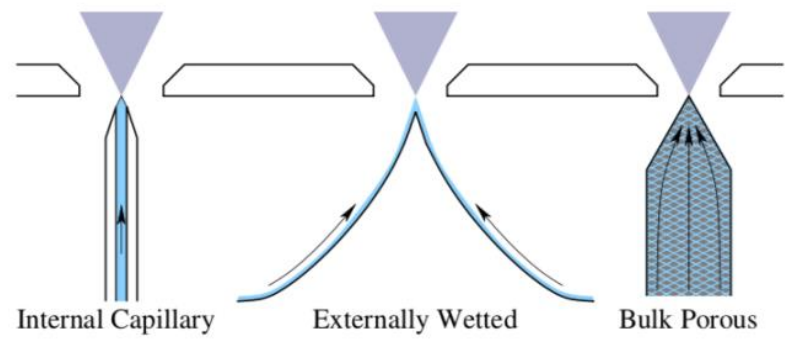

Fig. 3 Three Types of Emitters

With the development of MEMS, a specialized micro-fabrication processes, externally wetted is becoming the most preferred method of emitting the propellant. The bulk process of manufacturing the emitters is through chemical etching. This allows a controlled and uniform method of carving out microscopic entities of the emitter. The process and externally wetted emitters also allow different geometries to be designed to help maximize performance. Along with these benefits, MEMS can optimally integrate the extractor grid and the emitter array.

\section{COLLOID THRUSTER LAB DESIGN:}

While industry utilizes MEMS and works on innovation towards building microscopic components, the design for the lab based colloid thrusters is driven by scaling up what industry produces. A typical colloid thruster produced in industry contains components on the order of $\mu \mathrm{m}$, a propellant pressurant, and bipolar emission to create a quasineutral flow. The colloid thruster for lab contains components on the order of $\mathrm{mm}$ and $\mathrm{cm}$, no propellant pressurant, and a positive bias emission which requires the use of a filament neutralizer to detach the flow. By not having a propellant pressurant, the internal capillary emitters rely on capillary action to keep the emitters full of propellant. The two-propellant cavity design aids in this as the propellant remains equally distributed among the emitters and the propellant is expelled. Additionally, these components were machined in house and the emitters were soldered together manually. 
4) Place drip tray, collection plate stand, and thruster stand in chamber in that order (see Fig. 4)

5) Place electrical leads on Colloid Thruster

a. See Colloid Thruster Apparatus for details

6) Fill thruster with $6 \mathrm{~mL}$ of glycerol via the propellant reservoir

a. Screw plug into propellant reservoir when finished

7) Place thruster on stand with the collection plate approx. $2.5 \mathrm{~cm}$ from thruster

a. Be sure not to bring the collection plate in contact with the neutralizer

8) Place contaminate containment box over the system

9) Place lid on the vacuum chamber, secure lid to chamber, close vent, and turn on pump (see Fig. 5)

10)Wait until the chamber reaches $\sim 60$ mTorr

11)Turn on Glassman A for the emitters and set to $1000 \mathrm{~V}$

12)Turn on Glassman B for the acceleration grid and set between 700-1000 V

13)Turn on neutralizer and set to $3 \mathrm{~V}$ and $1.5 \mathrm{~A}$

14)Let run for $5-7 \mathrm{~min}$

15)Turn off neutralizer, Glassman B, and Glassman A power supplies

16)Turn off pump and vent chamber

17)Remove lid and contaminate containment box

18)Carefully remove collection plate and obtain mass

19)Clean collection plate and take mass again to observe if outgassing occurred

a. If outgassing of the plate occurred, use mass obtained in Step 19 as the mass of the plate

20)Remove thruster

a. If running another test: refill thruster, check all connections, clean up any leaked propellant, replace collection plate, and run again

21)Disconnect all lead from thruster and remove thruster stand, collection plate stand, and drip tray

22)Clean up any residual glycerol if any leaked during the test

23)Rinse emitters and grids of the thruster with alcohol and let dry 


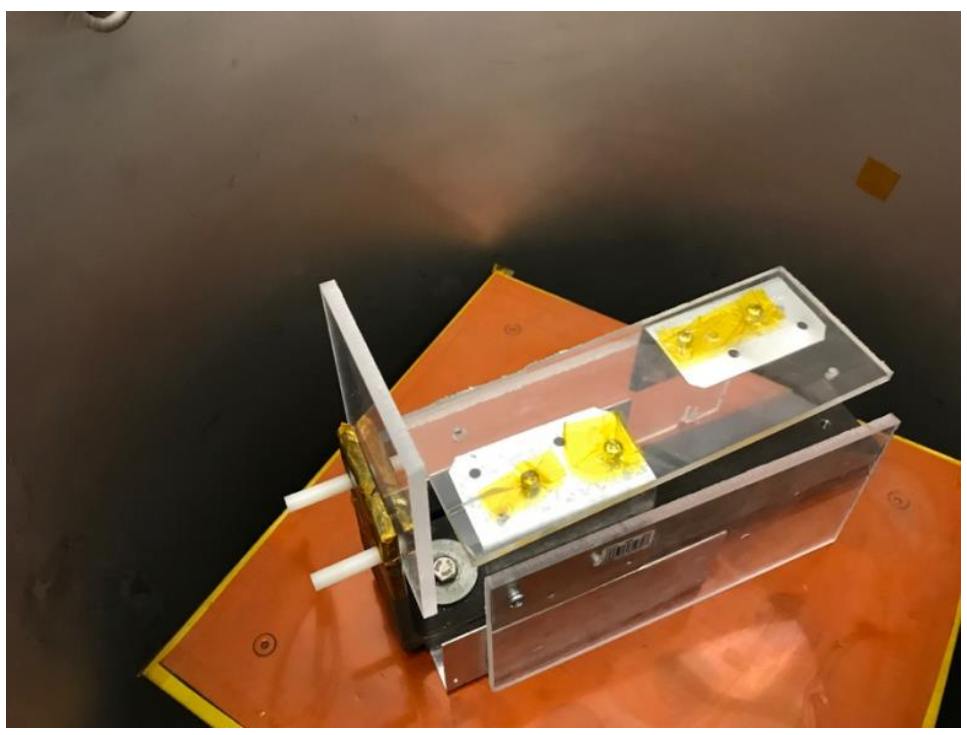

Fig. 4 Stand Set Up

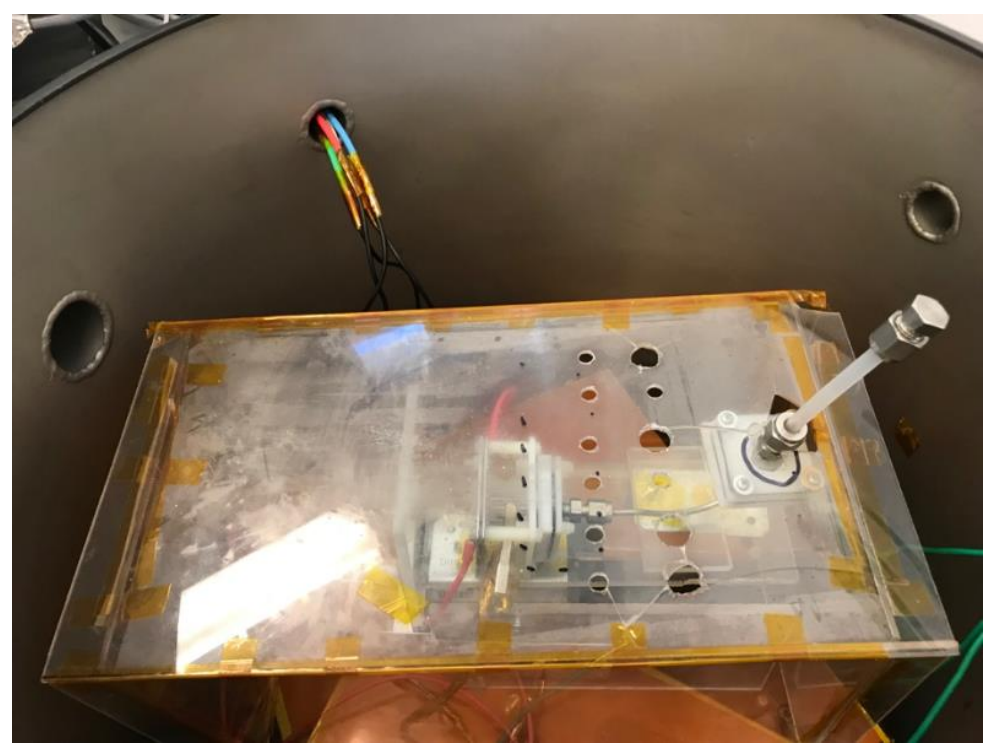

Fig. 5 Complete Set Up 
KEY RESULTS AND DISCUSSION:

1) Estimate colloid thruster performance
a. Estimate thrust produced
b. Estimate thruster efficiency
c. Estimate $I_{\mathrm{sp}}$

2) Discuss the spray pattern on the collection plate. Does it make sense? Why or why not?

3) What can be done to improve thruster efficiency?

4) What additional steps can be taken to improve the testing of the thruster performance?

5) Give some examples of when a colloid thruster can be used in flight.

\section{ASSESSMENT:}

In groups of 3-4 prepare a slide deck outlining your experiment. The following items should be included as a minimum:

- A description of a colloid thruster and how they produce thrust

- An outline of your experimental procedure

- Key results and discussion

\section{MARKING SCHEME:}

Background (Electrospray, thrust production, etc)

(10 points)

Outline of experiment procedure

(10 points)

Key results and thruster performance estimates

(15 points)

Formatting, language, and layout

(5 points) 
Appendix C

INSTRUCTOR LAB DOCUMENT 


\section{AERO402: Spacecraft Propulsion Systems (Fall 2018)}

Electric Propulsion - Colloid Thruster

Week of: 28 Nov 2018

Assessment: Slide Deck

Due: 11:59pm Two Weeks from Lab Session

Online Submission

Maximum score: 40 points

The laboratory session will be completed as one groups of about 20 people. Everyone is expected to stay for the entire 2 hours and 50 minutes. Only about 10 people can be involved in a single firing, so utilize the extra time to start writing the laboratory assessment.

The slide deck should be completed in groups of 3-4. You may choose your own groups, but must be with people in the same lab session.

Meet in Environments Lab (ENG3-137) at your assigned time. Wear closed toed shoes, no loose clothing. Bring paper, pencil, calculator, and print off or have quick access to a digital version of the Electric Propulsion Lab Outline and Colloid Thruster Apparatus.

\section{Additional Safety for this lab}

1) Do not touch the vacuum chamber when the Colloid Thruster is running

2) If arcing outside the chamber occurs (loud cracking sounds or visible arcs) turn the Colloid Thruster off immediately

3) The mechanical pump used on the vacuum chamber uses oil, and small leaks may result in the ground between the chamber and the wall being very slippery. Only enter this area if you are required too and take care if you do. 


\section{OBJECTIVE:}

The objective for this lab activity is to set-up an experiment to quantitatively measure the thrust produced by the colloid thruster.

\section{BACKGROUND:}

Instructor's Note: In order to have enough time for multiple runs and margin for troubleshooting, the background lecture should be done while the chamber is pumping down for the first run.

A colloid thruster is a type of electrospray thruster that uses electrostatic forces to accelerate liquid propellants, such as formamide or glycerol, to generate thrust. While these thrusters were first pioneered in the ' 60 s and '70s as an alternative to ion thrusters, they required $\sim 10 \mathrm{kV}$ to run to produce $\mu \mathrm{N}$ levels of thrust. With the advancement of technology, colloid thrusters can now run on the order of 1-5 kV with the same performance. This, along with the growing popularity of CubeSats, have caused colloid thrusters and other electrosprays to be looked at again more in depth. Currently, the first and only flight model was developed and manufactured by Busek and JPL and is flying on the LISA Pathfinder mission.

\section{ELECTROSPRAY OPERATION:}

The basic premise of a colloid thruster is illustrated in Fig. 1. A strong electric potential is applied between an extractor grid and the end of an emitter tip containing a liquid propellant. Taylor cones form, concentrating the electric field strength near the cone tip, and, at sufficient strength, charged droplets will be extracted from the emitter tip. The charged droplets are accelerated by the electrostatic field. Typically, there is also an accelerator grid after the extractor grid, as demonstrated in Fig. 1.

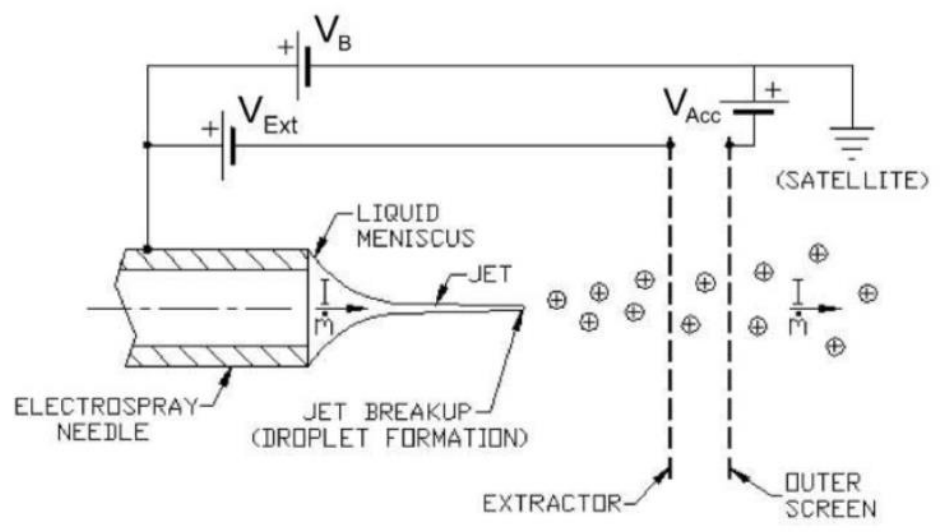

Fig. 1 The Basic Electrospray Configuration

The fundamental aspect of the extraction of the droplets comes from the Taylor Cone structure of an electrified liquid. As seen in Fig. 2, a Taylor Cone is where the liquid is

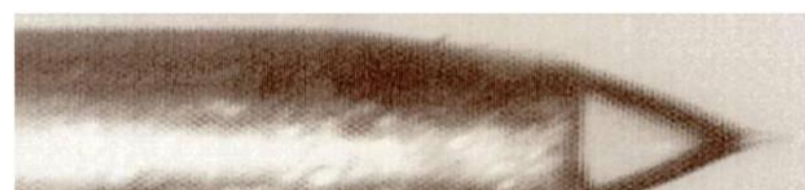

Fig. 2 Cone-jet Formation 
extended beyond the tip of the emitter and forms a cone like shape. The Taylor Cone is an idealized occurrence when the electric field becomes infinitely large at the tip while becoming infinitesimally small in physical size. One of the assumptions for this situation is that there is no flow. This assumption falls apart considering that flow is required in order to produce thrust. Now that the cone is no longer the idealized Taylor Cone, this extraction formation is now referred to as the "cone-jet" mode. Nonetheless, the terms "Taylor Cone" and "cone-jet" are often used interchangeably.

There are three main emitter types to choose from when it comes to designing the emitter-extractor system as seen in Fig. 3. An internal capillary emitter draws propellant from a reservoir and passively moves up the emitter due to capillary forces. The emitter in Fig. 2 above is an internal capillary emitter. An externally wetted emitter pulls propellant over the emitter to the tip and then expelled. A bulk porous is a blend between the other two emitters. The propellant is drawn up through capillary action, secreted to cover the emitter, and then expelled at the tip.

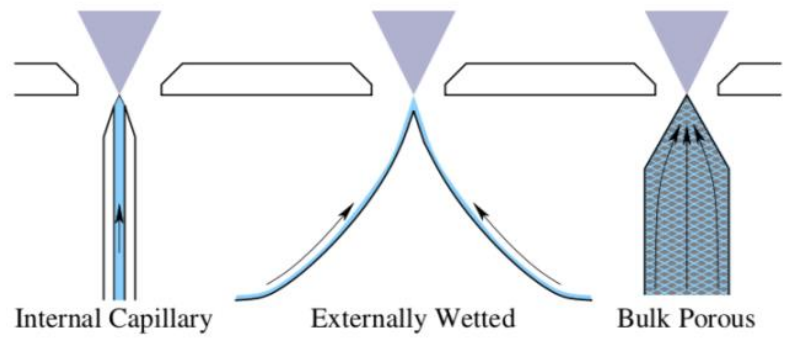

Fig. 3 Three Types of Emitters

With the development of MEMS, a specialized micro-fabrication processes, externally wetted is becoming the most preferred method of emitting the propellant. The bulk process of manufacturing the emitters is through chemical etching. This allows a controlled and uniform method of carving out microscopic entities of the emitter. The process and externally wetted emitters also allow different geometries to be designed to help maximize performance. Along with these benefits, MEMS can optimally integrate the extractor grid and the emitter array.

\section{COLLOID THRUSTER LAB DESIGN:}

While industry utilizes MEMS and works on innovation towards building microscopic components, the design for the lab based colloid thrusters is driven by scaling up what industry produces. A typical colloid thruster produced in industry contains components on the order of $\mu \mathrm{m}$, a propellant pressurant, and bipolar emission to create a quasineutral flow. The colloid thruster for lab contains components on the order of $\mathrm{mm}$ and $\mathrm{cm}$, no propellant pressurant, and a positive bias emission which requires the use of a filament neutralizer to detach the flow. By not having a propellant pressurant, the internal capillary emitters rely on capillary action to keep the emitters full of propellant. The two-propellant cavity design aids in this as the propellant remains equally distributed among the emitters and the propellant is expelled. Additionally, these components were machined in house and the emitters were soldered together manually. 
LAB PROCEDURE:

1) Rinse off grids and emitters with alcohol and let dry

a. Attach filament neutralizer if not already attached

2) Clean off collection plate and obtain and record the mass of the collection plate

3) Secure collection plate to stand with provided nylon screws

4) Place drip tray, collection plate stand, and thruster stand in chamber in that order (see Fig. 4)

5) Place electrical leads on Colloid Thruster

a. See Colloid Thruster Apparatus for details

6) Fill thruster with $6 \mathrm{~mL}$ of glycerol via the propellant reservoir

a. Screw plug into propellant reservoir when finished

7) Place thruster on stand with the collection plate approx. $2.5 \mathrm{~cm}$ from thruster

a. Be sure not to bring the collection plate in contact with the neutralizer

Instructor's Note: It would be time advantageous to have steps 1-7 completed before the first run. Let the students see the set up and point to all of the components.

8) Place contaminate containment box over the system

9) Place lid on the vacuum chamber, secure lid to chamber, close vent, and turn on pump (see Fig. 5)

10)Wait until the chamber reaches $\sim 60$ mTorr

Instructor's Note: The chamber can be at $\sim 90 \mathrm{mTorr}$ and operate fine. There just might be a chance of some arcing.

11)Turn on Glassman A for the emitters and set to $1000 \mathrm{~V}$

12)Turn on Glassman $B$ for the acceleration grid and set between 700-1000 V

13)Turn on neutralizer and set to $3 \mathrm{~V}$ and $1.5 \mathrm{~A}$

Instructor's Note: Remember that no one is allowed to touch the chamber or any of its parts while high voltage power supply is on to prevent any electrical shock.

14)Let run for $5-7 \mathrm{~min}$

15)Turn off neutralizer, Glassman B, and Glassman A power supplies

16)Turn off pump and vent chamber

17)Remove lid and contaminate containment box

18)Carefully remove collection plate and obtain mass

19)Clean collection plate and take mass again to observe if outgassing occurred

a. If outgassing of the plate occurred, use mass obtained in Step 19 as the mass of the plate

20)Remove thruster

a. If running another test: refill thruster, check all connections, clean up any leaked propellant, replace collection plate, and run again

21)Disconnect all lead from thruster and remove thruster stand, collection plate stand, and drip tray

22)Clean up any residual glycerol if any leaked during the test

23)Rinse emitters and grids of the thruster with alcohol and let dry 


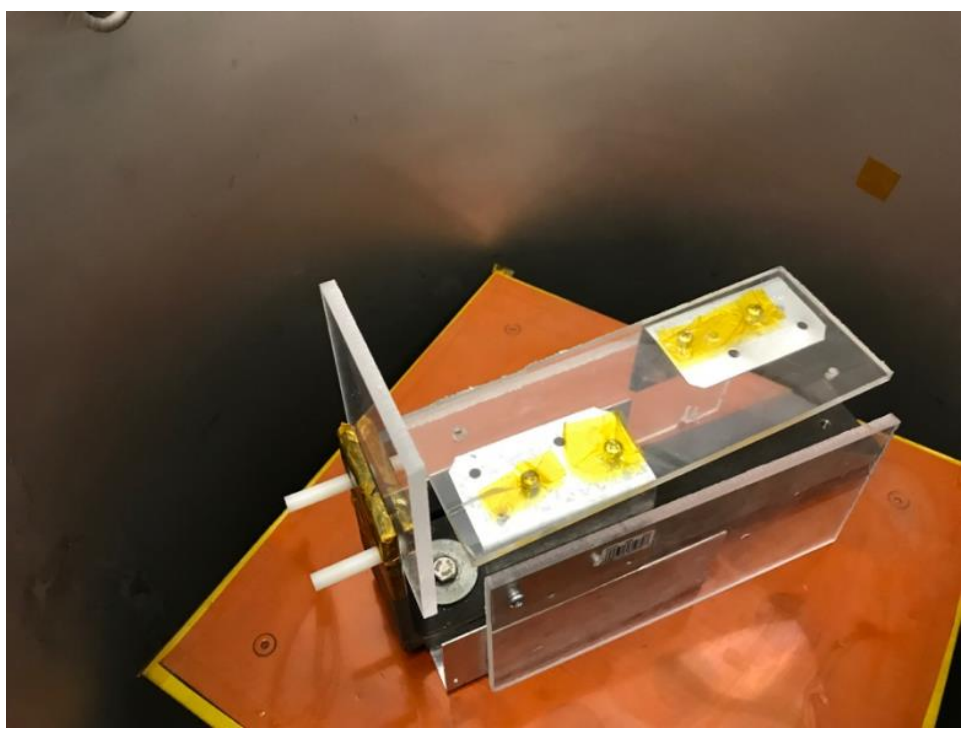

Fig. 4 Stand Set Up

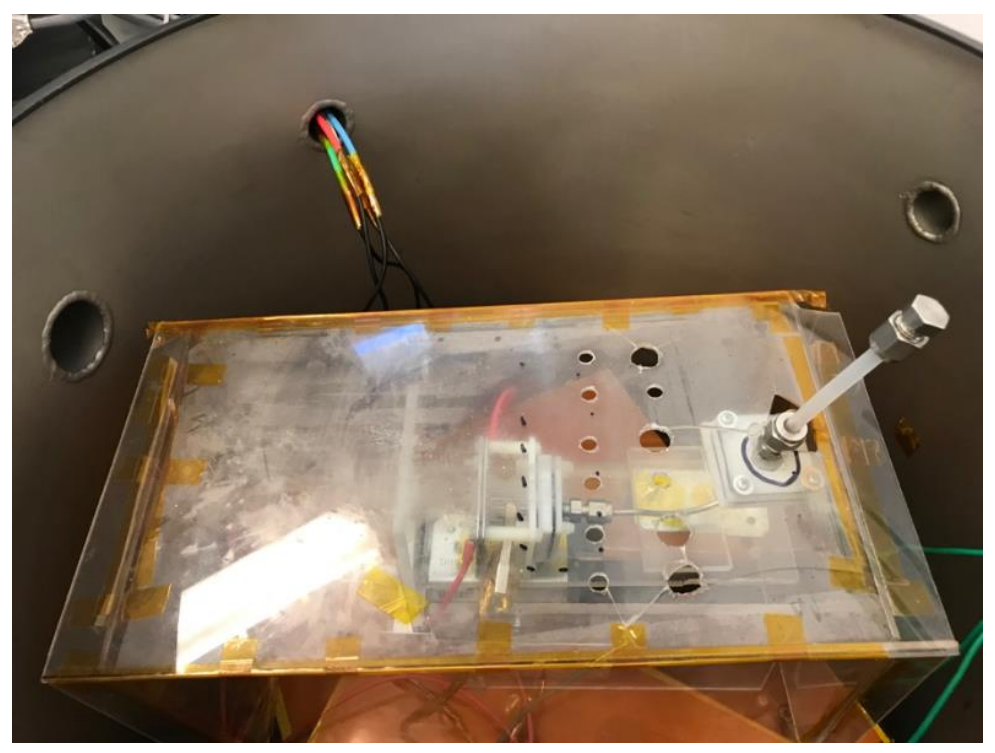

Fig. 5 Complete Set Up 
KEY RESULTS AND DISCUSSION:

1) Estimate colloid thruster performance
a. Estimate thrust produced
b. Estimate thruster efficiency
c. Estimate $I_{\mathrm{sp}}$

2) Discuss the spray pattern on the collection plate. Does it make sense? Why or why not?

3) What can be done to improve thruster efficiency?

4) What additional steps can be taken to improve the testing of the thruster performance?

5) Give some examples of when a colloid thruster can be used in flight.

\section{ASSESSMENT:}

In groups of 3-4 prepare a slide deck outlining your experiment. The following items should be included as a minimum:

- A description of a colloid thruster and how they produce thrust

- An outline of your experimental procedure

- Key results and discussion

\section{MARKING SCHEME:}

Background (Electrospray, thrust production, etc)

(10 points)

Outline of experiment procedure

(10 points)

Key results and thruster performance estimates

(15 points)

Formatting, language, and layout

(5 points) 
Instructor's Note: Here are some additional diagrams to help with the set up.

\section{Chamber Schematic}

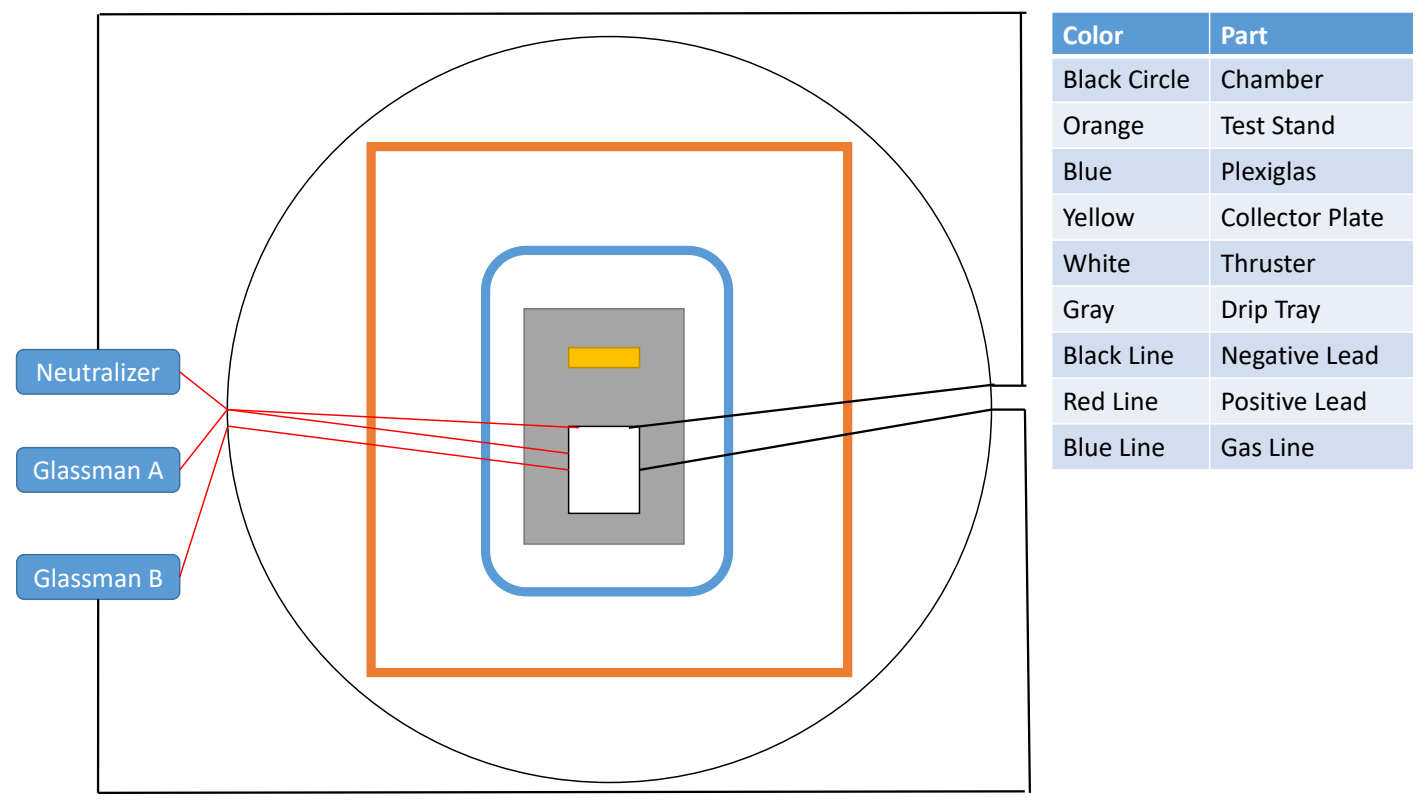

\section{Electrical Schematic}

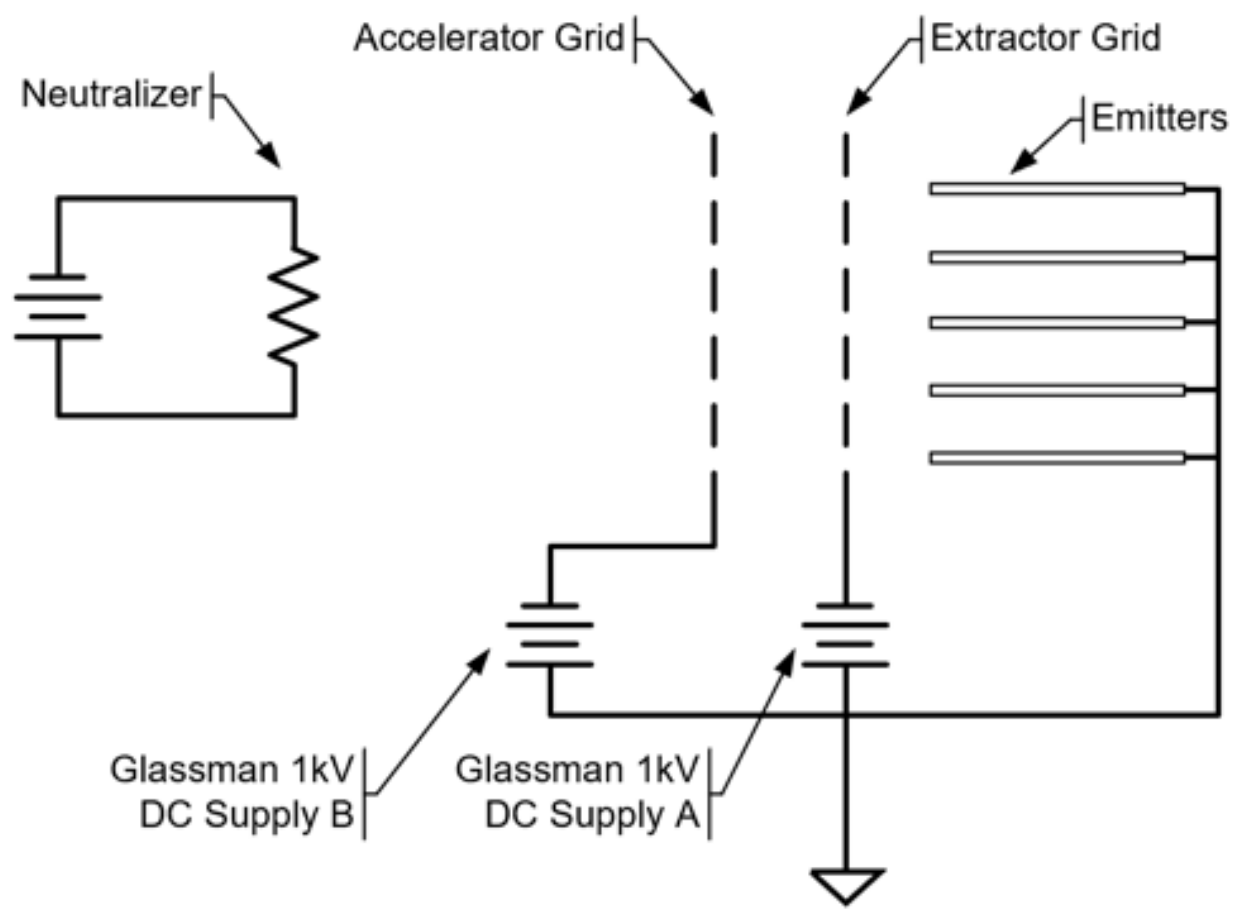




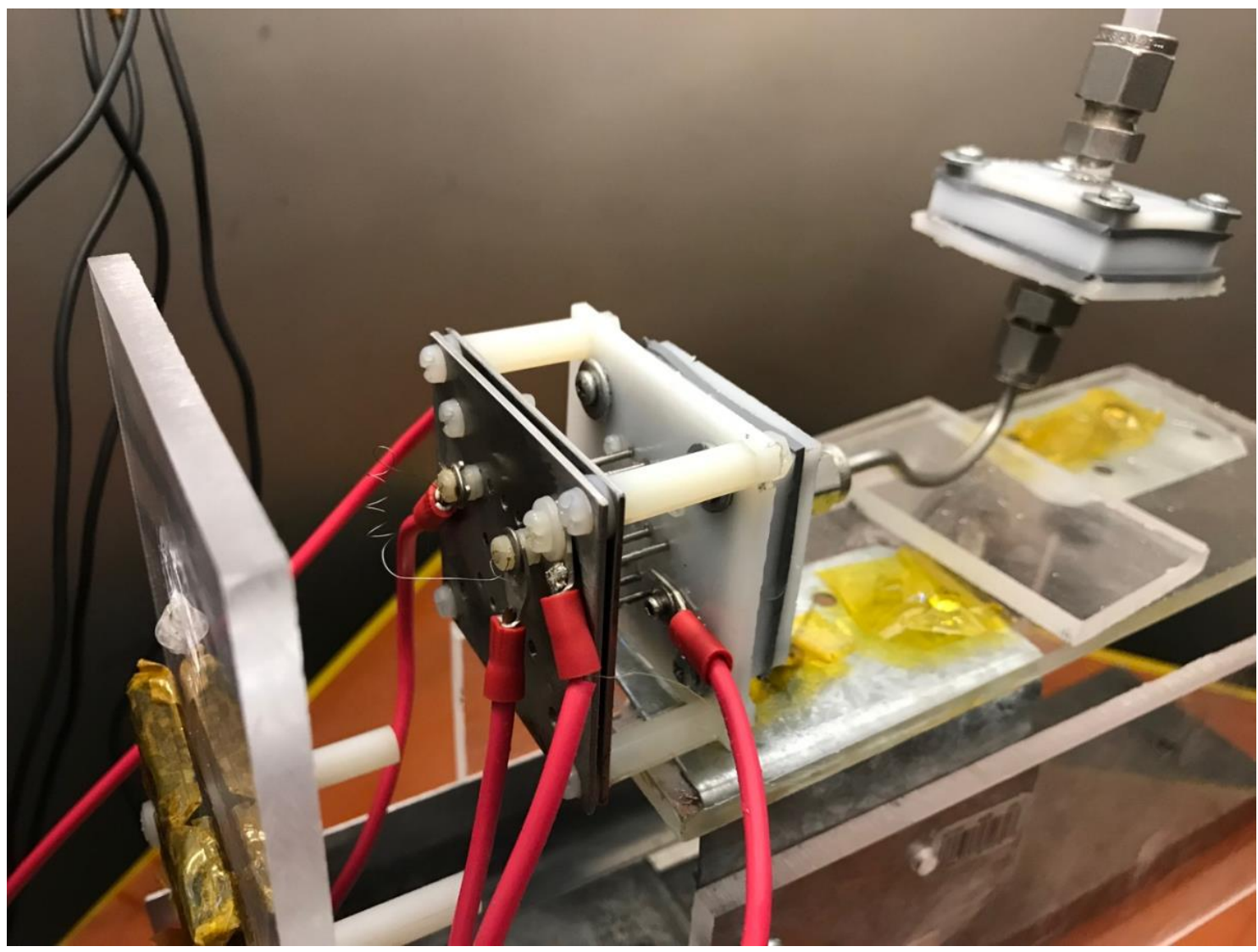

Arcing Troubleshoot Tips:

- If the chamber is near 90 mTorr, try having it pump down to 80 or 70 mTorr.

- If the chamber is $\sim 60 \mathrm{mTorr}$ and still arcing, vent the chamber and add some nylon washers in between the extractor grid and nylon stand offs. This will increase the gap to help with arcing but will lower the thrust produced. Be sure to make note of the new distance in the calculations. 
Appendix D

OPERATING PROCEDURES 


\section{Procedure}

Setting Up the Chamber

1) Locate the Student Vacuum Chambers in the southwest corner of the Space Environments Lab

2) Pick the chamber that is not being utilized for any experimentation

a. If both chambers are empty, the chamber on the right is preferred

3) Ensure that the KF High Voltage electrical feed through and KF gas feed through are connected in the chamber

a. If either or both types of feed throughs are missing, they can be found in the boxes on the cabinets north of the chambers

4) Locate the two Glassman Series FC High Voltage Power Supplies

a. They should be on the rack in between the chambers

b. For simplicity, the top power supply will be Glassman A, and the bottom one will be Glassman B.

5) Locate the HP 6263B DC Power Supply

a. This should be on the rack in between the chambers

6) Connect the power supplies to the electrical feed through and make note of the corresponding connections to the inside of the chamber

Setting Up the Colloid Thruster

1) Inspect the thruster components to ensure all components are in operating order

a. This includes but is not limited to making sure both grids are clean, emitter tips are not bent or broken, all wires are properly soldered to their respective connection points, and filament neutralizer is attached. See Diagram A

2) Assemble the thruster as shown in the exploded diagram

3) Unscrew the back of the propellant reservoir, fill the cavity with glycerol, and reassemble

a. Fill slowly as there is another cavity in the main thruster that is filled

b. Be sure to wear gloves and safety glasses while handling glycerol

c. If skin or eyes come into contact with glycerol, wash skin thoroughly with soap and water and rinse eyes out thoroughly at eye wash station

d. Glycerol is mostly benign and stable, but always error on the side of caution as some people may have negative reactions to glycerol

e. See Safety Data Sheet for reference

4) Place the Plexiglas stand over the drip tray and place the thruster on the Plexiglas block

Integrating the Thruster into the Chamber

1) Insert the test stand into the chamber 
2) Place the drip tray with the thruster and Plexiglas test stand onto the test stand

3) Place the collector place on the test stand approximately $2.5 \mathrm{~cm}$ away from the accelerator grid of the thruster

4) Connect the leads from the extractor plate and emitters to the leads on the electrical feed through to Glassman A

5) Connect the leads from the accelerator grid to the electrical feed through to Glassman B

6) Connect the HP Power Supply leads to the filament neutralizer

7) Check continuity and make sure the chamber is electrically isolated from the power supplies

8) Obtain the mass of the collector plate and attach to the stand

a. At this point, the set up should look like Photo 2

9) Place the Plexiglas box (See Photo 3) on top of the entire system

Running the Experiment

1) Place the lid on top of the chamber and tighten it down

2) Pump the chamber down as low as it can go

a. The chamber must be lower than 90 mTorr to avoid voltage breakdown

3) Turn on the power supplies and set them to the desired voltages

a. Glassman A should be set to $1000 \mathrm{~V}$

b. Glassman B can be set between 700-1000 V

c. HP Power Supply should be set to $3 \mathrm{~V}$ and $1.5 \mathrm{~A}$

4) Run the thruster for 5-7 minutes

5) Turn off power supplies

6) Vent the chamber

7) Removed the lid, Plexiglas box, and Plexiglas collector plate

a. Take the mass the Plexiglas collector plate to see how much propellant was emitted

Clean Up

1) Remove all components from the chamber

2) Put the lid back on top of the chamber

3) Clean and return the Plexiglas box, Plexiglas collector plate, drip tray, and Plexiglas test stand

4) Empty out the main thruster component and propellant reservoir of any excess propellant

a. Discard any unused glycerol in the sink

b. Disassemble the thruster and clean off as much glycerol as possible with alcohol

5) Disconnect the power supplies from the chamber

Schematics 
Electrical Schematic

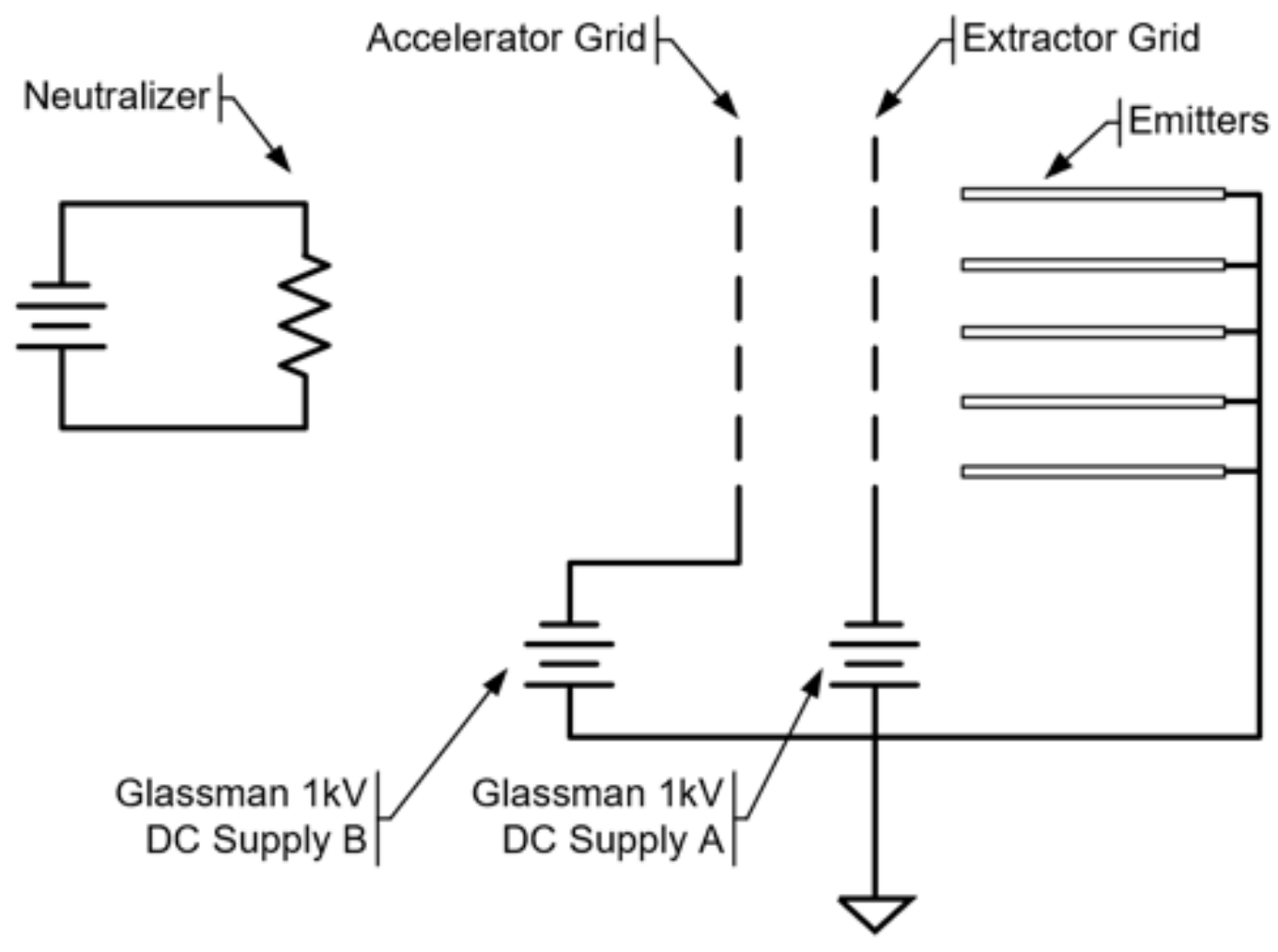

Chamber Schematic

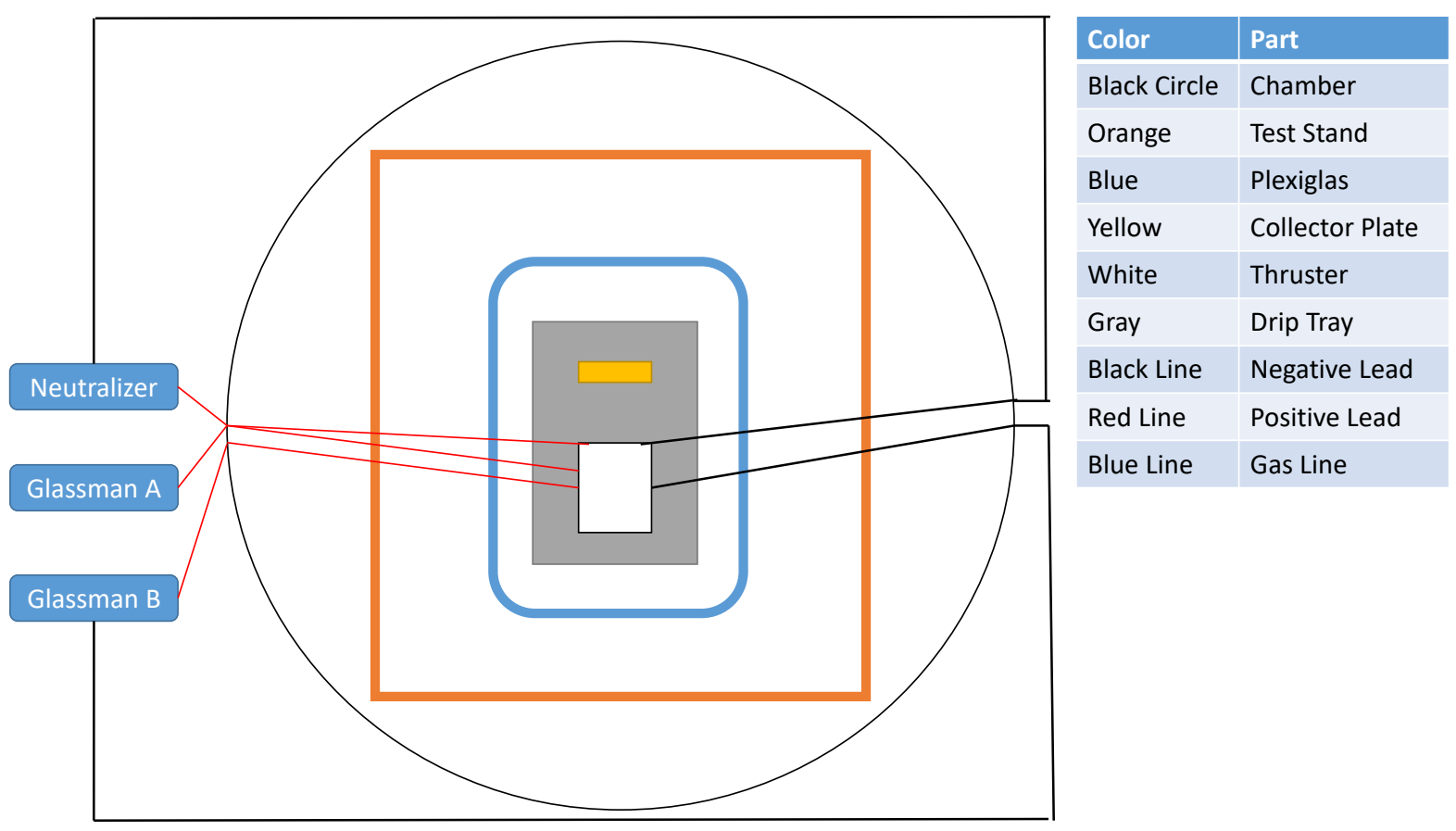




\section{Exploded View}

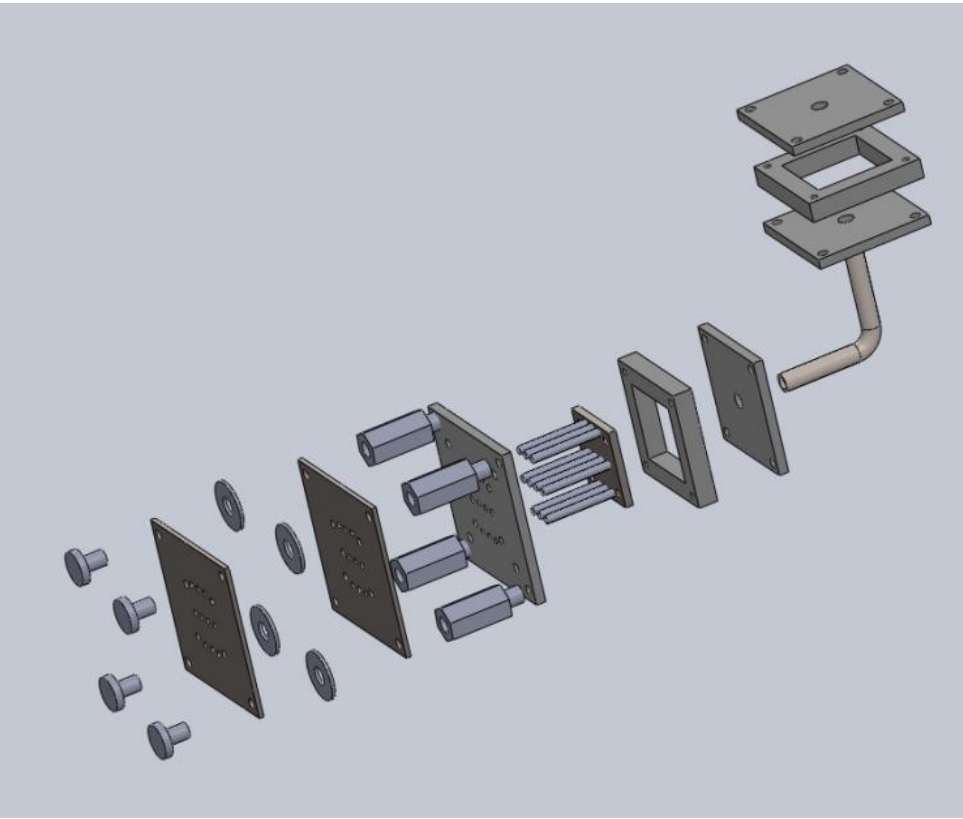

\section{Photos}

Photo 1

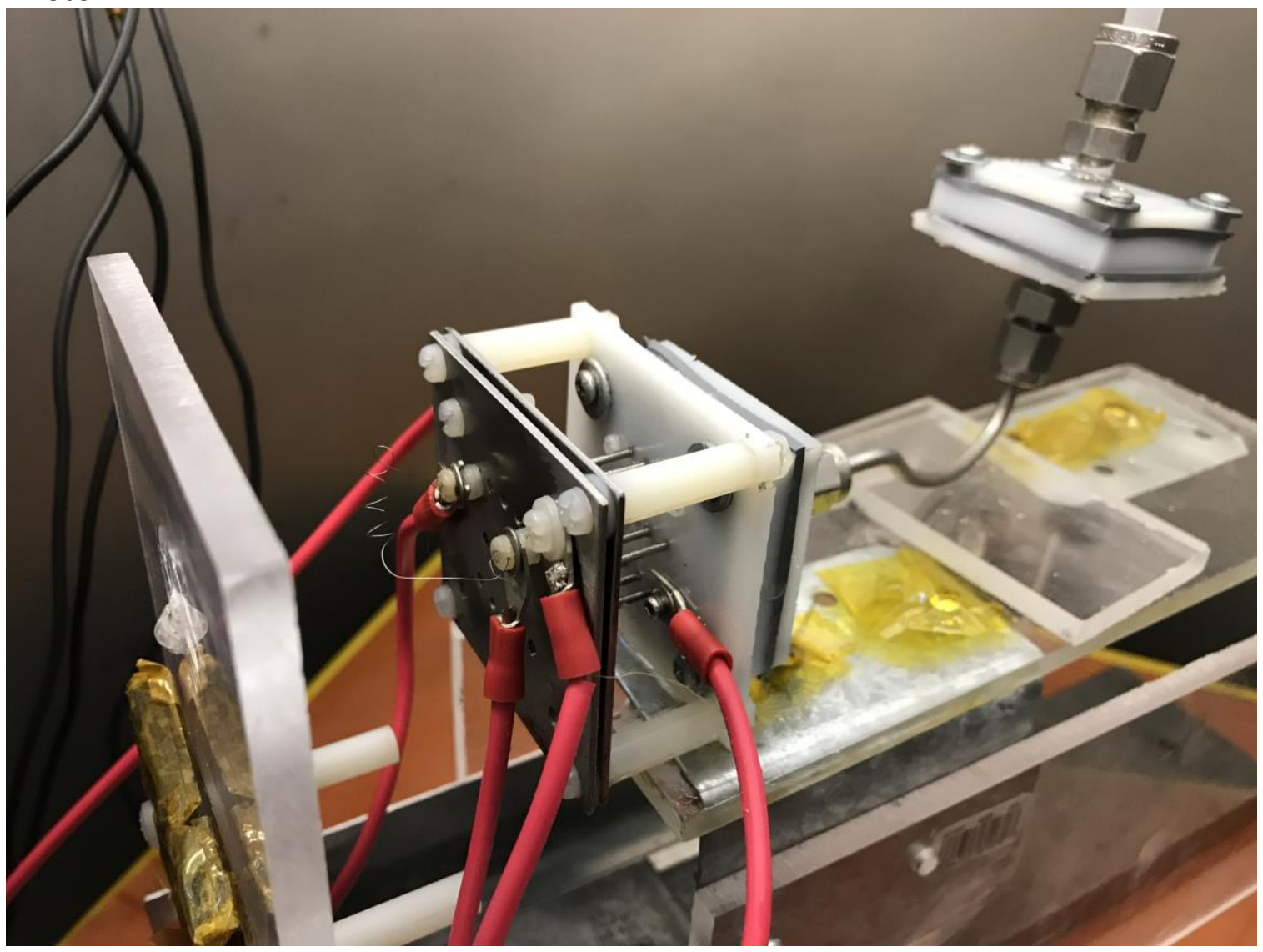


Photo 2

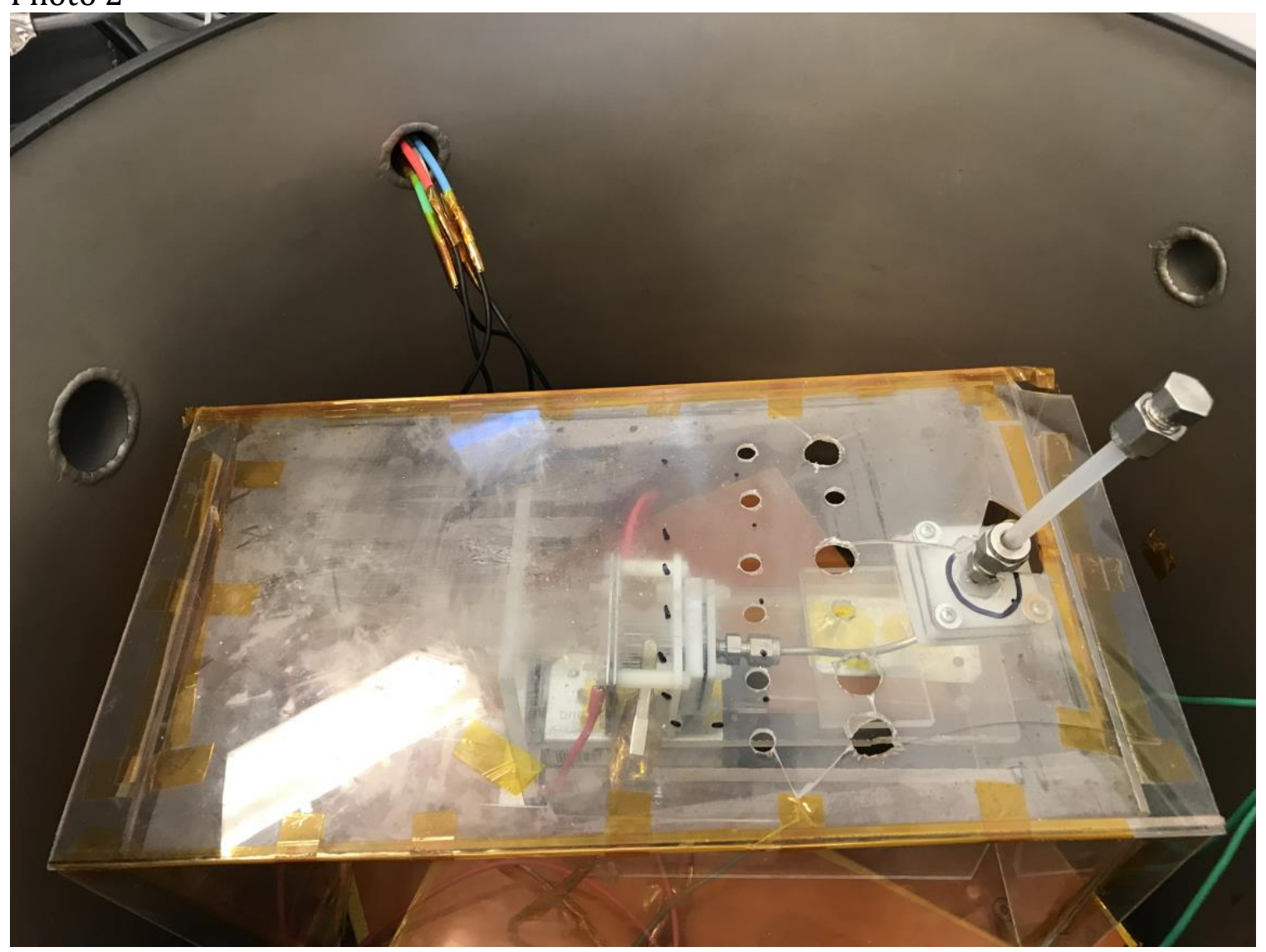




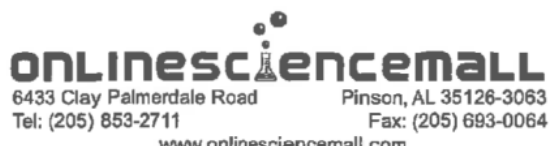

CHEMTREC 24 Hour Emergency

Phone Number (800) 424-9300 For laboratory use only.

Not for drug, food or household use.

\begin{tabular}{l|l}
\hline Product & GLYCERIN \\
\hline Synonyms & Glycerol \\
Section 2 & Hazards Identification
\end{tabular}

This substance or mixture has not been classified as hazardous according to the Globally Harmonized System (GHS) of Classification and Labeling of Chemicals.

Supplemental information:

Do not breathe mist/vapours/spray. Do not get in eyes, on skin, or on clothing. Wear protective gloves/protective clothing/eye protection/face protection. Wash hands thoroughly after handling. Get medical attention if you feel unwell.

Signal word: None required

Pictograms: No symbol required

Target organs: None known

GHS Classification: None required

GHS Label information: Hazard statement: None required

Precautionary statement: None required

Ca Prop 65: This product does not contain any chemicals known to the State of California to cause cancer or reproductive toxicity.

$\begin{array}{lcccc}\text { Section } 3 & \text { Composition I Information on Ingredients } & & \\ \text { Chemical Name } & \text { CAS \# } & \% & \text { EINECS } \\ \text { Glycerin } & 56-81-5 & 100 \% & 200-289-5\end{array}$

Section 4

First Aid Measures

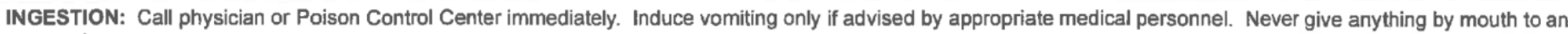
unconscious person.

INHALATION: Remove to fresh air. If not breathing, give artificial respiration. If breathing is difficult, give oxygen. Get medical attention.

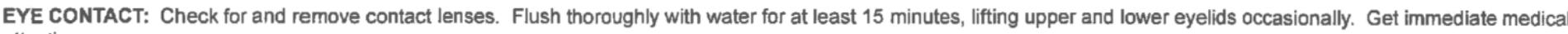
attention.

SKIN ABSORPTION: Remove contaminated clothing. Flush thoroughly with mild soap and water. If irritation occurs, get medical attention.

\section{Section $5 \quad$ Fire Fighting Measures}

Suitable Extinguishing Media: DO NOT USE WATER. Carbon dioxide, dry chemical, dry sand, alcohol foam.

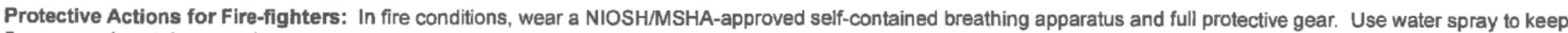
fire-exposed containers cool.

Specific Hazards: During a fire, irritating and highly toxic gases may be generated by thermal decomposition or combustion.

Personal Precautions: Evacuate personnel to safe area. Use proper personal protective equipment as indicated in Section 8 . Provide adequate ventilation.

Environmental Precautions: Avoid runoff into storm sewers and ditches which lead to waterways.

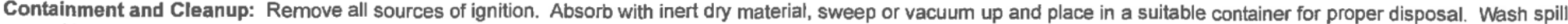
area with soap and water. 
Precautions for Safe Handling: Read label on container before using. Do not wear contact lenses when working with chemicals. Keep out of reach of children. Avoid contact with eyes, skin and clothing. Do not inhale vapors, spray or mist. Use with adequate ventilation. Avoid ingestion. Wash thoroughly after handling. Remove and wash clothing before reuse.

Conditions for Safe Storage: Store in a cool, well-ventilated area away from incompatible substances. Keep away from ignition sources.

\section{Section $8 \quad$ Exposure Controls / Personal Protection}

\section{Exposure Limits: Chemical Name}

Glycerin

$\begin{array}{ccc}\text { ACGIH (TLV) } & \text { OSHA (PEL) } & \text { NIOSH (REL) } \\ \text { TWA: } 10 \mathrm{mg} / \mathrm{m}^{3} \text { (mist) } & \text { TWA: } 5 \mathrm{mg} / \mathrm{m}^{3} \text { (respirable fraction) } & \text { None established }\end{array}$

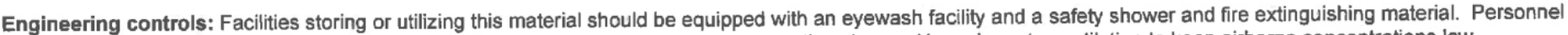
should wear safety glasses, goggles, or faceshield, lab coat or apron, appropriate protective gloves. Use adequate ventilation to keep airborne concentrations low.

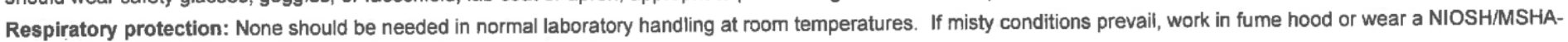
approved respirator.

\section{Section $9 \quad$ Physical \& Chemlcal Properties}

Appearance: Clear, colorless liquid.

Odor: No odor.

Odor threshold: Data not available.

pH: Approximately 7.0

Melting / Freezing point: $18^{\circ} \mathrm{C}\left(64^{\circ} \mathrm{F}\right)$

Boiling point: $290^{\circ} \mathrm{C}\left(554^{\circ} \mathrm{F}\right)$

Flash point: $177^{\circ} \mathrm{C}\left(351^{\circ} \mathrm{F}\right) \mathrm{COC}$

\section{Section 10}

Stability \& Reactivity

Evaporation rate ( Ether $=1$ ): $>1$

Flammability (solid/gas): Data not available.

Explosion limits: Lower / Upper: Data not available

Vapor pressure $(\mathrm{mm} \mathrm{Hg}): 0.01 \mathrm{mbar} @ 20^{\circ} \mathrm{C}$

Vapor density (Air = 1): Data not available

Relative density (Specific gravity): $1.2607\left(25 / 25^{\circ} \mathrm{C}\right)$

Solubility(ies): Complete

\section{Chemical stability: Stable Hazardous polymerization: Will not occur.}

Conditions to avoid: Excessive temperatures and heat. Absorbs moisture from the air.

Incompatible materials: Strong oxidizers.

Hazardous decomposition products: Poisonous Acrolein fumes.

\section{Section $11 \quad$ Toxicological Information}

Acute toxicity: Oral-rat LD50: $25,000 \mathrm{mg} / \mathrm{kg}$

Skin corrosion/irritation: Dermal-rabbit - $18,700 \mathrm{mg} / \mathrm{kg}$

Serious eye damage/irritation: Rabbit - practically non-irritating

Respiratory or skin sensitization: Data not available

Germ cell mutagenicity: Data not available

Carcinogenity: Data not available

NTP: No component of this product present at levels greater than or equal to $0.1 \%$ is identified as a known or anticipated carcinogen by NTP.

IARC: No component of this product present at levels greater than or equal to $0.1 \%$ is identified as probable, possible or confirmed human carcinogen by IARC.

OSHA: No component of this product present at levels greater than or equal to $0.1 \%$ is identified as a carcinogen or potential carcinogen by OSHA.

Reproductive toxicity: Data not available

STOT-single exposure: Data not available

STOT-repeated exposure: Data not available

Aspiration hazard: Data not available

Potential health effects:

Inhalation: Inhalation of mist are irritating to the respiratory tract.

Ingestion: Ingestion is considered to be a very low hazard.

Skin: Skin contact shows no significant irritation. Not absorbed through the skin.

Eyes: May cause slight eye irritation.

Signs and symptoms of exposure: See Potential health effects above.

Additional information: RTECS \#: MA8050000

\section{Section $12 \quad$ Ecological Information}

Toxicity to fish: Low toxicity to aquatic organisms. LC50 (96 hour) fish $>5,000 \mathrm{mg} / \mathrm{l}$

Toxicity to daphnia and other aquatic invertebrates: EC50 (24 hour) Daphnia magna >10,000 mg/

Toxicity to algae: No data available

Persistence and degradability: No data available Bioaccumulative potential: No data available

Mobility in soil: No data available $\quad$ PBT and vPvB assessment: No data available

Other adverse effects: An environmental hazard cannot be excluded in the event of unprofessional handling or disposal.

\section{Section 13 Disposal Considerations}

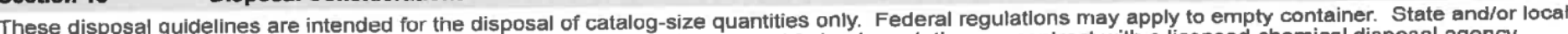

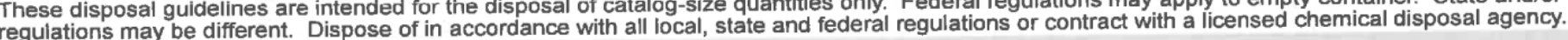

Section $14 \quad$ Transport Information (US DOT / CANADA TDG)

UNINA number: Not applicable Shipping name: Not Regulated

Hazard class: Not applicable Packing group: Not applicable

Exceptions: Not applicable 2012 ERG Guide \# Not applicable

Reportable Quantity: No Marine pollutant: No

\section{Section 15 Regulatory Information}

A chemical is considered to be listed if the CAS number for the anhydrous form is on the Inventory list. Component TSCA CERLCA (RQ)

Glycerin

Listed Not listed

$\begin{array}{ccc}\text { RCRA code } & \text { DSL } & \text { NDSL } \\ \text { Not listed } & \text { Listed } & \text { Not listed }\end{array}$

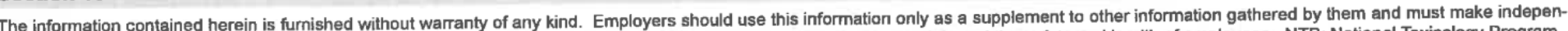

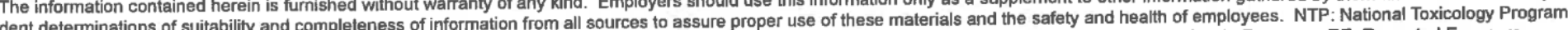

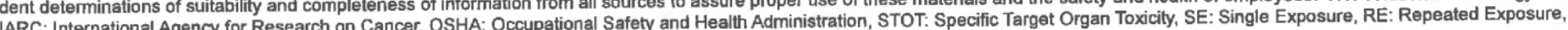

IARC: International Agency for Research

Form 06/2015

Revision Date: May 12, 2016 